\title{
LAMN PROPERTY FOR THE DRIFT AND VOLATILITY PARAMETERS OF A SDE DRIVEN BY A STABLE LÉVY PROCESS
}

\author{
Emmanuelle Clément ${ }^{1, *}$, Arnaud Gloter ${ }^{2}$ and Huong NGuyen ${ }^{3}$
}

\begin{abstract}
This work focuses on the local asymptotic mixed normality (LAMN) property from high frequency observations, of a continuous time process solution of a stochastic differential equation driven by a truncated $\alpha$-stable process with index $\alpha \in(0,2)$. The process is observed on the fixed time interval $[0,1]$ and the parameters appear in both the drift coefficient and scale coefficient. This extends the results of Clément and Gloter [Stoch. Process. Appl. 125 (2015) 2316-2352] where the index $\alpha \in(1,2)$ and the parameter appears only in the drift coefficient. We compute the asymptotic Fisher information and find that the rate in the LAMN property depends on the behavior of the Lévy measure near zero. The proof relies on the small time asymptotic behavior of the transition density of the process obtained in Clément et al. [Preprint HAL-01410989v2 (2017)].
\end{abstract}

Mathematics Subject Classification. 60G51, 62F12, 60H07, 60F05, 60G52, 60J75.

Received February 27, 2017. Accepted January 24, 2018.

\section{INTRODUCTION}

Modeling with pure jump Lévy processes plays an important role in many fields, especially in mathematical finance (see for example $[3,16,20]$ ) and parametric inference for such processes is a currently active subject.

In this paper, we are concerned with parametric estimation of a stochastic differential equation driven by a pure jump Lévy process, from high-frequency observations on a fixed observation time. More precisely we consider the statistical experiment $\left(\mathbb{R}^{n}, \mathcal{B}_{n},\left(\mathbb{P}_{n}^{\beta}\right)_{\beta \in \Theta \subset \mathbb{R}^{2}}\right)$ corresponding to the observation of a Lévy driven stochastic equation at discrete times $\left(X_{\frac{i}{n}}^{\beta}\right)_{1 \leq i \leq n}$, solution of

$$
X_{t}^{\beta}=x_{0}+\int_{0}^{t} b\left(X_{s}^{\beta}, \theta\right) \mathrm{d} s+\sigma L_{t}, \quad t \in[0,1],
$$

where $\left(L_{t}\right)_{t \in[0,1]}$ is a truncated $\alpha$-stable process with exponent $\alpha \in(0,2)$ and $\beta=(\theta, \sigma)^{T} \in \mathbb{R} \times(0, \infty)$ is an unknown parameter to be estimated. We prove in this work that the local asymptotic mixed normality property

Keywords and phrases: Lévy process, stable process, Malliavin calculus for jump processes, LAMN property, parametric estimation.

${ }^{1}$ Mathématiques et Informatique pour la Complexité, Fédération de Mathématiques FR 3487, CentraleSupélec, Université Paris-Saclay, 91190 Gif-sur-Yvette, France.

${ }^{2}$ Université d'Évry Val d'Essonne, Laboratoire de Mathématiques et Modélisation d'Evry, UMR 8071, 23 Boulevard de France, 91037 Évry Cedex, France.

3 Université Paris-Est, LAMA UMR 8050, UPEMLV, CNRS, UPEC, 77454 Marne-la-Vallée, France.

* Corresponding author: emmanuelle.clement@u-pem.fr 
(LAMN) holds for the parameter $\beta$. The LAMN property has been introduced by Jeganathan $[14,15]$ to extend to the markovian case the LAN property introduced in the pioneer works by Lecam and Hájek (see [10, 21]) in the i.i.d. case. This property permits in particular to identify the optimal estimation rate for the parameters $\theta$ and $\sigma$ and the asymptotic Fisher information.

Parametric inference and LAN property for pure jump Lévy processes based on high frequency observations have been investigated in several papers, see for example Aït-Sahalia and Jacod [1, 2], Kawai and Masuda $[17,18]$, Masuda [22]. In particular, in [22], the LAN property is established and estimators are proposed for the parameters $(\theta, \sigma, \alpha)$ in the model $X_{t}=\theta t+\sigma L_{t}^{\alpha}$, where $\left(L_{t}^{\alpha}\right)$ is an $\alpha$-stable process. Aït-Sahalia and Jacod $[1,2]$ considered the model $X_{t}=\sigma L_{t}^{\alpha}+\theta Y_{t}$ where $\left(Y_{t}\right)$ is a Lévy process, independent of $\left(L_{t}^{\alpha}\right)$ and dominated by $\left(L_{t}^{\alpha}\right)$. More recently, Ivanenko, Kulik and Masuda [12] proved the LAN property for the parameter $(\theta, \sigma)$ in the model $X_{t}=\theta t+\sigma Z_{t}+U_{t}$, where $Z$ is a locally $\alpha$-stable process and $U$ is an independent and less active process. In all these works, the increments $\left(X_{\frac{i}{n}}-X_{\frac{i-1}{n}}\right)_{1 \leq i \leq n}$ are independent and the transition density of the discrete time process $\left(X_{\frac{i}{n}}\right)_{1 \leq i \leq n}$ is almost explicit. Extensions to stochastic equations driven by pure jump Lévy processes are not immediate and require a different approach since the transition density of the Markov chain $\left(X_{\frac{i}{n}}\right)_{1 \leq i \leq n}$ is unknown. Moreover they involve a random asymptotic Fisher information and lead to the LAMN property. Concerning the parametric estimation of a stochastic differential equation driven by a pure jump Lévy process from high frequency observations on a fixed observation time, we can mention the recent paper by Masuda [23] where some estimators of the parameters $(\theta, \sigma)$ are proposed for the general equation

$$
X_{t}=x_{0}+\int_{0}^{t} b\left(X_{s}, \theta\right) \mathrm{d} s+\int_{0}^{t} c\left(X_{s-}, \sigma\right) \mathrm{d} L_{s}
$$

where $L$ is a locally $\alpha$-stable process, with $\alpha \in[1,2)$. However in that case the asymptotic efficiency of the estimators is not yet establish and to our knowledge, the only result in that direction is given in Clément and Gloter [5], where the LAMN property is proved for the estimation of the drift parameter $\theta$ for the process solution of (2.1) (with $\sigma=1$ ), in the case $\alpha \in(1,2)$. They show that the LAMN property is satisfied with rate $r_{n}=n^{\frac{1}{2}-\frac{1}{\alpha}}$ and information $\mathcal{I}_{\theta}=\int_{0}^{1} \partial_{\theta} b\left(X_{s}^{\beta}, \theta\right)^{2} \mathrm{~d} s \int_{\mathbb{R}} \frac{\varphi_{\alpha}^{\prime}(u)^{2}}{\varphi_{\alpha}(u)} \mathrm{d} u$, where $\varphi_{\alpha}$ is the density of the standard $\alpha$-stable distribution with characteristic function $u \mapsto e^{-C(\alpha)|u|^{\alpha}}$.

Based on the main ideas of [5] and using the results of [6], we extend in the present paper these results to $\alpha \in(0,2)$ and prove that the LAMN property holds for the parameters $(\theta, \sigma)$ with rate $r_{n}=\left(\begin{array}{cc}n^{\frac{1}{2}-\frac{1}{\alpha}} & 0 \\ 0 & n^{-\frac{1}{2}}\end{array}\right)$ and information matrix $\mathcal{I}=\left(\begin{array}{cc}\mathcal{I}_{11} & 0 \\ 0 & \mathcal{I}_{22}\end{array}\right)$ where $\mathcal{I}_{11}=\frac{1}{\sigma^{2}} \mathcal{I}_{\theta}$ and $\mathcal{I}_{22}=\frac{1}{\sigma^{2}} \int_{\mathbb{R}} \frac{\left(\varphi_{\alpha}(u)+u \varphi_{\alpha}^{\prime}(u)\right)^{2}}{\varphi_{\alpha}(u)} \mathrm{d} u$. The proof is mainly based on the $L^{2}$-regularity property of the transition density (see Jeganathan [14]) and on Malliavin calculus (see for example Gobet [8] for the use of Malliavin calculus in the case of a diffusion process). The $L^{2}$ regularity property is established here by using the asymptotic behavior of the density of the process solution of (2.1) in small time as well as its derivative with respect to the parameter, given in [6] and based on the Malliavin calculus for jump processes developed by Bichteler et al. [4]. It also requires a careful study of the asymptotic behavior of the information matrix based on one observation of the process, this is the subject of Section 3. This paper contains also an independent and interesting result stating a continuity property with respect to the conditioning variable in a conditional expectation (see Prop. 6.8).

This paper is organized as follows. The main results (asymptotic expansion of the log-likelihood function and LAMN property) are stated in Section 2. Section 3 studies the asymptotic behavior of the Fisher information matrix based on the observation of $X_{\frac{1}{n}}^{\beta}$ (as $n$ goes to infinity). The proof of the main results are given in Section 4 and Section 5. Finally, Section 6 contains some additional technical proof required to establish the results of Section 3. 


\section{MAIN RESULTS}

We consider the process $\left(X_{t}^{\beta}\right)_{t \in[0,1]}$ solution to

$$
X_{t}^{\beta}=x_{0}+\int_{0}^{t} b\left(X_{s}^{\beta}, \theta\right) \mathrm{d} s+\sigma L_{t} \quad t \in[0,1]
$$

where $\left(L_{t}\right)_{t \in[0,1]}$ is a pure jump Lévy process defined on some probability space $(\Omega, \mathcal{A}, \mathcal{P})$ and we are interested in the statistical properties of the process $\left(X_{t}^{\beta}\right)$, based on the discrete time observations $\left(X_{i / n}^{\beta}\right)_{i=0, \ldots n}$. We assume that the following assumptions are fulfilled.

$\mathbf{H}_{1}$ :

(a) The function $b$ has bounded derivatives up to order five with respect to both variables.

$\left(b_{i}\right)$ The Lévy process $\left(L_{t}\right)_{t \in[0,1]}$ is given by $L_{t}=\int_{0}^{t} \int_{[-1,1]} z\{\bar{\mu}(d s, d z)-\bar{v}(\mathrm{~d} s, \mathrm{~d} z)\}+\int_{0}^{t} \int_{[-1,1]^{c}} z \bar{\mu}(\mathrm{d} s, \mathrm{~d} z)$ where $\bar{\mu}$ is a Poisson random measure, with compensator $\bar{v}(\mathrm{~d} t, \mathrm{~d} z)=\mathrm{d} t \times F(z) \mathrm{d} z$ where $F(z)$ is given on $\mathbb{R}$ by $F(z)=\frac{1}{|z|^{\alpha+1}} 1_{|z| \neq 0} \tau(z), \alpha \in(0,2)$. We assume that $\tau$ is a non negative smooth function equal to 1 on $[-1,1]$, vanishing on $[-2,2]^{c}$ such that $0 \leq \tau \leq 1$.

$\left(b_{i i}\right)$ We assume that $\forall p \geq 1, \int_{\mathbb{R}}\left|\frac{\tau^{\prime}(u)}{\tau(u)}\right|^{p} \tau(u) \mathrm{d} u<\infty, \int_{\mathbb{R}}\left|\frac{\tau^{\prime \prime}(u)}{\tau(u)}\right|^{p} \tau(u) \mathrm{d} u<\infty$.

These assumptions are sufficient to ensure that (2.1) has an unique solution belonging to $\mathbf{L}^{p}, \forall p \geq 1$, and that $X_{t}^{\beta}$ admits a density, for $t>0$ (see [24]). Moreover, it is proved in [6] that this density is differentiable with respect to $\beta$.

Remark 2.1. Our assumptions on the Lévy measure $F$ are quite restrictive and reduce the generality of our results but simplify the proofs which nevertheless remain still technical. There are mainly two important properties required on the Lévy measure in our approach. First, since our method is based on Malliavin calculus, the $L^{p}$-bounds for the tails of the Lévy process are crucial to ensure that our process belongs to the Malliavin space. Secondly, the stable behavior of the Lévy measure around zero is also required to make the rescaled process $\left(n^{1 / \alpha} L_{t / n}\right)$ close to the $\alpha$-stable process $\left(L_{t}^{\alpha}\right)$. The introduction of the truncation function $\tau$ permits to address both issues and to avoid more technical proofs. In particular it permits to ensure that the process $\left(n^{1 / \alpha} L_{t / n}\right)$ has no jump of size larger than $2 n^{1 / \alpha}$ and consequently makes easier the control of the asymptotic behavior of the Malliavin weights (mainly studied in [6]). Moreover the exact stable behavior of the Lévy measure around zero $(\tau=1)$ gives the equality between the rescaled process $\left(n^{1 / \alpha} L_{t / n}\right)$ and the $\alpha$-stable process $\left(L_{t}^{\alpha}\right)$, and also the equality of the corresponding Poisson measures, on a set $A_{n}$ whose complementary has small probability $\left(\mathbb{P}\left(A_{n}^{c}\right) \leq C / n\right.$, see Lem. 3.3). This property is repeatedly used in our proofs (see for example the proof of Thm. 2.5) and is also essential to study the limit of the Malliavin weights in [6].

However, since the information matrix obtained in the LAMN property (established in Cor. 2.6) does not depend on the function $\tau$, this suggests that the same result probably holds for a more general Lévy measure even with no integrability conditions on the large jumps and that the truncation or integrability assumptions should only be introduced in the proof sections. To that end, a possible extension of our paper (and also of [6]) could be to replace $\tau$ by a more general function $g$ such that $g(0)=1$ and satisfying $\left(b_{i i}\right)$, but up to now we do not know how to obtain the key results established in [6] in this more general context.

Before stating our main results, we introduce some notations which are used throughout this paper. For a vector $h \in \mathbb{R}^{2}, h^{T}$ denotes the transpose of $h$, and $|h|$ denotes the euclidean norm. For a function $f$ defined on $\mathbb{R} \times \mathbb{R}^{2}$ depending on both variables $(x, \beta)$, here $\beta=(\theta, \sigma)^{T} \in \mathbb{R} \times(0,+\infty)$, we denote by $f^{\prime}$ the derivative of $f$ with respect to the variable $x$, by $\partial_{\theta} f$ the derivative of $f$ with respect to the parameter $\theta$, by $\partial_{\sigma} f$ the derivative of $f$ with respect to the parameter $\sigma$, and $\nabla_{\beta} f=\left(\begin{array}{c}\partial_{\theta} f \\ \partial_{\sigma} f\end{array}\right)$. 
We denote by $p_{i / n}^{\beta}(x, y)$ the transition density of the homogeneous Markov chain $\left(X_{i / n}^{\beta}\right)_{i=0, \ldots n}$, by $\left(\mathcal{F}_{i / n}\right)_{i}$ the $\sigma$-field such that $\mathcal{F}_{i / n}=\sigma\left(X_{s}^{\beta}, s \leq i / n\right)=\sigma\left(L_{s}, s \leq i / n\right)$ and by $\mathbb{P}_{n}^{\beta}$ the law of the vector $\left(X_{\frac{1}{n}}^{\beta}, \ldots, X_{1}^{\beta}\right)$ on $\mathbb{R}^{n}$.

Our first result is an asymptotic expansion of the log-likelihood ratio.

Theorem 2.2. We assume that $\boldsymbol{H}_{1}$ holds. Let $r_{n}=\left(\begin{array}{cc}n^{\frac{1}{2}-\frac{1}{\alpha}} & 0 \\ 0 & n^{-\frac{1}{2}}\end{array}\right)$, then for every $h \in \mathbb{R}^{2}$

$$
\log \frac{d \mathbb{P}_{n}^{\beta+r_{n} h}}{d \mathbb{P}_{n}^{\beta}}\left(X_{\frac{1}{n}}^{\beta}, \ldots, X_{1}^{\beta}\right)=h^{T} J_{n}(\beta)^{\frac{1}{2}} N_{n}(\beta)-\frac{1}{2} h^{T} J_{n}(\beta) h+o_{\mathcal{P}}(1),
$$

with

$$
\begin{aligned}
& J_{n}(\beta)=r_{n} \sum_{i=0}^{n-1} \mathbb{E}\left[\xi_{i, n}(\beta) \xi_{i, n}^{T}(\beta) \mid \mathcal{F}_{i / n}\right] r_{n} \\
& N_{n}(\beta)=J_{n}(\beta)^{\frac{-1}{2}} r_{n} \sum_{i=0}^{n-1} \xi_{i, n}(\beta), \\
& \xi_{i, n}(\beta)=\left(\begin{array}{c}
\left.\frac{\partial_{\theta} p_{\frac{1}{n}}^{\beta}}{p_{\frac{1}{n}}^{\beta}}\left(X_{\frac{i}{n}}^{\beta}, X_{\frac{i+1}{n}}^{\beta}\right)\right) \\
\frac{\partial_{\sigma} p_{\frac{1}{n}}^{\beta}}{p_{\frac{1}{n}}^{\beta}}\left(X_{\frac{i}{n}}^{\beta}, X_{\frac{i+1}{n}}^{\beta}\right)
\end{array}\right)
\end{aligned}
$$

We can precise the asymptotic behavior of $J_{n}(\beta)$ and $N_{n}(\beta)$. Let $\varphi_{\alpha}$ be the density of $L_{1}^{\alpha}$, where $\left(L_{1}^{\alpha}\right)$ is a centered $\alpha$-stable Lévy process whose Lévy measure is $\frac{\mathrm{d} z}{|z|^{1+\alpha}} 1_{|z| \neq 0}$. We define the following quantity which will be the random asymptotic information of the statistical model:

$$
\mathcal{I}(\beta)=\left(\begin{array}{cc}
\mathcal{I}_{11} & 0 \\
0 & \mathcal{I}_{22}
\end{array}\right)
$$

where

$$
\mathcal{I}_{11}=\frac{1}{\sigma^{2}} \int_{0}^{1} \partial_{\theta} b\left(X_{s}^{\beta}, \theta\right)^{2} \mathrm{~d} s \times \int_{\mathbb{R}} \frac{\varphi_{\alpha}^{\prime}(u)^{2}}{\varphi_{\alpha}(u)} \mathrm{d} u, \quad \mathcal{I}_{22}=\frac{1}{\sigma^{2}} \times \int_{\mathbb{R}} \frac{\left(\varphi_{\alpha}(u)+u \varphi_{\alpha}^{\prime}(u)\right)^{2}}{\varphi_{\alpha}(u)} \mathrm{d} u .
$$

\section{Remark 2.3.}

i) From [2, 12], we know that the parameter $\theta$ of the process $X_{t}^{\theta}=\theta t+L_{t}$ is estimated with rate $n^{\frac{1}{2}-\frac{1}{\alpha}}$ and Fisher information $\int_{\mathbb{R}} \frac{\varphi_{\alpha}^{\prime}(u)^{2}}{\varphi_{\alpha}(u)} \mathrm{d} u$ and that the parameter $\sigma$ of the process $X_{t}^{\sigma}=\sigma L_{t}$ is estimated with the usual rate $n^{-1 / 2}$ and Fisher information $\int_{\mathbb{R}} \frac{\left(\varphi_{\alpha}(u)+u \varphi_{\alpha}^{\prime}(u)\right)^{2}}{\varphi_{\alpha}(u)} \mathrm{d} u$.

ii) It is worth to notice that the information does not depend on the truncation function $\tau$, but depends on $\alpha$ through the Fisher information of the translated $\alpha$-stable process and multiplicative $\alpha$-stable process.

Theorem 2.4. With the notations and assumptions of Theorem 2.2, the following convergences hold:

$$
J_{n}(\beta) \stackrel{n \rightarrow \infty}{\longrightarrow} \mathcal{I}(\beta) \quad \text { in probability }
$$




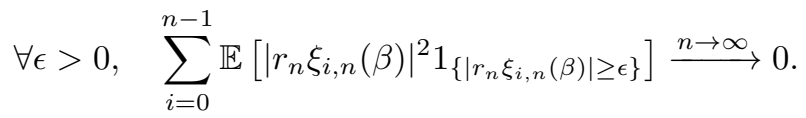

Theorem 2.5. We have the convergence in law

$$
J_{n}(\beta)^{\frac{1}{2}} N_{n}(\beta)=r_{n} \sum_{i=0}^{n-1} \xi_{i, n}(\beta) \Rightarrow \mathcal{N}(0, \mathcal{I}(\beta))
$$

where the limit variable is conditionally Gaussian and the convergence is stable with respect to the $\sigma$-field $\sigma\left(L_{s}, s \leq 1\right)$.

The stable convergence in law (2.6) and the convergence in probability (2.4) yield the convergence in law of the couple $\left(J_{n}(\beta), N_{n}(\beta)\right)$ :

$$
\left(J_{n}(\beta), N_{n}(\beta)\right) \Rightarrow(\mathcal{I}(\beta), N)
$$

where $N$ is a standard Gaussian variable independent of $\mathcal{I}(\beta)$. As a consequence of the asymptotic expansion given in Theorem 2.2 and the preceding limit theorems, we deduce the LAMN property.

Corollary 2.6. The family $\left(\mathbb{P}_{n}^{\beta}\right)$ satisfies the LAMN property with rate $r_{n}=\left(\begin{array}{cc}n^{\frac{1}{2}-\frac{1}{\alpha}} & 0 \\ 0 & n^{-\frac{1}{2}}\end{array}\right)$, and information $\mathcal{I}(\beta)$ given by $(2.3)$.

The rate of estimation of the drift parameter depends on $\alpha$ : when $\alpha$ tends to 2 , the rate is extremely low, however, when $\alpha$ goes to zero, it becomes high, especially for $\alpha<1$ where it is faster than the usual one $n^{-1 / 2}$. On the other hand, the rate of estimation of the volatility parameter $\sigma$ is $n^{-1 / 2}$ and does not depend on $\alpha$.

Before proceeding to the proof of these results, we discuss some extensions of our model that are not addressed in this paper.

- The Malliavin calculus used in this paper allows to consider the more general process

$$
X_{t}^{\beta}=x_{0}+\int_{0}^{t} b\left(X_{s}^{\beta}, \theta\right) \mathrm{d} s+\int_{0}^{t} c\left(X_{s-}^{\beta}, \sigma\right) \mathrm{d} L_{s}
$$

and based on the results given in [4] the Malliavin operators have explicit expressions. But the difficulty relies on the control of the Malliavin weights. Although explicit, these weights contain a lot of cumbersome terms especially the iterated weights involving the derivatives of the process with respect to the parameters $\theta$ and $\sigma$. These iterated weights (and their asymptotic behavior) are crucial to obtain the asymptotic behavior of the derivative of the transition density in small time (see [6]). The restriction to a constant coefficient $c$, assumed in this paper, permits to handle all these terms successfully. The theoretically possible extension to a general coefficient is still open.

- Unlike the papers $[1,2,12]$, our model does not contain an additional noise. Based on the structure of an additive model, the key point in these papers is that the density of the observed process can be written explicitly as a convolution between the Lévy process and the additional noise. Since we are dealing with a stochastic equation, this approach does not work anymore in our case and the introduction of an additional noise complicates significantly our model study.

- In contrast to the diffusion or jump-diffusion case, the interesting particularity of a pure jump process is that we can estimate the drift coefficient observing the process on a fixed time period $[0, T]$. It is important to stress that the estimation of $\theta$ is impossible without letting $T$ go to infinity if $\left(L_{t}\right)$ has a Brownian component. This is why we focus in this paper on the estimation of $(\theta, \sigma)$ from high frequency 
observations on the time interval $[0,1]$. The long time estimation problem $\left(n h_{n} \rightarrow \infty\right.$, where $n$ is the number of observations and $h_{n}$ the step between two consecutive observations) is also an interesting problem, but substantially different, that can certainly be treated with our methodology under ergodicity assumptions.

The remainder of the paper is devoted to the proofs of the main theorems above. The first step of our approach consists in studying the asymptotic Fisher information matrix by using Malliavin calculus techniques.

\section{The ASymptotic Fisher information matrix in SMALL Time}

\subsection{The asymptotic properties of the Fisher information matrix}

Our main concern in this section is to study the asymptotic properties of the Fisher information carried by the observation of the random variable $X_{1 / n}^{\beta}$. We recall the definition of the Fisher information matrix:

$$
\mathcal{I}^{n, \beta, x_{0}}=\left(\begin{array}{ll}
\mathcal{I}_{11}^{n, \beta, x_{0}} & \mathcal{I}_{12}^{n, \beta, x_{0}} \\
\mathcal{I}_{12}^{n, \beta, x_{0}} & \mathcal{I}_{22}^{n, \beta, x_{0}}
\end{array}\right)
$$

where

$$
\begin{aligned}
\mathcal{I}_{11}^{n, \beta, x_{0}}=\mathbb{E}\left[\left(\frac{\partial_{\theta} p_{\frac{1}{n}}^{\beta}\left(x_{0}, X_{1 / n}^{\beta}\right)}{p_{\frac{1}{n}}^{\beta}\left(x_{0}, X_{1 / n}^{\beta}\right)}\right)^{2}\right], \quad \mathcal{I}_{12}^{n, \beta, x_{0}}=\mathbb{E}\left[\left(\frac{\partial_{\theta} p_{\frac{1}{n}}^{\beta}\left(x_{0}, X_{1 / n}^{\beta}\right)}{p_{\frac{1}{n}}^{\beta}\left(x_{0}, X_{1 / n}^{\beta}\right)} \frac{\partial_{\sigma} p_{\frac{1}{n}}^{\beta}\left(x_{0}, X_{1 / n}^{\beta}\right)}{p_{\frac{1}{n}}^{\beta}\left(x_{0}, X_{1 / n}^{\beta}\right)}\right)\right], \\
\mathcal{I}_{22}^{n, \beta, x_{0}}=\mathbb{E}\left[\left(\frac{\partial_{\sigma} p_{\frac{1}{n}}^{\beta}\left(x_{0}, X_{1 / n}^{\beta}\right)}{p_{\frac{1}{n}}^{\beta}\left(x_{0}, X_{1 / n}^{\beta}\right)}\right)^{2}\right] .
\end{aligned}
$$

The following proposition gives the asymptotic behavior of the Fisher information based on the observation of the random variable $X_{1 / n}^{\beta}$ as $\frac{1}{n}$ tends to zero.

Theorem 3.1. Let $\left(\beta_{n}\right)$ be a sequence such that $\beta_{n} \stackrel{n \rightarrow \infty}{\longrightarrow} \beta, Q \subset \mathbb{R} \times(0, \infty)$ a compact set and $r_{n}=$ $\left(\begin{array}{cc}n^{\frac{1}{2}-\frac{1}{\alpha}} & 0 \\ 0 & n^{-\frac{1}{2}}\end{array}\right)$ then

i) $n r_{n} \mathcal{I}^{n, \beta_{n}, x_{0}} r_{n} \stackrel{n \rightarrow \infty}{\longrightarrow}\left(\begin{array}{cc}\frac{1}{\sigma^{2}} \partial_{\theta} b\left(x_{0}, \theta\right)^{2} \int_{\mathbb{R}} \frac{\varphi_{\alpha}^{\prime}(u)^{2}}{\varphi_{\alpha}(u)} \mathrm{d} u & 0 \\ 0 & \frac{1}{\sigma^{2}} \int_{\mathbb{R}} \frac{\left[\varphi_{\alpha}(u)+u \varphi_{\alpha}^{\prime}(u)\right]^{2}}{\varphi_{\alpha}(u)} \mathrm{d} u\end{array}\right)$

and this convergence is uniform with respect to $x_{0}$.

ii) $\forall p \geq 1, \quad \sup _{n, \beta \in Q, x_{0}} \mathbb{E}\left|n^{1 / 2} r_{n} \frac{\nabla_{\beta} p_{\frac{1}{n}}^{\beta}\left(x_{0}, X_{\frac{1}{n}}^{\beta}\right)}{p_{\frac{1}{n}}^{\beta}\left(x_{0}, X_{\frac{1}{n}}^{\beta}\right)}\right|^{p}<\infty$.

As a consequence of ii) with $p=2$, we deduce immediately $\sup _{n, \beta \in Q, x_{0}} n^{2-2 / \alpha} \mathcal{I}_{11}^{n, \beta, x_{0}}<\infty$, $\sup _{n, \beta \in Q, x_{0}} \mathcal{I}_{22}^{n, \beta, x_{0}}<\infty$ and from Cauchy-Schwarz inequality $\sup _{n, \beta \in Q, x_{0}} n^{1-1 / \alpha} \mathcal{I}_{12}^{n, \beta, x_{0}}<\infty$.

Remark 3.2. From Theorem 3.1, we see that the Fisher information contained in one observation is of magnitude $n^{-1} r_{n}^{-2}$ and the Fisher information based on $n$ observations is of magnitude $r_{n}^{-2}$. This is consistent with the fact that $r_{n}$ is the rate in the LAMN property stated in Corollary 2.6.

The rest of this section is devoted to the proof of Theorem 3.1. 


\subsection{Proof of Theorem 3.1}

The proof of Theorem 3.1 relies on a representation of the score function using Malliavin calculus initiated by Gobet (see $[8,9])$ and adapted to our context in $[6]$.

This representation is established after a rescaling that we describe in the next subsection.

\subsubsection{Rescaling and representation of the score function using Malliavin calculus}

We consider $\mu^{e}(\mathrm{~d} t, \mathrm{~d} z, \mathrm{~d} u)$ a Poisson measure on $[0, \infty) \times \mathbb{R} \times[0,1]$ with compensating measure $v^{e}(\mathrm{~d} t, \mathrm{~d} z, \mathrm{~d} u)=\mathrm{d} t 1_{|z| \neq 0} \frac{\mathrm{d} z}{|z|^{1+\alpha}} \mathrm{d} u$ and for $n \geq 1$, we define the Poisson random measure $\mu^{(n)}$ by

$$
\forall A \subset[0, \infty) \times \mathbb{R}, \quad \mu^{(n)}(A)=\int_{[0, \infty)} \int_{\mathbb{R}} \int_{[0,1]} 1_{A}(t, z) 1_{\left\{u \leq \tau\left(\frac{z}{n^{1 / \alpha}}\right)\right\}} \mu^{e}(\mathrm{~d} t, \mathrm{~d} z, \mathrm{~d} u) .
$$

We note that the compensator of $\mu^{(n)}(\mathrm{d} t, \mathrm{~d} z)$ is $v^{(n)}(\mathrm{d} t, \mathrm{~d} z)=\mathrm{d} t \times \tau\left(\frac{z}{n^{1 / \alpha}}\right) 1_{|z| \neq 0} \frac{\mathrm{d} z}{|z|^{1+\alpha}}:=\mathrm{d} t \times F_{n}(z) \mathrm{d} z$ and the compensated Poisson random measure by $\tilde{\mu}^{(n)}(\mathrm{d} t, \mathrm{~d} z)=\mu^{(n)}(\mathrm{d} t, \mathrm{~d} z)-v^{(n)}(\mathrm{d} t, \mathrm{~d} z)$.

We define the process $\left(L_{t}^{n}\right)$ by:

$$
L_{t}^{n}=\int_{0}^{t} \int_{\left[-n^{1 / \alpha}, n^{1 / \alpha}\right]} z \tilde{\mu}^{(n)}(\mathrm{d} s, \mathrm{~d} z)+\int_{0}^{t} \int_{\left[-n^{1 / \alpha}, n^{1 / \alpha}\right]^{c}} z \mu^{(n)}(\mathrm{d} s, \mathrm{~d} z) .
$$

We observe that the process $\left(L_{t / n}\right)$ (recall $\left.\mathbf{H}_{1}\left(b_{i}\right)\right)$ equals in law $\left(\frac{1}{n^{1 / \alpha}} L_{t}^{n}\right)$ since the associated Poisson measures have the same compensator. Moreover, when $n$ grows, we can show that the process $\left(L_{t}^{n}\right)$ converges almost surely to an $\alpha$-stable process defined by

$$
L_{t}^{\alpha}=\int_{0}^{t} \int_{[-1,1]} z \tilde{\mu}(\mathrm{d} s, \mathrm{~d} z)+\int_{0}^{t} \int_{[-1,1]^{c}} z \mu(\mathrm{d} s, \mathrm{~d} z),
$$

where $\mu$ is the Poisson random measure defined by,

$$
\forall A \subset[0, \infty) \times \mathbb{R}, \quad \mu(A)=\int_{[0, \infty)} \int_{\mathbb{R}} \int_{[0,1]} 1_{A}(t, z) \mu^{e}(\mathrm{~d} t, \mathrm{~d} z, \mathrm{~d} u) .
$$

The compensator of $\mu(\mathrm{d} t, \mathrm{~d} z)$ is $v(\mathrm{~d} t, \mathrm{~d} z)=\mathrm{d} t \times 1_{|z| \neq 0} \frac{\mathrm{d} z}{|z|^{1+\alpha}}$ and we denote the compensated Poisson random measure by $\tilde{\mu}(\mathrm{d} t, \mathrm{~d} z)=\mu(\mathrm{d} t, \mathrm{~d} z)-v(\mathrm{~d} t, \mathrm{~d} z)$.

It is important to note that $L^{n}$ and the $\alpha$-stable process $L^{\alpha}$ are defined on the same probability space (this property is crucial in our method to study the convergence of the Fisher information $\left.\mathcal{I}^{n, \beta, x_{0}}\right)$. The connection between $L^{n}$ and $L^{\alpha}$ is given more clearly by the following lemma.

Lemma 3.3 (Lem. 3.1 in [6]). On the event $A_{n}=\left\{\mu\left(\left\{(t, z)|0 \leq t \leq 1| z \mid, \geq n^{1 / \alpha}\right\}\right)=0\right\}$, we have

$$
\mu^{(n)}=\mu, \quad L_{t}^{n}=L_{t}^{\alpha},
$$

and $\mathbb{P}\left(A_{n}\right)=1+O(1 / n)$.

Furthermore, let $\left(f_{n}\right)_{n \in \mathbb{N}}$ and $f$ be measurable functions from $\Omega \times[0,1] \times \mathbb{R}$ to $\mathbb{R}$ such that there exists $C$ with $\mathbb{P}(C)=1$ and $\forall \omega \in C, \forall s \in[0,1], \forall|z|>1 f_{n}(\omega, s, z) \stackrel{n \rightarrow \infty}{\longrightarrow} f(\omega, s, z)$. Then

$$
\int_{0}^{1} \int_{|z|>1} f_{n}(\omega, s, z) \mu^{(n)}(\mathrm{d} s, \mathrm{~d} z) \underset{a . s .}{\stackrel{n \rightarrow \infty}{\longrightarrow}} \int_{0}^{1} \int_{|z|>1} f(\omega, s, z) \mu(\mathrm{d} s, \mathrm{~d} z) .
$$


Moreover, we have $\sup _{t \in[0,1]}\left|L_{t}^{n}-L_{t}^{\alpha}\right| \underset{a . s}{\stackrel{n \rightarrow \infty}{\longrightarrow}} 0$.

We now consider the process $\left(\bar{Y}_{t}^{n, \beta, x_{0}}\right)$ solution to the equation

$$
\bar{Y}_{t}^{n, \beta, x_{0}}=x_{0}+\frac{1}{n} \int_{0}^{t} b\left(\bar{Y}_{s}^{n, \beta, x_{0}}, \theta\right) \mathrm{d} s+\frac{\sigma}{n^{1 / \alpha}} L_{t}^{n} \quad t \in[0,1] .
$$

From the construction of $L^{n},\left(X_{\frac{t}{n}}^{\beta}\right)_{t \in[0,1]}$ equals in law $\left(\bar{Y}_{t}^{n, \beta, x_{0}}\right)_{t \in[0,1]}$. Let $q^{n, \beta, x_{0}}$ be the density of $\bar{Y}_{1}^{n, \beta, x_{0}}$ then the connection between the densities of $X_{\frac{1}{n}}^{\beta}$ and $\bar{Y}_{1}^{n, \beta, x_{0}}$ is given by

$$
p_{1 / n}^{\beta}\left(x_{0}, x\right)=q^{n, \beta, x_{0}}(x) .
$$

We remark also that $\left(\bar{Y}_{t}^{n, \beta, x_{0}}\right)_{t}$ admits derivatives with respect to the parameters $\theta$ and $\sigma$, denoted by $\left(\partial_{\theta} Y_{t}^{\beta}\right)_{t}$ and $\left(\partial_{\sigma} Y_{t}^{\beta}\right)_{t}$, respectively. With these notations, we have the following representation.

Proposition 3.4 (Thm. 6.2 in [6]). Let $q^{n, \beta, x_{0}}$ be the density of $\bar{Y}_{1}^{n, \beta, x_{0}}$ then we have the representation of the logarithmic derivative of the density as a conditional expectation:

$$
\frac{\nabla_{\beta} p_{\frac{1}{n}}^{\beta}}{p_{\frac{1}{n}}^{\beta}}\left(x_{0}, u\right)=\frac{\nabla_{\beta} q^{n, \beta, x_{0}}}{q^{n, \beta, x_{0}}}(u)=\left(\begin{array}{c}
\frac{\partial_{\theta} q^{n, \beta, x_{0}}}{q^{n, \beta, x_{0}}}(u) \\
\frac{\partial_{\sigma} q^{n, \beta, x_{0}}}{q^{n, \beta, x_{0}}}(u)
\end{array}\right)=\mathbb{E}\left(\mathcal{H}_{\bar{Y}_{1}^{n, \beta, x_{0}}}\left(\nabla_{\beta} \bar{Y}_{1}^{n, \beta, x_{0}}\right) \mid \bar{Y}_{1}^{n, \beta, x_{0}}=u\right)
$$

with

$$
\mathcal{H}_{\bar{Y}_{1}^{n, \beta, x_{0}}}\left(\nabla_{\beta} \bar{Y}_{1}^{n, \beta, x_{0}}\right)=\left(\begin{array}{l}
\mathcal{H}_{\bar{Y}_{1}^{n, \beta, x_{0}}}\left(\partial_{\theta} \bar{Y}_{1}^{n, \beta, x_{0}}\right) \\
\mathcal{H}_{\bar{Y}_{1}^{n, \beta, x_{0}}}\left(\partial_{\sigma} \bar{Y}_{1}^{n, \beta, x_{0}}\right)
\end{array}\right)
$$

The Malliavin weight $\mathcal{H}_{\bar{Y}_{1}^{n, \beta, x_{0}}}\left(\nabla_{\beta} \bar{Y}_{1}^{n, \beta, x_{0}}\right)$ depends on the derivatives of $\bar{Y}_{1}^{n, \beta, x_{0}}$ with respect to $\theta$ and $\sigma$ and on Malliavin operators. Its explicit expression will be given in Section 6 (see (6.11)) after some recalling on Malliavin calculus.

\subsubsection{Intermediate lemmas}

In this section, we study the convergence of the Malliavin weight appearing in the representation of the score function. The limit of this Malliavin weight brings out an other weight denoted by $\mathcal{H}_{L^{\alpha}}(1)$ (given explicitly in (6.16)) that permits to represent $\varphi_{\alpha}^{\prime} / \varphi_{\alpha}$, where $\varphi_{\alpha}$ is the density of $L_{1}^{\alpha}$, as an expectation. This representation is not immediate since $L_{1}^{\alpha}$ does not belong to the domain of our Malliavin operators (see Sect. 6).

Lemma 3.5. We have the representation

$$
\frac{\varphi_{\alpha}^{\prime}(u)}{\varphi_{\alpha}(u)}=-\mathbb{E}\left[\mathcal{H}_{L^{\alpha}}(1) \mid L_{1}^{\alpha}=u\right]
$$

The connection between the weights $\mathcal{H}_{\bar{Y}_{1}^{n, \beta, x_{0}}}\left(\nabla_{\beta} \bar{Y}_{1}^{n, \beta, x_{0}}\right)$ and $\mathcal{H}_{L^{\alpha}}(1)$ is established in the next lemmas. The first lemma shows the convergence of the normalized iterated Malliavin weight $\mathcal{H}_{\bar{Y}_{1}^{n, \beta, x_{0}}}\left(\nabla_{\beta} \bar{Y}_{1}^{n, \beta, x_{0}}\right)$. 
Lemma 3.6. Let $\left(\beta_{n}\right)_{n \geq 1}$ be a sequence such that $\beta_{n} \stackrel{n \rightarrow \infty}{\longrightarrow} \beta$. Then, the following convergence holds uniformly with respect to $x_{0}$

$$
n^{1 / 2} r_{n} \mathcal{H}_{\bar{Y}_{1}^{n, \beta_{n}, x_{0}}}\left(\nabla_{\beta} \bar{Y}_{1}^{n, \beta_{n}, x_{0}}\right)=\left(\begin{array}{c}
n^{1-1 / \alpha} \mathcal{H}_{\bar{Y}_{1}^{n, \beta_{n}, x_{0}}}\left(\partial_{\theta} \bar{Y}_{1}^{n, \beta_{n}, x_{0}}\right) \\
\mathcal{H}_{\bar{Y}_{1}^{n, \beta_{n}, x_{0}}}\left(\partial_{\sigma} \bar{Y}_{1}^{n, \beta_{n}, x_{0}}\right)
\end{array}\right) \underset{L^{p}, \forall p \geq 1}{\longrightarrow}\left(\begin{array}{c}
\frac{1}{\sigma} \partial_{\theta} b\left(x_{0}, \theta\right) \mathcal{H}_{L^{\alpha}}(1) \\
\frac{1}{\sigma}\left(L_{1}^{\alpha} \mathcal{H}_{L^{\alpha}}(1)-1\right)
\end{array}\right)
$$

where $L_{1}^{\alpha}$ is defined by (3.3). Moreover, for any compact subset $Q \subset \mathbb{R} \times(0, \infty)$,

$$
\forall p \geq 1, \quad \sup _{n, \beta \in Q, x_{0}} \mathbb{E}\left|n^{1 / 2} r_{n} \mathcal{H}_{\bar{Y}_{1}^{n, \beta, x_{0}}}\left(\nabla_{\beta} \bar{Y}_{1}^{n, \beta, x_{0}}\right)\right|^{p}<\infty
$$

The next two lemmas are related to a continuity property with respect to the conditioning variable, in a conditional expectation.

Lemma 3.7. Let $\left(\beta_{n}\right)_{n \geq 1}$ be a sequence such that $\beta_{n} \stackrel{n \rightarrow \infty}{\longrightarrow} \beta$. Then, the following convergence holds uniformly with respect to $x_{0}$.
i) $n^{2-2 / \alpha} \mathbb{E}\left[\mathbb{E}\left[\mathcal{H}_{\bar{Y}_{1}^{n, \beta_{n}, x_{0}}}\left(\partial_{\theta} \bar{Y}_{1}^{n, \beta_{n}, x_{0}}\right) \mid \bar{Y}_{1}^{n, \beta_{n}, x_{0}}\right]^{2}\right] \stackrel{n \rightarrow \infty}{\longrightarrow} \frac{1}{\sigma^{2}}\left[\partial_{\theta} b\left(x_{0}, \theta\right)\right]^{2} \mathbb{E}\left[\mathbb{E}\left[\mathcal{H}_{L^{\alpha}}(1) \mid L_{1}^{\alpha}\right]^{2}\right]$,
ii) $\mathbb{E}\left[\mathbb{E}\left[\mathcal{H}_{\bar{Y}_{1}^{n, \beta_{n}, x_{0}}}\left(\partial_{\sigma} \bar{Y}_{1}^{n, \beta_{n}, x_{0}}\right) \mid \bar{Y}_{1}^{n, \beta_{n}, x_{0}}\right]^{2}\right] \stackrel{n \rightarrow \infty}{\longrightarrow} \frac{1}{\sigma^{2}} \mathbb{E}\left[\mathbb{E}\left[L_{1}^{\alpha} \mathcal{H}_{L^{\alpha}}(1)-1 \mid L_{1}^{\alpha}\right]^{2}\right]$,

Lemma 3.8. Let $\left(\beta_{n}\right)_{n \geq 1}$ be a sequence such that $\beta_{n} \stackrel{n \rightarrow \infty}{\longrightarrow} \beta$. Then, the following convergence holds uniformly with respect to $x_{0}$,

$$
\begin{aligned}
n^{1-1 / \alpha} \mathbb{E}\left[\mathbb{E}\left[\mathcal{H}_{\bar{Y}_{1}^{n, \beta_{n}, x_{0}}}\left(\partial_{\theta} \bar{Y}_{1}^{n, \beta_{n}, x_{0}}\right) \mid \bar{Y}_{1}^{n, \beta_{n}, x_{0}}\right] \mathbb{E}\left[\mathcal{H}_{\bar{Y}_{1}^{n, \beta_{n}, x_{0}}}\left(\partial_{\sigma} \bar{Y}_{1}^{n, \beta_{n}, x_{0}}\right) \mid \bar{Y}_{1}^{n, \beta_{n}, x_{0}}\right]\right] \\
\stackrel{n \rightarrow \infty}{\longrightarrow} \frac{1}{\sigma^{2}} \mathbb{E}\left[\mathbb{E}\left[\mathcal{H}_{L^{\alpha}}(1) \mid L_{1}^{\alpha}\right] \mathbb{E}\left[L_{1}^{\alpha} \mathcal{H}_{L^{\alpha}}(1)-1 \mid L_{1}^{\alpha}\right]\right]
\end{aligned}
$$

The proofs of the above lemmas are very technical. They are postponed to Section 6. Admitting these intermediate results, we can proceed to the proof of Theorem 3.1.

\subsubsection{Proof of Theorem 3.1}

Proof. i) We need to prove that for $\left(\beta_{n}\right)$ a sequence such that $\beta_{n} \stackrel{n \rightarrow \infty}{\longrightarrow} \beta$ and $r_{n}=\left(\begin{array}{cc}n^{\frac{1}{2}-\frac{1}{\alpha}} & 0 \\ 0 & n^{-\frac{1}{2}}\end{array}\right)$ we have

$$
n r_{n} \mathcal{I}^{n, \beta_{n}, x_{0}} r_{n} \stackrel{n \rightarrow \infty}{\longrightarrow}\left(\begin{array}{cc}
\frac{1}{\sigma^{2}} \partial_{\theta} b\left(x_{0}, \theta\right)^{2} \int_{\mathbb{R}} \frac{\varphi_{\alpha}^{\prime}(u)^{2}}{\varphi_{\alpha}(u)} \mathrm{d} u & 0 \\
0 & \frac{1}{\sigma^{2}} \int_{\mathbb{R}} \frac{\left[\varphi_{\alpha}(u)+u \varphi_{\alpha}^{\prime}(u)\right]^{2}}{\varphi_{\alpha}(u)} \mathrm{d} u
\end{array}\right)
$$

and that this convergence is uniform with respect to $x_{0}$.

Since $n r_{n} \mathcal{I}^{n, \beta_{n}, x_{0}} r_{n}=\left(\begin{array}{cc}n^{2-\frac{2}{\alpha}} \mathcal{I}_{11}^{n, \beta_{n}, x_{0}} & n^{1-\frac{1}{\alpha}} \mathcal{I}_{12}^{n, \beta_{n}, x_{0}} \\ n^{1-\frac{1}{\alpha}} \mathcal{I}_{12}^{n, \beta_{n}, x_{0}} & \mathcal{I}_{22}^{n, \beta_{n}, x_{0}}\end{array}\right)$, the proof of the above convergence reduces to prove the convergence of each entries of the matrix. 
Convergence of $\boldsymbol{n}^{\mathbf{2 - 2 / \alpha}} \mathcal{I}_{\mathbf{1 1}}^{\boldsymbol{n}, \boldsymbol{\beta}_{\boldsymbol{n}}, \boldsymbol{x}_{0}}$. From (3.6) in Proposition 3.4, we have

$$
\begin{aligned}
n^{2-2 / \alpha} \mathcal{I}_{11}^{n, \beta_{n}, x_{0}}= & n^{2-2 / \alpha} \mathbb{E}\left[\mathbb{E}\left[\mathcal{H}_{\bar{Y}_{1}^{n, \beta_{n}, x_{0}}}\left(\partial_{\theta} \bar{Y}_{1}^{n, \beta_{n}, x_{0}}\right) \mid \bar{Y}_{1}^{n, \beta_{n}, x_{0}}\right]^{2}\right] \\
& \left.\stackrel{n \rightarrow \infty}{\longrightarrow} \frac{1}{\sigma^{2}} \partial_{\theta} b\left(x_{0}, \theta\right)^{2} \mathbb{E}\left[\mathbb{E}\left[\mathcal{H}_{L^{\alpha}}(1) \mid L_{1}^{\alpha}\right]^{2}\right], \quad \text { uniformly with respect to } x_{0} \text {, from Lemma } 3.7 i\right), \\
= & \frac{1}{\sigma^{2}} \partial_{\theta} b\left(x_{0}, \theta\right)^{2} \mathbb{E}\left[\frac{\varphi_{\alpha}^{\prime}\left(L_{1}^{\alpha}\right)^{2}}{\varphi_{\alpha}\left(L_{1}^{\alpha}\right)^{2}}\right] \quad \text { from (3.7) in Lemma } 3.5
\end{aligned}
$$

Convergence of $\mathcal{I}_{\mathbf{2} 2}^{\boldsymbol{n}, \boldsymbol{\beta}_{n}, \boldsymbol{x}_{\mathbf{0}}}$. We remark that from the representation (3.7), we can deduce the following representation

$$
\frac{\varphi_{\alpha}(u)+u \varphi_{\alpha}^{\prime}(u)}{\varphi_{\alpha}(u)}=-u \mathbb{E}\left[\left(\mathcal{H}_{L^{\alpha}}(1)\right) \mid L_{1}^{\alpha}=u\right]+1=-\mathbb{E}\left[\left(L_{1}^{\alpha} \mathcal{H}_{L^{\alpha}}(1)-1\right) \mid L_{1}^{\alpha}=u\right] .
$$

Furthermore, combining (3.6) and Lemma 3.7 ii), we have

$$
\begin{aligned}
\mathcal{I}_{22}^{n, \beta_{n}, x_{0}}= & \mathbb{E}\left[\mathbb{E}\left[\mathcal{H}_{\bar{Y}_{1}^{n, \beta_{n}, x_{0}}}\left(\partial_{\sigma} \bar{Y}_{1}^{n, \beta_{n}, x_{0}}\right) \mid \bar{Y}_{1}^{n, \beta_{n}, x_{0}}\right]^{2}\right], \\
& \stackrel{n \rightarrow \infty}{\longrightarrow} \frac{1}{\sigma^{2}} \mathbb{E}\left[\mathbb{E}\left[\left(L_{1}^{\alpha} \mathcal{H}_{L^{\alpha}}(1)-1\right) \mid L_{1}^{\alpha}\right]^{2}\right], \quad \text { uniformly with respect to } x_{0}, \\
= & \frac{1}{\sigma^{2}} \mathbb{E}\left[\frac{\left(\varphi_{\alpha}\left(L_{1}^{\alpha}\right)+L_{1}^{\alpha} \varphi_{\alpha}^{\prime}\left(L_{1}^{\alpha}\right)\right)^{2}}{\varphi_{\alpha}\left(L_{1}^{\alpha}\right)^{2}}\right] \quad \text { from }(3.10) .
\end{aligned}
$$

Convergence of $\boldsymbol{n}^{\mathbf{1 - 1 / \alpha}} \mathcal{I}_{12}^{n, \boldsymbol{\beta}_{n}, \boldsymbol{x}_{0}}$. We first recall the expression for the Fisher information

$$
\mathcal{I}_{12}^{n, \beta_{n}, x_{0}}=\mathbb{E}\left[\left(\frac{\partial_{\theta} p_{\frac{1}{n}}^{\beta_{n}}\left(x_{0}, X_{1 / n}^{\beta_{n}}\right)}{p_{\frac{1}{n}}^{\beta_{n}}\left(x_{0}, X_{1 / n}^{\beta_{n}}\right)} \frac{\partial_{\sigma} p_{\frac{1}{n}}^{\beta_{n}}\left(x_{0}, X_{1 / n}^{\beta_{n}}\right)}{p_{\frac{1}{n}}^{\beta_{n}}\left(x_{0}, X_{1 / n}^{\beta_{n}}\right)}\right)\right]
$$

then from (3.6) in Proposition 3.4 and Lemma 3.8 we have

$$
\begin{aligned}
n^{1-1 / \alpha} \mathcal{I}_{12}^{n, \beta_{n}, x_{0}}= & n^{1-1 / \alpha} \mathbb{E}\left[\mathbb{E}\left[\mathcal{H}_{\bar{Y}_{1}^{n, \beta_{n}, x_{0}}}\left(\partial_{\theta} \bar{Y}_{1}^{n, \beta_{n}, x_{0}}\right) \mid \bar{Y}_{1}^{n, \beta_{n}, x_{0}}\right] \mathbb{E}\left[\mathcal{H}_{\bar{Y}_{1}^{n, \beta_{n}, x_{0}}}\left(\partial_{\sigma} \bar{Y}_{1}^{n, \beta_{n}, x_{0}}\right) \mid \bar{Y}_{1}^{n, \beta_{n}, x_{0}}\right]\right] \\
& \stackrel{n \rightarrow \infty}{\longrightarrow} \frac{1}{\sigma^{2}} \mathbb{E}\left[\mathbb{E}\left[\mathcal{H}_{L^{\alpha}}(1) \mid L_{1}^{\alpha}\right] \mathbb{E}\left[L_{1}^{\alpha} \mathcal{H}_{L^{\alpha}}(1)-1 \mid L_{1}^{\alpha}\right]\right]
\end{aligned}
$$

On the other hand, from (3.7) and (3.10) we get

$$
\frac{\varphi_{\alpha}^{\prime}\left(L_{1}^{\alpha}\right)}{\varphi_{\alpha}\left(L_{1}^{\alpha}\right)}\left(\frac{\varphi_{\alpha}\left(L_{1}^{\alpha}\right)+L_{1}^{\alpha} \varphi_{\alpha}^{\prime}\left(L_{1}^{\alpha}\right)}{\varphi_{\alpha}\left(L_{1}^{\alpha}\right)}\right)=\mathbb{E}\left[\mathcal{H}_{L^{\alpha}}(1) \mid L_{1}^{\alpha}\right] \mathbb{E}\left[\left(L_{1}^{\alpha} \mathcal{H}_{L^{\alpha}}(1)-1\right) \mid L_{1}^{\alpha}\right]
$$

Combining (3.11) with (3.12) gives

$$
n^{1-1 / \alpha} \mathcal{I}_{12}^{n, \beta_{n}, x_{0}} \stackrel{n \rightarrow \infty}{\longrightarrow} \frac{1}{\sigma^{2}} \int_{\mathbb{R}} \frac{\varphi_{\alpha}^{\prime}(u)\left[\varphi_{\alpha}(u)+u \varphi_{\alpha}^{\prime}(u)\right]}{\varphi_{\alpha}(u)} \mathrm{d} u=\frac{1}{\sigma^{2}} \int_{\mathbb{R}} \varphi_{\alpha}^{\prime}(u) \mathrm{d} u+\frac{1}{\sigma^{2}} \int_{\mathbb{R}} \frac{u\left(\varphi_{\alpha}^{\prime}(u)\right)^{2}}{\varphi_{\alpha}(u)} \mathrm{d} u=0,
$$

where we used the fact that $\varphi_{\alpha}$ is a symmetric function, and that the functions under the integral are odd. This completes the proof of part $i$ ). 
ii) Using (3.6) in Proposition 3.4 again and Jensen's inequality, we get:

$$
\mathbb{E}\left|n^{1 / 2} r_{n} \frac{\nabla_{\beta} p_{\frac{1}{n}}^{\beta}\left(x_{0}, X_{\frac{1}{n}}^{\beta}\right)}{p_{\frac{1}{n}}^{\beta}\left(x_{0}, X_{\frac{1}{n}}^{\beta}\right)}\right|^{p} \leq \mathbb{E}\left|n^{1 / 2} r_{n} \mathcal{H}_{\bar{Y}_{1}^{n, \beta, x_{0}}}\left(\nabla_{\beta} \bar{Y}_{1}^{n, \beta, x_{0}}\right)\right|^{p},
$$

and the result follows from Lemma 3.6.

This achieves the proof of Theorem 3.1.

\section{Proof of the Asymptotic expansion of the LiKelihood}

(Thms. 2.2-2.4)

The aim of this section is to prove the asymptotic expansion of the log-likelihood function, stated in Theorem 2.2 as well as the convergence given in Theorem 2.4. The proof of Theorem 2.2 is based essentially on the $L^{2}$-regularity property of the transition density $p_{1 / n}^{\beta}(x, y)$. From Jeganathan's article [14], the following four conditions A1-A4 are sufficient to get the expansion (2.2) of Theorem 2.2.

We recall the notation $\xi_{i, n}(\beta)=\left(\begin{array}{c}\frac{\partial_{\theta} p_{\frac{1}{n}}^{\beta}}{p_{\frac{1}{n}}^{\beta}}\left(X_{\frac{i}{n}}^{\beta}, X_{\frac{i+1}{n}}^{\beta}\right) \\ \frac{\partial_{\sigma} p_{\frac{1}{n}}^{\beta}}{p_{\frac{1}{n}}^{\beta}}\left(X_{\frac{i}{n}}^{\beta}, X_{\frac{i+1}{n}}^{\beta}\right)\end{array}\right)$ and we denote $\chi_{n}(\beta, x, y)=\left(\begin{array}{c}\frac{\partial_{\theta} p_{\frac{1}{n}}^{\beta}(x, y)}{p_{\frac{1}{n}}^{\beta}(x, y)^{1 / 2}} \\ \frac{\partial_{\sigma} p_{\frac{1}{n}}^{\beta}(x, y)}{p_{\frac{1}{n}}^{\beta}(x, y)^{1 / 2}}\end{array}\right)$.

\section{A1. $L^{2}$-regularity}

$$
\sum_{j=1}^{n} \mathbb{E}\left[\int_{\mathbb{R}}\left\{p_{\frac{1}{n}}^{\beta+r_{n} h}\left(X_{\frac{j-1}{n}}^{\beta}, y\right)^{1 / 2}-p_{\frac{1}{n}}^{\beta}\left(X_{\frac{j-1}{n}}^{\beta}, y\right)^{1 / 2}-\frac{1}{2} h^{T} r_{n} \chi_{n}\left(\beta, X_{\frac{j-1}{n}}^{\beta}, y\right)\right\}^{2} \mathrm{~d} y\right] \stackrel{n \rightarrow \infty}{\longrightarrow} 0 .
$$

A2.

$$
J_{n}(\beta)=r_{n} \sum_{i=0}^{n-1} \mathbb{E}\left[\xi_{i, n}(\beta) \xi_{i, n}^{T}(\beta) \mid \mathcal{F}_{i / n}\right] r_{n} \stackrel{n \rightarrow \infty}{\longrightarrow} \mathcal{I}(\beta) \quad(>0 \quad \text { a.e. }), \quad \text { in probability. }
$$

A3.

$$
\forall \epsilon>0, \quad \sum_{i=0}^{n-1} \mathbb{E}\left[\left|r_{n} \xi_{i, n}(\beta)\right|^{2} 1_{\left\{\left|r_{n} \xi_{i, n}(\beta)\right| \geq \epsilon\right\}} \stackrel{n \rightarrow \infty}{\longrightarrow} 0\right.
$$

A4.

$$
\sup _{n} \sum_{i=0}^{n} \mathbb{E}\left(\left|r_{n} \xi_{i, n}(\beta) \xi_{i, n}(\beta)^{T} r_{n}\right|\right) \leq C, \quad \text { for a strictly positive constant } C .
$$

The condition A1 is proved in Section 4.1 and A2 and A3 are proved in Section 4.2. The condition A4 is immediate from Theorem $3.1 \mathrm{ii}$ ) since

$$
n \mathbb{E}\left(r_{n} \xi_{i, n}(\beta) \xi_{i, n}(\beta)^{T} r_{n}\right)=\mathbb{E}\left(\begin{array}{cc}
n^{2-\frac{2}{\alpha}} \mathcal{I}_{11}^{n, \beta, X_{i / n}^{\beta}} & n^{1-\frac{1}{\alpha}} \mathcal{I}_{12}^{n, \beta, X_{i / n}^{\beta}} \\
n^{1-\frac{1}{\alpha}} \mathcal{I}_{12}^{n, \beta, X_{i / n}^{\beta}} & \mathcal{I}_{22}^{n, \beta, X_{i / n}^{\beta}}
\end{array}\right)
$$


Note that these conditions do not imply the stable convergence in law (2.6) since in our framework the filtration $\left(\mathcal{F}_{\frac{i}{n}}\right)_{i}$ does not satisfy the "nested condition" (see Thm. 3.2 in [11]). The proof of the stable convergence in law will be given in Section 5 .

\subsection{Proof of the condition A1 (the $L^{2}$-regularity condition)}

Following [5], the crucial point of the proof is the asymptotic behavior of the transition density of $X_{t}^{\beta}$ established in [6] and recalled below.

Theorem 4.1 (Thm. 2.1 in [6]). We assume that $\boldsymbol{H}_{1}$ holds. Let $\left(\varsigma_{t}^{n, \theta, x_{0}}\right)$ be the solution to the ordinary differential equation

$$
\varsigma_{t}^{n, \theta, x_{0}}=x_{0}+\frac{1}{n} \int_{0}^{t} b\left(\varsigma_{s}^{n, \theta, x_{0}}, \theta\right) \mathrm{d} s \quad t \in[0,1]
$$

Let $\left(\beta_{n}\right)_{n \geq 1}$ be a sequence such that $\beta_{n} \stackrel{n \rightarrow \infty}{\longrightarrow} \beta$ then for all $\left(x_{0}, u\right) \in \mathbb{R}^{2}$,

i) $\frac{\sigma_{n}}{n^{1 / \alpha}} p_{\frac{1}{n}}^{\beta_{n}}\left(x_{0}, \frac{u \sigma_{n}}{n^{1 / \alpha}}+\varsigma_{1}^{n, \theta_{n}, x_{0}}\right) \stackrel{n \rightarrow \infty}{\longrightarrow} \varphi_{\alpha}(u)$,
ii) $\sup _{u \in \mathbb{R}} \sup _{n} \frac{\sigma_{n}}{n^{1 / \alpha}} p_{\frac{1}{n}}^{\beta_{n}}\left(x_{0}, \frac{u \sigma_{n}}{n^{1 / \alpha}}+\varsigma_{1}^{n, \theta_{n}, x_{0}}\right)<\infty$,

where $\varphi_{\alpha}$ is the density of $L_{1}^{\alpha}$, a centered $\alpha$-stable Lévy process.

Theorem 4.2 (Thm. 2.2 in [6]). Under the assumptions of Theorem 4.1,

$$
\begin{aligned}
& \text { i) } \frac{\sigma_{n}^{2}}{n^{\frac{2}{\alpha}-1}} \partial_{\theta} p_{\frac{1}{n}}^{\beta_{n}}\left(x_{0}, \frac{u \sigma_{n}}{n^{1 / \alpha}}+\varsigma_{1}^{n, \theta_{n}, x_{0}}\right) \stackrel{n \rightarrow \infty}{\longrightarrow}-\partial_{\theta} b\left(x_{0}, \theta\right) \times \varphi_{\alpha}^{\prime}(u), \\
& \frac{\sigma_{n}^{2}}{n^{1 / \alpha}} \partial_{\sigma} p_{\frac{1}{n}}^{\beta_{n}}\left(x_{0}, \frac{u \sigma_{n}}{n^{1 / \alpha}}+\varsigma_{1}^{n, \theta_{n}, x_{0}}\right) \stackrel{n \rightarrow \infty}{\longrightarrow}-\varphi_{\alpha}(u)-u \varphi_{\alpha}^{\prime}(u), \\
& \text { ii) } \sup _{u \in \mathbb{R}} \sup _{n}\left|\frac{\sigma_{n}^{2}}{n^{\frac{2}{\alpha}-1}} \partial_{\theta} p_{\frac{1}{n}}^{\beta_{n}}\left(x_{0}, \frac{u \sigma_{n}}{n^{1 / \alpha}}+\varsigma_{1}^{n, \theta_{n}, x_{0}}\right)\right|<\infty, \\
& \sup _{u \in \mathbb{R}} \sup _{n}\left|\frac{\sigma_{n}^{2}}{n^{1 / \alpha}} \partial_{\sigma} p_{\frac{1}{n}}^{\beta_{n}}\left(x_{0}, \frac{u \sigma_{n}}{n^{1 / \alpha}}+\varsigma_{1}^{n, \theta_{n}, x_{0}}\right)\right|<\infty .
\end{aligned}
$$

Proof of A1. By the change of variable $y=\frac{u \sigma}{n^{1 / \alpha}}+\varsigma_{1}^{n, \theta, X^{\beta}}{ }_{\frac{j-1}{n}}$ proving A1 is equivalent to show:

$$
\frac{1}{n} \sum_{j=1}^{n} \int_{\mathbb{R}} \mathbb{E}\left[\left\{f_{n}\left(X_{\frac{j-1}{n}}^{\beta}, u\right)-g_{n}\left(X_{\frac{j-1}{n}}^{\beta}, u\right)\right\}^{2}\right] \mathrm{d} u \stackrel{n \rightarrow \infty}{\longrightarrow} 0
$$

where

$$
\begin{aligned}
& f_{n}(x, u)=\sqrt{\sigma} n^{1 / 2-1 / 2 \alpha}\left[p_{\frac{1}{n}}^{\beta+r_{n} h}\left(x, \frac{u \sigma}{n^{1 / \alpha}}+\varsigma_{1}^{n, \theta, x}\right)^{1 / 2}-p_{\frac{1}{n}}^{\beta}\left(x, \frac{u \sigma}{n^{1 / \alpha}}+\varsigma_{1}^{n, \theta, x}\right)^{1 / 2}\right], \\
& g_{n}(x, u)=\frac{\sqrt{\sigma}}{2} n^{1 / 2-1 / 2 \alpha}\left(r_{n} h\right)^{T} \chi_{n}\left(\beta, x, \frac{u \sigma}{n^{1 / \alpha}}+\varsigma_{1}^{n, \theta, x}\right) .
\end{aligned}
$$

Following the proof of Proposition 8 in [5], the next three properties are sufficient to prove (4.2).

1. There exists a function $f$ such that,

$$
\begin{aligned}
& \forall x, u, \quad f_{n}(x, u) \stackrel{n \rightarrow \infty}{\longrightarrow} f(x, u), \\
& g_{n}(x, u) \stackrel{n \rightarrow \infty}{\longrightarrow} f(x, u) .
\end{aligned}
$$


2. We have for all $x$,

$$
\begin{aligned}
\limsup _{n} \int_{\mathbb{R}} f_{n}(x, u)^{2} \mathrm{~d} u & \leq \int_{\mathbb{R}} f(x, u)^{2} \mathrm{~d} u, \\
\limsup _{n} \int_{\mathbb{R}} g_{n}(x, u)^{2} \mathrm{~d} u & \leq \int_{\mathbb{R}} f(x, u)^{2} \mathrm{~d} u .
\end{aligned}
$$

3. We have

$$
\begin{aligned}
& \sup _{x, n} \int_{\mathbb{R}} f_{n}(x, u)^{2} \mathrm{~d} u<\infty, \\
& \sup _{x, n} \int_{\mathbb{R}} g_{n}(x, u)^{2} \mathrm{~d} u<\infty .
\end{aligned}
$$

We now need to check the validity of the conditions (4.3)-(4.5).

We start with the proof of the condition (4.3).

We recall that $r_{n}=\left(\begin{array}{cc}n^{\frac{1}{2}-\frac{1}{\alpha}} & 0 \\ 0 & n^{-\frac{1}{2}}\end{array}\right)$ and $h=\left(h_{1}, h_{2}\right)^{T} \in \mathbb{R}^{2}$ then by a simple computation we have,

$$
g_{n}(x, u)=\frac{\sqrt{\sigma}}{2} n^{1-\frac{3}{2 \alpha}} h_{1} \frac{\partial_{\theta} p_{\frac{1}{n}}^{\beta}\left(x, \frac{u \sigma}{n^{1 / \alpha}}+\varsigma_{1}^{n, \theta, x}\right)}{p_{\frac{1}{n}}^{\beta}\left(x, \frac{u \sigma}{n^{1 / \alpha}}+\varsigma_{1}^{n, \theta, x}\right)^{1 / 2}}+\frac{\sqrt{\sigma}}{2} n^{\frac{-1}{2 \alpha}} h_{2} \frac{\partial_{\sigma} p_{\frac{1}{n}}^{\beta}\left(x, \frac{u \sigma}{n^{1 / \alpha}}+\varsigma_{1}^{n, \theta, x}\right)}{p_{\frac{1}{n}}^{\beta}\left(x, \frac{u \sigma}{n^{1 / \alpha}}+\varsigma_{1}^{n, \theta, x}\right)^{1 / 2}} .
$$

From Theorems 4.1 and 4.2 , we see that

$$
g_{n}(x, u) \stackrel{n \rightarrow \infty}{\longrightarrow} f(x, u):=-\frac{h_{1}}{2 \sigma} \partial_{\theta} b(x, \theta) \frac{\varphi_{\alpha}^{\prime}(u)}{\varphi_{\alpha}(u)^{1 / 2}}-\frac{h_{2}}{2 \sigma} \frac{\left(\varphi_{\alpha}(u)+u \varphi_{\alpha}^{\prime}(u)\right)}{\varphi_{\alpha}(u)^{1 / 2}} .
$$

Let $m_{t}=p_{\frac{1}{n}}^{\beta+r_{n} h t}\left(x, \frac{u \sigma}{n^{1 / \alpha}}+\varsigma_{1}^{n, \theta, x}\right), t \in[0,1]$, then we can rewrite $f_{n}(x, u)$ as

$$
f_{n}(x, u)=\sqrt{\sigma} n^{1 / 2-1 / 2 \alpha}\left[m_{1}^{1 / 2}-m_{0}^{1 / 2}\right]
$$

Using the mean value theorem, we get for some $s(x, u) \in(0,1)$

$$
f_{n}(x, u)=\frac{\sqrt{\sigma}}{2} n^{1 / 2-1 / 2 \alpha} \frac{m_{s(x, u)}^{\prime}}{\sqrt{m_{s(x, u)}}}=\frac{\sqrt{\sigma}}{2} n^{1 / 2-1 / 2 \alpha}\left(r_{n} h\right)^{T} \frac{\nabla_{\beta} p_{\frac{1}{n}}^{\beta_{n}}\left(x, \frac{u \sigma}{n^{1 / \alpha}}+\varsigma_{1}^{n, \theta, x}\right)}{p_{\frac{1}{n}}^{\beta_{n}}\left(x, \frac{u \sigma}{n^{1 / \alpha}}+\varsigma_{1}^{n, \theta, x}\right)^{1 / 2}},
$$

where $\beta_{n}=\beta+r_{n} s(x, u) h$.

From Theorems 4.1 and 4.2 , we also get that $f_{n}(x, u) \stackrel{n \rightarrow \infty}{\longrightarrow} f(x, u)$.

Now we prove the condition (4.4). 
We have

$$
\begin{aligned}
\int_{\mathbb{R}} g_{n}(x, u)^{2} \mathrm{~d} u= & \frac{\sigma h_{1}^{2}}{4} n^{2-3 / \alpha} \int_{\mathbb{R}} \frac{\partial_{\theta} p_{\frac{1}{n}}^{\beta}\left(x, \frac{u \sigma}{n^{1 / \alpha}}+\varsigma_{1}^{n, \theta, x}\right)^{2}}{p_{\frac{1}{n}}^{\beta}\left(x, \frac{u \sigma}{n^{1 / \alpha}}+\varsigma_{1}^{n, \theta, x}\right)} \mathrm{d} u+\frac{\sigma h_{2}^{2}}{4} n^{-1 / \alpha} \int_{\mathbb{R}} \frac{\partial_{\sigma} p_{\frac{1}{n}}^{\beta}\left(x, \frac{u \sigma}{n^{1 / \alpha}}+\varsigma_{1}^{n, \theta, x}\right)^{2}}{p_{\frac{1}{n}}^{\beta}\left(x, \frac{u \sigma}{n^{1 / \alpha}}+\varsigma_{1}^{n, \theta, x}\right)} \mathrm{d} u \\
& +\frac{\sigma h_{1} h_{2}}{2} n^{1-2 / \alpha} \int_{\mathbb{R}} \frac{\partial_{\theta} p_{\frac{1}{n}}^{\beta}\left(x, \frac{u \sigma}{n^{1 / \alpha}}+\varsigma_{1}^{n, \theta, x}\right)}{p_{\frac{1}{n}}^{\beta}\left(x, \frac{u \sigma}{n^{1 / \alpha}}+\varsigma_{1}^{n, \theta, x}\right)^{1 / 2}} \frac{\partial_{\sigma} p_{\frac{1}{n}}^{\beta}\left(x, \frac{u \sigma}{n^{1 / \alpha}}+\varsigma_{1}^{n, \theta, x}\right)}{p_{\frac{1}{n}}^{\beta}\left(x, \frac{u \sigma}{n^{1 / \alpha}}+\varsigma_{1}^{n, \theta, x}\right)^{1 / 2}} \mathrm{~d} u .
\end{aligned}
$$

From Theorem 3.1, we get

$$
\int_{\mathbb{R}} g_{n}(x, u)^{2} \mathrm{~d} u \stackrel{n \rightarrow \infty}{\longrightarrow} \int_{\mathbb{R}} f(x, u)^{2} \mathrm{~d} u, \quad \forall x
$$

Using

$$
f_{n}(x, u)=\frac{\sqrt{\sigma}}{2} n^{1 / 2-1 / 2 \alpha} \int_{0}^{1}\left(r_{n} h\right)^{T} \frac{\nabla_{\beta} p_{\frac{1}{n}}^{\beta+r_{n} h s}\left(x, \frac{u \sigma}{n^{1 / \alpha}}+\varsigma_{1}^{n, \theta, x}\right)}{p_{\frac{1}{n}}^{\beta+r_{n} h s}\left(x, \frac{u \sigma}{n^{1 / \alpha}}+\varsigma_{1}^{n, \theta, x}\right)^{1 / 2}} \mathrm{~d} s,
$$

we write:

$$
\begin{aligned}
& \int_{\mathbb{R}} f_{n}(x, u)^{2} \mathrm{~d} u=\left\|f_{n}(x, .)\right\|_{2}^{2}=\frac{\sigma n^{1-1 / \alpha}}{4}\left\|\int_{0}^{1}\left(r_{n} h\right)^{T} \frac{\nabla_{\beta} p_{\frac{1}{n}}^{\beta+r_{n} h s}\left(x, \sigma \frac{\dot{1}}{n^{1 / \alpha}}+\varsigma_{1}^{n, \theta, x}\right)}{p_{\frac{1}{n}}^{\beta+r_{n} h s}\left(x, \sigma \frac{\dot{n^{1 / \alpha}}}{n}+\varsigma_{1}^{n, \theta, x}\right)^{1 / 2}} \mathrm{~d} s\right\|_{2}^{2} \\
& \leq \frac{\sigma n^{1-1 / \alpha}}{4}\left(\int_{0}^{1}\left\|\left(r_{n} h\right)^{T} \frac{\nabla_{\beta} p_{\frac{1}{n}}^{\beta+r_{n} h s}\left(x, \sigma \frac{\dot{n^{1 / \alpha}}}{n}+\varsigma_{1}^{n, \theta, x}\right)}{p_{\frac{1}{n}}^{\beta+r_{n} h s}\left(x, \sigma \dot{\dot{n^{1 / \alpha}}}+\varsigma_{1}^{n, \theta, x}\right)^{1 / 2}}\right\|_{2} \mathrm{~d} s\right)^{2} \\
& =\frac{\sigma n^{1-1 / \alpha}}{4}\left(\int_{0}^{1}\left\|n^{\frac{1}{2}-\frac{1}{\alpha}} h_{1} \frac{\partial_{\theta} p_{\frac{1}{n}}^{\beta+r_{n} h s}\left(x, \sigma \frac{\dot{n^{1 / \alpha}}}{n^{\beta}}+\varsigma_{1}^{n, \theta, x}\right)}{p_{\frac{1}{n}}^{\beta+r_{n} h s}\left(x, \sigma \frac{\dot{n^{1 / \alpha}}}{n}+\varsigma_{1}^{n, \theta, x}\right)^{1 / 2}}+n^{\frac{-1}{2}} h_{2} \frac{\partial_{\sigma} p_{\frac{1}{n}}^{\beta+r_{n} h s}\left(x, \sigma \frac{\dot{n^{1 / \alpha}}}{2}+\varsigma_{1}^{n, \theta, x}\right)}{p_{\frac{1}{n}}^{\beta+r_{n} h s}\left(x, \sigma \frac{\dot{n^{1 / \alpha}}}{n}+\varsigma_{1}^{n, \theta, x}\right)^{1 / 2}}\right\|_{2} \mathrm{~d} s\right)^{2} \\
& =\frac{\sigma n^{1-1 / \alpha}}{4}\left[\int _ { 0 } ^ { 1 } \left(\int_{\mathbb{R}} n^{1-\frac{2}{\alpha}} h_{1}^{2} \frac{\partial_{\theta} p_{\frac{1}{n}}^{\beta+s r_{n} h}\left(x, \frac{u \sigma}{n^{1 / \alpha}}+\varsigma_{1}^{n, \theta, x}\right)^{2}}{p_{\frac{1}{n}}^{\beta+s r_{n} h}\left(x, \frac{u \sigma}{n^{1 / \alpha}}+\varsigma_{1}^{n, \theta, x}\right)} \mathrm{d} u+\int_{\mathbb{R}} n^{-1} h_{2}^{2} \frac{\partial_{\sigma} p_{\frac{1}{n}}^{\beta+s r_{n} h}\left(x, \frac{u \sigma}{n^{1 / \alpha}}+\varsigma_{1}^{n, \theta, x}\right)^{2}}{p_{\frac{1}{n}}^{\beta+s r_{n} h}\left(x, \frac{u \sigma}{n^{1 / \alpha}}+\varsigma_{1}^{n, \theta, x}\right)} \mathrm{d} u\right.\right. \\
& \left.\left.+\int_{\mathbb{R}} n^{-\frac{1}{\alpha}} 2 h_{1} h_{2} \frac{\partial_{\theta} p_{\frac{1}{n}}^{\beta+s r_{n} h}\left(x, \frac{u \sigma}{n^{1 / \alpha}}+\varsigma_{1}^{n, \theta, x}\right) \partial_{\sigma} p_{\frac{1}{n}}^{\beta+s r_{n} h}\left(x, \frac{u \sigma}{n^{1 / \alpha}}+\varsigma_{1}^{n, \theta, x}\right)}{p_{\frac{1}{n}}^{\beta+s r_{n} h}\left(x, \frac{u \sigma}{n^{1 / \alpha}}+\varsigma_{1}^{n, \theta, x}\right)} \mathrm{d} u\right)^{1 / 2} \mathrm{~d} s\right]^{2} \\
& =\frac{n^{1-1 / \alpha}}{4}\left[\int _ { 0 } ^ { 1 } \left(\int_{\mathbb{R}} n^{1-\frac{1}{\alpha}} h_{1}^{2} \frac{\partial_{\theta} p_{\frac{1}{n}}^{\beta+s r_{n} h}(x, v)^{2}}{p_{\frac{1}{n}}^{\beta+s r_{n} h}(x, v)} d v+\int_{\mathbb{R}} n^{\frac{1}{\alpha}-1} h_{2}^{2} \frac{\partial_{\sigma} p_{\frac{1}{n}}^{\beta+s r_{n} h}(x, v)^{2}}{p_{\frac{1}{n}}^{\beta+s r_{n} h}(x, v)} d v\right.\right. \\
& \left.\left.+\int_{\mathbb{R}} 2 h_{1} h_{2} \frac{\partial_{\theta} p_{\frac{1}{n}}^{\beta+s r_{n} h}(x, v) \partial_{\sigma} p_{\frac{1}{n}}^{n, \beta+s r_{n} h}(x, v)}{p_{\frac{1}{n}}^{\beta+s r_{n} h}(x, v)} d v\right)^{1 / 2} \mathrm{~d} s\right]^{2} \text { by the change of variable } \frac{u \sigma}{n^{1 / \alpha}}+\varsigma_{1}^{n, \theta, x}=v,
\end{aligned}
$$




$$
\begin{aligned}
& =\frac{1}{4}\left[\int_{0}^{1}\left(n^{2-\frac{2}{\alpha}} h_{1}^{2} \mathcal{I}_{11}^{n, \beta+s r_{n} h, x}+h_{2}^{2} \mathcal{I}_{22}^{n, \beta+s r_{n} h, x}+n^{1-1 / \alpha} 2 h_{1} h_{2} \mathcal{I}_{12}^{n, \beta+s r_{n} h, x}\right)^{1 / 2} \mathrm{~d} s\right]^{2} \\
& \stackrel{n \rightarrow \infty}{\longrightarrow} \frac{1}{\sigma^{2}} \frac{h_{1}^{2}}{4}\left(\partial_{\theta} b(x, \theta)\right)^{2} \int_{\mathbb{R}} \frac{\left(\varphi_{\alpha}^{\prime}(u)\right)^{2}}{\varphi_{\alpha}(u)} \mathrm{d} u+\frac{h_{1} h_{2}}{2 \sigma^{2}} \int_{\mathbb{R}} \frac{\varphi_{\alpha}^{\prime}(u)\left[\varphi_{\alpha}(u)+u \varphi_{\alpha}^{\prime}(u)\right]}{\varphi_{\alpha}(u)} \mathrm{d} u \\
& +\frac{1}{\sigma^{2}} \frac{h_{2}^{2}}{4} \int_{\mathbb{R}} \frac{\left(\varphi_{\alpha}(u)+u \varphi_{\alpha}^{\prime}(u)\right)^{2}}{\varphi_{\alpha}(u)} \mathrm{d} u=\int_{\mathbb{R}} f^{2}(x, u) \mathrm{d} u .
\end{aligned}
$$

Where, in the last line, we have used Theorem 3.1 for the convergences of $\mathcal{I}_{11}^{n, \beta+s r_{n} h, x}, \mathcal{I}_{22}^{n, \beta+s r_{n} h, x}, \mathcal{I}_{12}^{n, \beta+s r_{n} h, x}$, respectively and the application of the dominated convergence theorem. From (4.7) and (4.8) we get (4.4). (4.5) is deduced directly from Theorem 3.1.

\subsection{Proof of the conditions A2 and A3 (Thm. 2.4)}

From the Markov property and (3.1) we have:

$$
\mathcal{I}^{n, \beta, X_{i / n}^{\beta}}=\mathbb{E}\left[\xi_{i, n}(\beta) \xi_{i, n}(\beta)^{T} \mid \mathcal{G}_{i / n}\right]=\left(\begin{array}{ll}
\mathcal{I}_{11}^{n, \beta, X_{i / n}^{\beta}} & \mathcal{I}_{12}^{n, \beta, X_{i / n}^{\beta}} \\
\mathcal{I}_{12}^{n, \beta, X_{i / n}^{\beta}} & \mathcal{I}_{22}^{n, \beta, X_{i / n}^{\beta}}
\end{array}\right)
$$

From Theorem 3.1 we know that the quantities

$$
\sup _{0 \leq i \leq n-1}\left|n r_{n} \mathcal{I}^{n, \beta, X_{i / n}^{\beta}} r_{n}-\left(\begin{array}{cc}
\frac{1}{\sigma^{2}}\left[\partial_{\theta} b\left(X_{i / n}^{\beta}\right)\right]^{2} \int_{\mathbb{R}} \frac{\varphi^{\prime}(u)^{2}}{\varphi(u)} \mathrm{d} u & 0 \\
0 & \frac{1}{\sigma^{2}} \int_{\mathbb{R}} \frac{\left[\varphi_{\alpha}(u)+u \varphi_{\alpha}^{\prime}(u)\right]^{2}}{\varphi_{\alpha}(u)} \mathrm{d} u
\end{array}\right)\right|
$$

converge to zero as $n \rightarrow \infty$. Then the convergence $\mathbf{A 2}$ is a consequence of the convergence of a Riemann sum.

To prove A3, from the Markov property, we get: $\mathbb{E}\left[\left|r_{n} \xi_{i, n}(\beta)\right|^{k} \mid X_{i / n}^{\beta}=x\right]=\mathbb{E}\left[\left|r_{n} \frac{\nabla_{\beta} p_{\frac{1}{n}}^{\beta}\left(x, X_{\frac{1}{n}}^{\beta}\right)}{p_{\frac{1}{n}}^{\beta}\left(x, X_{\frac{1}{n}}^{\beta}\right)}\right|^{k}\right]$, for any $k \geq 1$. But from Theorem $3.1 \mathrm{ii})$ we have $\sup _{n, x} \mathbb{E}\left[\left|n^{1 / 2} r_{n} \frac{\nabla_{\beta} p_{\frac{1}{n}}^{\beta}\left(x, X_{\frac{1}{n}}^{\beta}\right)}{p_{\frac{1}{n}}^{\beta}\left(x, X_{\frac{1}{n}}^{\beta}\right)}\right|^{k}\right]<\infty, \forall k \geq 1$. This control, for instance with $k=4$, is sufficient to imply the Lindeberg's condition A3.

\section{Proof of Theorem 2.5 (Stable Central limit theorem)}

The aim of this section is to prove the stable convergence in law stated in Theorem 2.5. We first recall the following result established in [6] where $\bar{Y}_{1}^{n, \beta, x_{0}}$ is defined by (3.4) and is equal in law to $X_{\frac{1}{n}}^{\beta}$.

Lemma 5.1 (Lem. 4.1 in [6]). Let $\left(\varsigma_{t}^{n, \theta, x_{0}}\right)$ be the solution to the ordinary differential equation (4.1), then

$$
n^{1 / \alpha}\left(\bar{Y}_{1}^{n, \beta, x_{0}}-\varsigma_{1}^{n, \theta, x_{0}}\right) \underset{\text { a.s. }}{\stackrel{n \rightarrow \infty}{\longrightarrow}} \sigma L_{1}^{\alpha},
$$

and this convergence is uniform with respect to $x_{0}$. 
Proof of Theorem 2.5. Since $r_{n}=\left(\begin{array}{cc}n^{\frac{1}{2}-\frac{1}{\alpha}} & 0 \\ 0 & n^{-\frac{1}{2}}\end{array}\right)$ we have

$$
r_{n} \sum_{i=0}^{n-1} \xi_{i, n}(\beta)=\sum_{i=0}^{n-1}\left(\begin{array}{c}
n^{\frac{1}{2}-\frac{1}{\alpha}} \frac{\partial_{\theta} p_{\frac{1}{n}}^{\beta}}{p_{\frac{1}{n}}^{\beta}}\left(X_{\frac{i}{n}}^{\beta}, X_{\frac{i+1}{n}}^{\beta}\right) \\
n^{-\frac{1}{2}} \frac{\partial_{\sigma} p_{\frac{1}{n}}^{\beta}}{p_{\frac{1}{n}}^{\beta}}\left(X_{\frac{i}{n}}^{\beta}, X_{\frac{i+1}{n}}^{\beta}\right)
\end{array}\right) .
$$

Theorem 2.5 is an immediate consequence of Lemmas 5.2-5.4 below.

Lemma 5.2. We consider

$$
\varpi_{i, n}=n^{1-1 / \alpha} \frac{\partial_{\theta} p_{\frac{1}{n}}^{\beta}}{p_{\frac{1}{n}}^{\beta}}\left(X_{\frac{i}{n}}^{\beta}, X_{\frac{i+1}{n}}^{\beta}\right)+\frac{1}{\sigma} \partial_{\theta} b\left(X_{\frac{i}{n}}^{\beta}, \theta\right) \frac{\varphi_{\alpha}^{\prime}\left(n^{1 / \alpha}\left(L_{\frac{i+1}{n}}-L_{\frac{i}{n}}\right)\right)}{\varphi_{\alpha}\left(n^{1 / \alpha}\left(L_{\frac{i+1}{n}}-L_{\frac{i}{n}}\right)\right)}
$$

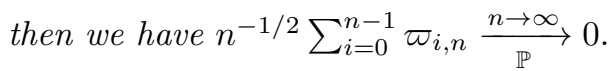

Proof. Using Lemma 9 in [7], it is sufficient to show that:

$$
\begin{array}{r}
n^{-1 / 2} \sum_{i=0}^{n-1}\left|\mathbb{E}\left[\varpi_{i, n} \mid \mathcal{F}_{i / n}\right]\right| \underset{\mathbb{P}}{\stackrel{n \rightarrow \infty}{\longrightarrow}} 0, \\
n^{-1} \sum_{i=0}^{n-1}\left|\mathbb{E}\left[\varpi_{i, n}^{2} \mid \mathcal{F}_{i / n}\right]\right| \stackrel{n \rightarrow \infty}{\underset{P}{\longrightarrow}} 0,
\end{array}
$$

We start by the proof of (5.2). Since a score function has an expectation equal to zero, and $L_{\frac{i+1}{n}}-L_{\frac{i}{n}}$ is independent of $\mathcal{F}_{i / n}$, we deduce that

$$
\mathbb{E}\left[\varpi_{i, n} \mid \mathcal{F}_{i / n}\right]=\frac{1}{\sigma} \partial_{\theta} b\left(X_{\frac{i}{n}}^{\beta}, \theta\right) \mathbb{E}\left[\frac{\varphi_{\alpha}^{\prime}\left(n^{1 / \alpha}\left(L_{\frac{i+1}{n}}-L_{\frac{i}{n}}\right)\right)}{\varphi_{\alpha}\left(n^{1 / \alpha}\left(L_{\frac{i+1}{n}}-L_{\frac{i}{n}}\right)\right)}\right] .
$$

Since $\left(L_{t}\right)_{t}$ has stationary increments, the law of $n^{1 / \alpha}\left(L_{\frac{i+1}{n}}-L_{\frac{i}{n}}\right)$ is the same as the law of $L_{1}^{n}$. Moreover, we know from Lemma 3.3 , that $\mathbb{P}\left(L_{1}^{n} \neq L_{1}^{\alpha}\right)=O(1 / n)$, thus

$$
\mathbb{E}\left[\varpi_{i, n} \mid \mathcal{F}_{i / n}\right]=\frac{1}{\sigma} \partial_{\theta} b\left(X_{\frac{i}{n}}^{\beta}, \theta\right) \mathbb{E}\left[\frac{\varphi_{\alpha}^{\prime}\left(L_{1}^{\alpha}\right)}{\varphi_{\alpha}\left(L_{1}^{\alpha}\right)}\right]+\left\|\frac{\varphi_{\alpha}^{\prime}}{\varphi_{\alpha}}\right\|_{\infty} O\left(n^{-1}\right)
$$

where we used the fact that $\frac{\varphi_{\alpha}^{\prime}}{\varphi_{\alpha}}$ is bounded (see e.g. Thm. 7.3.2 in [19]). Using $\mathbb{E}\left[\frac{\varphi_{\alpha}^{\prime}\left(L_{1}^{\alpha}\right)}{\varphi_{\alpha}\left(L_{1}^{\alpha}\right)}\right]=\int_{\mathbb{R}} \varphi_{\alpha}^{\prime}(u) \mathrm{d} u=0$, we deduce $\left|\mathbb{E}\left[\varpi_{i, n} \mid \mathcal{G}_{i / n}\right]\right| \leq C n^{-1}$ for some constant $C$ and (5.2) follows. 
We now prove (5.3). We have

$$
\begin{aligned}
\mathbb{E}\left[\varpi_{i, n}^{2} \mid \mathcal{F}_{i / n}\right]= & n^{2-2 / \alpha} \mathcal{I}_{11}^{n, \beta, X_{\frac{i}{n}}^{\beta}}+\frac{1}{\sigma^{2}}\left[\partial_{\theta} b\left(X_{\frac{i}{n}}^{\beta}, \theta\right)\right]^{2} \mathbb{E}\left[\frac{\varphi_{\alpha}^{\prime}\left(n^{1 / \alpha}\left(L_{\frac{i+1}{n}}-L_{\frac{i}{n}}\right)\right)^{2}}{\varphi_{\alpha}\left(n^{1 / \alpha}\left(L_{\frac{i+1}{n}}-L_{\frac{i}{n}}\right)\right)^{2}}\right] \\
& +2 \mathbb{E}\left[n^{1-1 / \alpha} \frac{\partial_{\theta} p_{\frac{1}{n}}^{\beta}\left(X_{\frac{i}{n}}^{\beta}, X_{\frac{i+1}{n}}^{\beta}\right)}{p_{\frac{1}{n}}^{\beta}\left(X_{\frac{i}{n}}^{\beta}, X_{\frac{i+1}{n}}^{\beta}\right)} \frac{1}{\sigma} \partial_{\theta} b\left(X_{\frac{i}{n}}^{\beta}, \theta\right) \frac{\varphi_{\alpha}^{\prime}\left(n^{1 / \alpha}\left(L_{\frac{i+1}{n}}-L_{\frac{i}{n}}\right)\right)}{\varphi_{\alpha}\left(n^{1 / \alpha}\left(L_{\frac{i+1}{n}}-L_{\frac{i}{n}}\right)\right)} \mid \mathcal{F}_{\frac{i}{n}}\right] .
\end{aligned}
$$

With a method analogous to the proof of (5.2), we can show that

$\mathbb{E}\left[\frac{\varphi_{\alpha}^{\prime}\left(n^{1 / \alpha}\left(L_{\frac{i+1}{n}}-L_{\frac{i}{n}}\right)\right)^{2}}{\varphi_{\alpha}\left(n^{1 / \alpha}\left(L_{\frac{i+1}{n}}-L_{\frac{i}{n}}\right)\right)^{2}}\right]=\mathbb{E}\left[\frac{\varphi_{\alpha}^{\prime}\left(L_{1}^{\alpha}\right)^{2}}{\varphi_{\alpha}\left(L_{1}^{\alpha}\right)^{2}}\right]+o(1)$. From Theorem 3.1, it appears that the first two terms in the right-hand side of (5.4) are asymptotically close to the same quantities, and that (5.3) is proved as soon as we show that the following control holds, uniformly with respect to $i$,

$\mathbb{E}\left[n^{1-1 / \alpha} \frac{\partial_{\theta} p_{\frac{1}{n}}^{\beta}\left(X_{\frac{i}{n}}^{\beta}, X_{\frac{i+1}{n}}^{\beta}\right)}{p_{\frac{1}{n}}^{\beta}\left(X_{\frac{i}{n}}^{\beta}, X_{\frac{i+1}{n}}^{\beta}\right)} \frac{1}{\sigma} \partial_{\theta} b\left(X_{\frac{i}{n}}^{\beta}, \theta\right) \frac{\varphi_{\alpha}^{\prime}\left(n^{1 / \alpha}\left(L_{\frac{i+1}{n}}-L_{\frac{i}{n}}\right)\right)}{\varphi_{\alpha}\left(n^{1 / \alpha}\left(L_{\frac{i+1}{n}}-L_{\frac{i}{n}}\right)\right)} \mid \mathcal{F}_{\frac{i}{n}}\right]=-\frac{1}{\sigma^{2}}\left[\partial_{\theta} b\left(X_{\frac{i}{n}}^{\beta}, \theta\right)\right]^{2} \mathbb{E}\left[\frac{\varphi_{\alpha}^{\prime}\left(L_{1}^{\alpha}\right)^{2}}{\varphi_{\alpha}\left(L_{1}^{\alpha}\right)^{2}}\right]+o(1)$.

Using the notations of Section 3.2.1, we define $d^{n, \theta, x_{0}}=\mathbb{E}\left[n^{1-1 / \alpha} \frac{\partial_{\theta} q^{n, \beta, x_{0}}\left(\bar{Y}_{1}^{n, \beta, x_{0}}\right)}{q^{n, \beta, x_{0}}\left(\bar{Y}_{1}^{n, \beta, x_{0}}\right)} \frac{1}{\sigma} \partial_{\theta} b\left(x_{0}, \theta\right) \frac{\varphi_{\alpha}^{\prime}\left(L_{1}^{n}\right)}{\varphi_{\alpha}\left(L_{1}^{n}\right)}\right]$, so that the left-hand side of (5.5) reduces, from the Markov property, (3.5) and the fact that $\bar{Y}_{1}^{n, \beta, x_{0}}$ equals in law $X_{\frac{1}{n}}^{\beta}$, to $d^{n, \theta, X_{i / n}^{\beta}}$. On the other hand, we can rewrite $d^{n, \theta, x_{0}}$ as

$$
\begin{aligned}
& d^{n, \theta, x_{0}}=\mathbb{E}\left[n^{1-1 / \alpha} \frac{\partial_{\theta} q^{n, \beta, x_{0}}\left(\bar{Y}_{1}^{n, \beta, x_{0}}\right)}{q^{n, \beta, x_{0}}\left(\bar{Y}_{1}^{n, \beta, x_{0}}\right)} \frac{1}{\sigma} \partial_{\theta} b\left(x_{0}, \theta\right) \frac{\varphi_{\alpha}^{\prime}\left(\frac{n^{1 / \alpha}\left(\bar{Y}_{1}^{n, \beta, x_{0}}-\varsigma_{1}^{n, \theta, x_{0}}\right)}{\sigma}\right)}{\varphi_{\alpha}\left(\frac{n^{1 / \alpha}\left(\bar{Y}_{1}^{n, \beta, x_{0}}-\varsigma_{1}^{n, \theta, x_{0}}\right)}{\sigma}\right)}\right] \\
& +\mathbb{E}\left[n^{1-1 / \alpha} \frac{\partial_{\theta} q^{n, \beta, x_{0}}\left(\bar{Y}_{1}^{n, \beta, x_{0}}\right)}{q^{n, \beta, x_{0}}\left(\bar{Y}_{1}^{n, \beta, x_{0}}\right)} \frac{1}{\sigma} \partial_{\theta} b\left(x_{0}, \theta\right)\left(\frac{\varphi_{\alpha}^{\prime}\left(L_{1}^{n}\right)}{\varphi_{\alpha}\left(L_{1}^{n}\right)}-\frac{\varphi_{\alpha}^{\prime}\left(\frac{n^{1 / \alpha}\left(\bar{Y}_{1}^{n, \beta, x_{0}}-\varsigma_{1}^{n, \theta, x_{0}}\right)}{\sigma}\right)}{\varphi_{\alpha}\left(\frac{n^{1 / \alpha}\left(\bar{Y}_{1}^{n, \beta, x_{0}}-\varsigma_{1}^{n, \theta, x_{0}}\right)}{\sigma}\right)}\right)\right] .
\end{aligned}
$$

Using the Cauchy-Schwarz inequality for the second term in the right-hand side of (5.6), we get

$$
\begin{aligned}
& \left.\mathbb{E}\left[\mid n^{1-1 / \alpha} \frac{\partial_{\theta} q^{n, \beta, x_{0}}\left(\bar{Y}_{1}^{n, \beta, x_{0}}\right)}{q^{n, \beta, x_{0}}\left(\bar{Y}_{1}^{n, \beta, x_{0}}\right)} \frac{1}{\sigma} \partial_{\theta} b\left(x_{0}, \theta\right)\left(\frac{\varphi_{\alpha}^{\prime}\left(L_{1}^{n}\right)}{\varphi_{\alpha}\left(L_{1}^{n}\right)}-\frac{\varphi_{\alpha}^{\prime}\left(\frac{n^{1 / \alpha}\left(\bar{Y}_{1}^{n, \beta, x_{0}}-\varsigma_{1}^{n, \theta, x_{0}}\right)}{\sigma}\right)}{\varphi_{\alpha}\left(\frac{n^{1 / \alpha}\left(\bar{Y}_{1}^{n, \beta, x_{0}}-\varsigma_{1}^{n, \theta, x_{0}}\right)}{\sigma}\right)}\right)\right]\right] \\
& \leq\left[\mathbb{E}\left(n^{1-1 / \alpha} \frac{\partial_{\theta} q^{n, \beta, x_{0}}\left(\bar{Y}_{1}^{n, \beta, x_{0}}\right)}{q^{n, \beta, x_{0}}\left(\bar{Y}_{1}^{n, \beta, x_{0}}\right)} \frac{1}{\sigma} \partial_{\theta} b\left(x_{0}, \theta\right)\right)^{2}\right]^{1 / 2}\left[\mathbb{E}\left(\frac{\varphi_{\alpha}^{\prime}\left(L_{1}^{n}\right)}{\varphi_{\alpha}\left(L_{1}^{n}\right)}-\frac{\varphi_{\alpha}^{\prime}\left(\frac{n^{1 / \alpha}\left(\bar{Y}_{1}^{n, \beta, x_{0}}-\varsigma_{1}^{n, \theta, x_{0}}\right)}{\sigma}\right)}{\varphi_{\alpha}\left(\frac{n^{1 / \alpha}\left(\bar{Y}_{1}^{n, \beta, x_{0}}-\varsigma_{1}^{n, \theta, x_{0}}\right)}{\sigma}\right)}\right)^{2}\right]^{1 / 2} .
\end{aligned}
$$


Furthermore, $\forall \epsilon>0$ we have

$$
\begin{aligned}
& \mathbb{E}\left(\frac{\varphi_{\alpha}^{\prime}\left(L_{1}^{n}\right)}{\varphi_{\alpha}\left(L_{1}^{n}\right)}-\frac{\varphi_{\alpha}^{\prime}\left(\frac{n^{1 / \alpha}\left(\bar{Y}_{1}^{n, \beta, x_{0}}-\varsigma_{1}^{n, \theta, x_{0}}\right)}{\sigma}\right)}{\varphi_{\alpha}\left(\frac{n^{1 / \alpha}\left(\bar{Y}_{1}^{n, \beta, x_{0}}-\varsigma_{1}^{n, \theta, x_{0}}\right)}{\sigma}\right)}\right)^{2} \\
= & \mathbb{E}\left(\frac{\varphi_{\alpha}^{\prime}\left(L_{1}^{n}\right)}{\varphi_{\alpha}\left(L_{1}^{n}\right)}-\frac{\varphi_{\alpha}^{\prime}\left(\frac{n^{1 / \alpha}\left(\bar{Y}_{1}^{n, \beta, x_{0}}-\varsigma_{1}^{n, \theta, x_{0}}\right)}{\sigma}\right)}{\varphi_{\alpha}\left(\frac{n^{1 / \alpha}\left(\bar{Y}_{1}^{n, \beta, x_{0}}-\varsigma_{1}^{n, \theta, x_{0}}\right)}{\sigma}\right)}\right)^{2}\left\{\left|\frac{n^{1 / \alpha}\left(\bar{Y}_{1}^{n, \theta, x_{0}}-\varsigma_{1}^{n, \theta, x_{0}}\right)}{\sigma}-L_{1}^{n}\right| \leq \epsilon\right\} \\
& +\mathbb{E}\left(\frac{\varphi_{\alpha}^{\prime}\left(L_{1}^{n}\right)}{\varphi_{\alpha}\left(L_{1}^{n}\right)}-\frac{\varphi_{\alpha}^{\prime}\left(\frac{n^{1 / \alpha}\left(\bar{Y}_{1}^{n, \beta, x_{0}}-\varsigma_{1}^{n, \theta, x_{0}}\right)}{\sigma}\right)}{\varphi_{\alpha}\left(\frac{n^{1 / \alpha}\left(\bar{Y}_{1}^{n, \beta, x_{0}}-\varsigma_{1}^{n, \theta, x_{0}}\right)}{\sigma}\right)}\right)^{2}\left\{\left|\frac{n^{1 / \alpha}\left(\bar{Y}_{1}^{n, \beta, x_{0}}-\varsigma_{1}^{n, \theta, x_{0}}\right)}{\sigma}-L_{1}^{n}\right|>\epsilon\right\} \\
\leq & \left.C_{1} \epsilon^{2}+2 C_{2} \mathbb{E}\left[1|| \frac{n^{1 / \alpha}\left(\bar{Y}_{1}^{n, \beta, x_{0}}-\varsigma_{1}^{n, \theta, x_{0}}\right)}{\sigma}-L_{1}^{n} \mid>\epsilon\right\}\right] \\
= & C_{1} \epsilon^{2}+2 C_{2} \mathbb{P}\left[\left|\frac{n^{1 / \alpha}\left(\bar{Y}_{1}^{n, \beta, x_{0}}-\varsigma_{1}^{n, \theta, x_{0}}\right)}{\sigma}-L_{1}^{n}\right|>\epsilon\right] \frac{n \rightarrow \infty}{\longrightarrow} C_{1} \epsilon^{2},
\end{aligned}
$$

where we used the fact that $\frac{\varphi_{\alpha}^{\prime}}{\varphi_{\alpha}}$ is bounded with a bounded derivative (see e.g. Thm. 7.3.2 in [19]) and Lemma 5.1. From Theorem $3.1 \mathrm{ii}$ ), and the estimates above we deduce that (5.7) converges to zero as $n \rightarrow \infty$. Then,

$$
d^{n, \theta, x_{0}}=\mathbb{E}\left[n^{1-1 / \alpha} \frac{\partial_{\theta} q^{n, \beta, x_{0}}\left(\bar{Y}_{1}^{n, \beta, x_{0}}\right)}{q^{n, \beta, x_{0}}\left(\bar{Y}_{1}^{n, \beta, x_{0}}\right)} \frac{1}{\sigma} \partial_{\theta} b\left(x_{0}, \theta\right) \frac{\varphi_{\alpha}^{\prime}\left(\frac{n^{1 / \alpha}\left(\bar{Y}_{1}^{n, \beta, x_{0}}-\varsigma_{1}^{n, \theta, x_{0}}\right)}{\sigma}\right)}{\varphi_{\alpha}\left(\frac{n^{1 / \alpha}\left(\bar{Y}_{1}^{n, \theta, x_{0}}-\varsigma_{1}^{n, \theta, x_{0}}\right)}{\sigma}\right)}\right]+o(1),
$$

where the $o(1)$ term is uniform with respect to $x_{0}$. Now, using Proposition 3.4, we get

$$
d^{n, \theta, x_{0}}=\mathbb{E}\left[n^{1-1 / \alpha} \mathcal{H}_{\bar{Y}_{1}^{n, \beta, x_{0}}}\left(\partial_{\theta} \bar{Y}_{1}^{n, \beta, x_{0}}\right) \frac{1}{\sigma} \partial_{\theta} b\left(x_{0}, \theta\right) \frac{\varphi_{\alpha}^{\prime}\left(\frac{n^{1 / \alpha}\left(\bar{Y}_{1}^{n, \beta, x_{0}}-\varsigma_{1}^{n, \theta, x_{0}}\right)}{\sigma}\right)}{\varphi_{\alpha}\left(\frac{n^{1 / \alpha}\left(\bar{Y}_{1}^{n, \beta, x_{0}}-\varsigma_{1}^{n, \theta, x_{0}}\right)}{\sigma}\right)}\right]+o(1) .
$$

From Lemma 3.6, we also have

$$
\sup _{x_{0}}\left|d^{n, \theta, x_{0}}-\frac{1}{\sigma^{2}} \partial_{\theta} b\left(x_{0}, \theta\right)^{2} \mathbb{E}\left[\mathcal{H}_{L^{\alpha}}(1) \frac{\varphi_{\alpha}^{\prime}\left(\frac{n^{1 / \alpha}\left(\bar{Y}_{1}^{n, \beta, x_{0}}-\varsigma_{1}^{n, \theta, x_{0}}\right)}{\sigma}\right)}{\varphi_{\alpha}\left(\frac{n^{1 / \alpha}\left(\bar{Y}_{1}^{n, \beta, x_{0}}-\varsigma_{1}^{n, \theta, x_{0}}\right)}{\sigma}\right)}\right]\right| \stackrel{n \rightarrow \infty}{\longrightarrow} 0 .
$$

From Lemma 5.1 we can deduce that

$$
d^{n, \theta, x_{0}} \stackrel{n \rightarrow \infty}{\longrightarrow} \frac{1}{\sigma^{2}}\left[\partial_{\theta} b\left(x_{0}, \theta\right)\right]^{2} \mathbb{E}\left[\mathcal{H}_{L^{\alpha}}(1) \frac{\varphi_{\alpha}^{\prime}\left(L_{1}^{\alpha}\right)}{\varphi_{\alpha}\left(L_{1}^{\alpha}\right)}\right]
$$


uniformly with respect to $x_{0}$. Then, the relation (3.7) enables to rewrite this convergence as,

$$
d^{n, \theta, x_{0}} \stackrel{n \rightarrow \infty}{\longrightarrow}-\frac{1}{\sigma^{2}}\left[\partial_{\theta} b\left(x_{0}, \theta\right)\right]^{2} \mathbb{E}\left[\frac{\varphi_{\alpha}^{\prime}\left(L_{1}^{\alpha}\right)^{2}}{\varphi_{\alpha}\left(L_{1}^{\alpha}\right)^{2}}\right] \text {, uniformly with respect to } x_{0} .
$$

This result implies (5.5) and hence (5.3).

Lemma 5.3. We consider

$$
\varrho_{i, n}=\frac{\partial_{\sigma} p_{\frac{1}{n}}^{\beta}}{p_{\frac{1}{n}}^{\beta}}\left(X_{\frac{i}{n}}^{\beta}, X_{\frac{i+1}{n}}^{\beta}\right)+\frac{1}{\sigma} \frac{\varphi_{\alpha}\left(n^{1 / \alpha}\left(L_{\frac{i+1}{n}}-L_{\frac{i}{n}}\right)\right)+n^{1 / \alpha}\left(L_{\frac{i+1}{n}}-L_{\frac{i}{n}}\right) \varphi_{\alpha}^{\prime}\left(n^{1 / \alpha}\left(L_{\frac{i+1}{n}}-L_{\frac{i}{n}}\right)\right)}{\varphi_{\alpha}\left(n^{1 / \alpha}\left(L_{\frac{i+1}{n}}-L_{\frac{i}{n}}\right)\right)}
$$

then we have $n^{-1 / 2} \sum_{i=0}^{n-1} \varrho_{i, n} \underset{\mathbb{P}}{\stackrel{n \rightarrow \infty}{\longrightarrow}} 0$.

Proof. We proceed as in the proof of Lemma 5.2 and check that

$$
\begin{array}{r}
n^{-1 / 2} \sum_{i=0}^{n-1}\left|\mathbb{E}\left[\varrho_{i, n} \mid \mathcal{F}_{i / n}\right]\right| \stackrel{n \rightarrow \infty}{\mathbb{P}} 0, \\
n^{-1} \sum_{i=0}^{n-1}\left|\mathbb{E}\left[\varrho_{i, n}^{2} \mid \mathcal{F}_{i / n}\right]\right| \underset{\mathbb{P}}{\stackrel{n \rightarrow \infty}{\longrightarrow}} 0 .
\end{array}
$$

We start by the proof of (5.9). Since a score function has an expectation equal to zero, and $L_{\frac{i+1}{n}}-L_{\frac{i}{n}}$ is independent of $\mathcal{F}_{i / n}$, we deduce that

$$
\mathbb{E}\left[\varrho_{i, n} \mid \mathcal{F}_{i / n}\right]=\frac{1}{\sigma} \mathbb{E}\left[\frac{\varphi_{\alpha}\left(n^{1 / \alpha}\left(L_{\frac{i+1}{n}}-L_{\frac{i}{n}}\right)\right)+n^{1 / \alpha}\left(L_{\frac{i+1}{n}}-L_{\frac{i}{n}}\right) \varphi_{\alpha}^{\prime}\left(n^{1 / \alpha}\left(L_{\frac{i+1}{n}}-L_{\frac{i}{n}}\right)\right)}{\varphi_{\alpha}\left(n^{1 / \alpha}\left(L_{\frac{i+1}{n}}-L_{\frac{i}{n}}\right)\right)}\right] .
$$

Since $\left(L_{t}\right)_{t}$ has stationary increments, the law of $n^{1 / \alpha}\left(L_{\frac{i+1}{n}}-L_{\frac{i}{n}}\right)$ is the same as the law of $L_{1}^{n}$. Moreover, we know from Lemma 3.3 , that $\mathbb{P}\left(L_{1}^{n} \neq L_{1}^{\alpha}\right)=O(1 / n)$, thus

$$
\mathbb{E}\left[\varrho_{i, n} \mid \mathcal{F}_{i / n}\right]=\frac{1}{\sigma} \mathbb{E}\left[\frac{\varphi_{\alpha}\left(L_{1}^{\alpha}\right)+L_{1}^{\alpha} \varphi_{\alpha}^{\prime}\left(L_{1}^{\alpha}\right)}{\varphi_{\alpha}\left(L_{1}^{\alpha}\right)}\right]+\left\|\frac{\varphi_{\alpha}(u)+u \varphi_{\alpha}^{\prime}(u)}{\varphi_{\alpha}(u)}\right\|_{\infty} O\left(n^{-1}\right),
$$

where we used the fact that $\frac{u \varphi_{\alpha}^{\prime}(u)}{\varphi_{\alpha}(u)}$ is bounded (see e.g. Thm. 7.3.2 in [19]). Using $\mathbb{E}\left[\frac{L_{1}^{\alpha} \varphi_{\alpha}^{\prime}\left(L_{1}^{\alpha}\right)}{\varphi_{\alpha}\left(L_{1}^{\alpha}\right)}\right]=\int_{\mathbb{R}} u \varphi_{\alpha}^{\prime}(u) \mathrm{d} u=$ -1 , we deduce $\left|\mathbb{E}\left[\varrho_{i, n} \mid \mathcal{F}_{i / n}\right]\right| \leq C n^{-1}$ for some constant $C$ and (5.9) follows.

We now prove (5.10). We have

$$
\begin{aligned}
\mathbb{E}\left[\varrho_{i, n}^{2} \mid \mathcal{F}_{i / n}\right]= & \mathcal{I}_{22}^{n, \beta, X_{\frac{i}{n}}^{\beta}}+\frac{1}{\sigma^{2}} \mathbb{E}\left[\frac{\left[\varphi_{\alpha}\left(n^{1 / \alpha}\left(L_{\frac{i+1}{n}}-L_{\frac{i}{n}}\right)\right)+n^{1 / \alpha}\left(L_{\frac{i+1}{n}}-L_{\frac{i}{n}}\right) \varphi_{\alpha}^{\prime}\left(n^{1 / \alpha}\left(L_{\frac{i+1}{n}}-L_{\frac{i}{n}}\right)\right)\right]^{2}}{\varphi_{\alpha}\left(n^{1 / \alpha}\left(L_{\frac{i+1}{n}}-L_{\frac{i}{n}}\right)\right)^{2}}\right] \\
& +2 \mathbb{E}\left[\frac{\partial_{\sigma} p_{\frac{1}{n}}^{\beta}\left(X_{\frac{i}{n}}^{\beta}, X_{\frac{i+1}{n}}^{\beta}\right)}{p_{\frac{1}{n}}^{\beta}\left(X_{\frac{i}{n}}^{\beta}, X_{\frac{i+1}{n}}^{\beta}\right)} \frac{\varphi_{\alpha}\left(n^{1 / \alpha}\left(L_{\frac{i+1}{n}}-L_{\frac{i}{n}}\right)\right)+n^{1 / \alpha}\left(L_{\frac{i+1}{n}}-L_{\frac{i}{n}}\right) \varphi_{\alpha}^{\prime}\left(n^{1 / \alpha}\left(L_{\frac{i+1}{n}}-L_{\frac{i}{n}}\right)\right)}{\varphi_{\alpha}\left(n^{1 / \alpha}\left(L_{\frac{i+1}{n}}-L_{\frac{i}{n}}\right)\right)} \mid \mathcal{F}_{\frac{i}{n}}\right]
\end{aligned}
$$


With a method analogous to the proof of (5.9), we can show that

$$
\mathbb{E}\left[\frac{\left[\varphi_{\alpha}\left(n^{1 / \alpha}\left(L_{\frac{i+1}{n}}-L_{\frac{i}{n}}\right)\right)+n^{1 / \alpha}\left(L_{\frac{i+1}{n}}-L_{\frac{i}{n}}\right) \varphi_{\alpha}^{\prime}\left(n^{1 / \alpha}\left(L_{\frac{i+1}{n}}-L_{\frac{i}{n}}\right)\right)\right]^{2}}{\varphi_{\alpha}\left(n^{1 / \alpha}\left(L_{\frac{i+1}{n}}-L_{\frac{i}{n}}\right)\right)^{2}}\right]=\mathbb{E}\left[\frac{\left[\varphi_{\alpha}\left(L_{1}^{\alpha}\right)+L_{1}^{\alpha} \varphi_{\alpha}^{\prime}\left(L_{1}^{\alpha}\right)\right]^{2}}{\varphi_{\alpha}\left(L_{1}^{\alpha}\right)^{2}}\right]+o(1) .
$$

Proceeding as in the proof of (5.4), then (5.9) is proved as soon as we show that the following control holds uniformly with respect to $i$,

$$
\begin{aligned}
& \mathbb{E}\left[\frac{\partial_{\sigma} p_{\frac{1}{n}}^{\beta}\left(X_{\frac{i}{n}}^{\beta}, X_{\frac{i+1}{n}}^{\beta}\right)}{p_{\frac{1}{n}}^{\beta}\left(X_{\frac{i}{n}}^{\beta}, X_{\frac{i+1}{n}}^{\beta}\right)} \frac{\varphi_{\alpha}\left(n^{1 / \alpha}\left(L_{\frac{i+1}{n}}-L_{\frac{i}{n}}\right)\right)+n^{1 / \alpha}\left(L_{\frac{i+1}{n}}-L_{\frac{i}{n}}\right) \varphi_{\alpha}^{\prime}\left(n^{1 / \alpha}\left(L_{\frac{i+1}{n}}-L_{\frac{i}{n}}\right)\right)}{\varphi_{\alpha}\left(n^{1 / \alpha}\left(L_{\frac{i+1}{n}}-L_{\frac{i}{n}}\right)\right)} \mid \mathcal{F}_{\frac{i}{n}}\right] \\
& =-\frac{1}{\sigma^{2}} \mathbb{E}\left[\frac{\left(\varphi_{\alpha}\left(L_{1}^{\alpha}\right)+L_{1}^{\alpha} \varphi_{\alpha}^{\prime}\left(L_{1}^{\alpha}\right)\right)^{2}}{\varphi_{\alpha}\left(L_{1}^{\alpha}\right)^{2}}\right]+o(1) .
\end{aligned}
$$

We define $d_{1}^{n, \sigma, x_{0}}=\mathbb{E}\left[\frac{\partial_{\sigma} q\left(\bar{Y}_{1}^{n, \beta, x_{0}}\right)}{q\left(\bar{Y}_{1}^{n, \beta, x_{0}}\right)} \frac{1}{\sigma} \frac{\varphi_{\alpha}\left(L_{1}^{n}\right)+L_{1}^{n} \varphi_{\alpha}^{\prime}\left(L_{1}^{n}\right)}{\varphi_{\alpha}\left(L_{1}^{n}\right)}\right]$, so that the left-hand side of (5.12) reduces, from the Markov property, to $d_{1}^{n, \sigma, X_{i / n}^{\beta}}$.

Proceeding as in the proof of (5.8), noting that $\frac{u \varphi_{\alpha}^{\prime}(u)}{\varphi_{\alpha}(u)}$ is bounded with a bounded derivative (see e.g. Thm. 7.3.2 in [19]), then we also get that

$$
d_{1}^{n, \sigma, x_{0}}=\mathbb{E}\left[\frac{\partial_{\sigma} q\left(\bar{Y}_{1}^{n, \beta, x_{0}}\right)}{q\left(\bar{Y}_{1}^{n, \beta, x_{0}}\right)} \frac{1}{\sigma}\right]+\mathbb{E}\left[\frac{\partial_{\sigma} q\left(\bar{Y}_{1}^{n, \beta, x_{0}}\right)}{q\left(\bar{Y}_{1}^{n, \beta, x_{0}}\right)} \frac{1}{\sigma} \frac{\frac{n^{1 / \alpha}\left(\bar{Y}_{1}^{n, \beta, x_{0}}-\varsigma_{1}^{n, \theta, x_{0}}\right)}{\sigma} \varphi_{\alpha}^{\prime}\left(\frac{n^{1 / \alpha}\left(\bar{Y}_{1}^{n, \beta, x_{0}}-\varsigma_{1}^{n, \theta, x_{0}}\right)}{\sigma}\right)}{\varphi_{\alpha}\left(\frac{n^{1 / \alpha}\left(\bar{Y}_{1}^{n, \beta, x_{0}}-\varsigma_{1}^{n, \theta, x_{0}}\right)}{\sigma}\right)}\right]+o(1)
$$

where the $o(1)$ term is uniform with respect to $x_{0}$. Now, using Proposition 3.4, we get

$$
\begin{aligned}
d_{1}^{n, \sigma, x_{0}}= & \mathbb{E}\left[\frac{\mathcal{H}_{\bar{Y}_{1}^{n, \beta, x_{0}}}\left(\partial_{\sigma} \bar{Y}_{1}^{n, \beta, x_{0}}\right)}{\sigma}\right] \\
& +\mathbb{E}\left[\frac{\mathcal{H}_{\bar{Y}_{1}^{n, \beta, x_{0}}\left(\partial_{\sigma} \bar{Y}_{1}^{n, \beta, x_{0}}\right)} \frac{n^{1 / \alpha}\left(\bar{Y}_{1}^{n, \beta, x_{0}}-\varsigma_{1}^{n, \theta, x_{0}}\right)}{\sigma} \varphi_{\alpha}^{\prime}\left(\frac{n^{1 / \alpha}\left(\bar{Y}_{1}^{n, \beta, x_{0}}-\varsigma_{1}^{n, \theta, x_{0}}\right)}{\sigma}\right)}{\varphi_{\alpha}\left(\frac{n^{1 / \alpha}\left(\bar{Y}_{1}^{n, \beta, x_{0}}-\varsigma_{1}^{n, \theta, x_{0}}\right)}{\sigma}\right)}\right]+o(1) .
\end{aligned}
$$

From Lemma 5.1 and the convergence result (3.8) we can deduce that

$$
d_{1}^{n, \sigma, x_{0}} \stackrel{n \rightarrow \infty}{\longrightarrow} \frac{1}{\sigma^{2}} \mathbb{E}\left[\left(L_{1}^{\alpha} \mathcal{H}_{L^{\alpha}}(1)-1\right) \frac{\varphi_{\alpha}\left(L_{1}^{\alpha}\right)+L_{1}^{\alpha} \varphi_{\alpha}^{\prime}\left(L_{1}^{\alpha}\right)}{\varphi_{\alpha}\left(L_{1}^{\alpha}\right)}\right]
$$

uniformly with respect to $x_{0}$. Then, the relation (3.10) enables to rewrite this convergence as,

$$
d_{1}^{n, \sigma, x_{0}} \stackrel{n \rightarrow \infty}{\longrightarrow}-\frac{1}{\sigma^{2}} \mathbb{E}\left[\frac{\left(\varphi_{\alpha}\left(L_{1}^{\alpha}\right)+L_{1}^{\alpha} \varphi_{\alpha}^{\prime}\left(L_{1}^{\alpha}\right)\right)^{2}}{\varphi_{\alpha}\left(L_{1}^{\alpha}\right)^{2}}\right] .
$$

This result implies (5.12) and hence (5.10). 
Lemma 5.4. We have the convergence in law,

$$
\left(\begin{array}{c}
-n^{-1 / 2} \sum_{i=0}^{n-1} \frac{\varphi_{\alpha}^{\prime}\left(n^{1 / \alpha}\left(L_{\frac{i+1}{n}}-L_{\frac{i}{n}}\right)\right)}{\varphi_{\alpha}\left(n^{1 / \alpha}\left(L_{\frac{i+1}{n}}-L_{\frac{i}{n}}\right)\right)} \frac{1}{\sigma} \partial_{\theta} b\left(X_{\frac{i}{n}}^{\beta}, \theta\right) \\
-n^{-1 / 2} \sum_{i=0}^{n-1} \frac{1}{\sigma} \frac{\varphi_{\alpha}\left(n^{1 / \alpha}\left(L_{\frac{i+1}{n}}-L_{\frac{i}{n}}\right)\right)+n^{1 / \alpha}\left(L_{\frac{i+1}{n}}-L_{\frac{i}{n}}\right) \varphi_{\alpha}^{\prime}\left(n^{1 / \alpha}\left(L_{\frac{i+1}{n}}-L_{\frac{i}{n}}\right)\right.}{\varphi_{\alpha}\left(n^{1 / \alpha}\left(L_{\frac{i+1}{n}}-L_{\frac{i}{n}}\right)\right)}
\end{array}\right) \Rightarrow \mathcal{N}(0, \mathcal{I}(\beta))
$$

where the convergence is stable with respect to the $\sigma$-field $\sigma\left(L_{s}, s \leq 1\right)$.

Proof. We define the following processes:

$$
\begin{aligned}
& Z_{t}^{n}=\sum_{i=0}^{\lfloor n t\rfloor}\left(L_{\frac{i+1}{n}}-L_{\frac{i}{n}}\right), \\
& \Gamma_{t}^{n}=\left(\begin{array}{c}
\Gamma_{t}^{n, 1} \\
\Gamma_{t}^{n, 2}
\end{array}\right)=n^{-1 / 2}\left(\begin{array}{c}
\sum_{i=0}^{\lfloor n t\rfloor} \frac{\varphi_{\alpha}^{\prime}\left(n^{1 / \alpha}\left(L_{\frac{i+1}{n}}-L_{\frac{i}{n}}\right)\right)}{\varphi_{\alpha}\left(n^{1 / \alpha}\left(L_{\frac{i+1}{n}}-L_{\frac{i}{n}}\right)\right)} \frac{1}{\sigma} \partial_{\theta} b\left(X_{\frac{i}{n}}^{\beta}, \theta\right) \\
\left.\sum_{i=0}^{\lfloor n t\rfloor} \frac{\varphi_{\alpha}\left(n^{1 / \alpha}\left(L_{\frac{i+1}{n}}-L_{\frac{i}{n}}\right)\right)+n^{1 / \alpha}\left(L_{\frac{i+1}{n}}-L_{\frac{i}{n}}\right) \varphi_{\alpha}^{\prime}\left(n^{1 / \alpha}\left(L_{\frac{i+1}{n}}-L_{\frac{i}{n}}\right)\right)}{\frac{1}{\sigma}}\right)
\end{array}\right), \\
& \Gamma_{t}^{\prime n}=\left(\begin{array}{l}
\Gamma_{t}^{\prime n, 1} \\
\Gamma_{t}^{\prime n, 2}
\end{array}\right)=n^{-1 / 2}\left(\begin{array}{c}
\sum_{i=0}^{[n t]} \frac{\varphi_{\alpha}^{\prime}\left(n^{1 / \alpha}\left(L_{\frac{i+1}{n}}-L_{\frac{i}{n}}\right)\right)}{\varphi_{\alpha}\left(n^{1 / \alpha}\left(L_{\frac{i+1}{n}}-L_{\frac{i}{n}}\right)\right)} \\
\sum_{i=0}^{[n t]} \frac{\varphi_{\alpha}\left(n^{1 / \alpha}\left(L_{\frac{i+1}{n}}-L_{\frac{i}{n}}\right)\right)+n^{1 / \alpha}\left(L_{\frac{i+1}{n}}-L_{\frac{i}{n}}\right) \varphi_{\alpha}^{\prime}\left(n^{1 / \alpha}\left(L_{\frac{i+1}{n}}-L_{\frac{i}{n}}\right)\right)}{\varphi_{\alpha}\left(n^{1 / \alpha}\left(L_{\frac{i+1}{n}}-L_{\frac{i}{n}}\right)\right)}
\end{array}\right) .
\end{aligned}
$$

We will apply Lemma 2.8 in [13] to prove (5.13). Indeed, we will show that there exists a Gaussian random vector $\gamma$ with $\operatorname{var}(\gamma)=\left(\begin{array}{cc}\mathbb{E}\left[\frac{\varphi_{\alpha}^{\prime}\left(L_{1}^{\alpha}\right)^{2}}{\varphi_{\alpha}\left(L_{1}^{\alpha}\right)^{2}}\right] & 0 \\ 0 & \mathbb{E}\left[\frac{\left(\varphi_{\alpha}\left(L_{1}^{\alpha}\right)+L_{1}^{\alpha} \varphi_{\alpha}^{\prime}\left(L_{1}^{\alpha}\right)\right)^{2}}{\varphi_{\alpha}\left(L_{1}^{\alpha}\right)^{2}}\right]\end{array}\right)$, independent of $L_{1}$ such that one has the convergence in law

$$
\left(\Gamma_{1}^{\prime n}, Z_{1}^{n}\right) \Rightarrow\left(\gamma, L_{1}\right)
$$

Then, by application of Lemma 2.8 in [13], there exists a bi-dimensional Brownian motion $\left(\Gamma_{t}^{\prime}\right)_{t}$ independent of $\left(L_{t}\right)_{t}$ such that one has the convergence in law $\left(Z^{n}, \Gamma^{n}, \Gamma^{\prime n}\right) \Rightarrow\left(L, \Gamma, \Gamma^{\prime}\right)$ where $\Gamma_{t}=\int_{0}^{t}\left(\begin{array}{cc}\frac{1}{\sigma} \partial_{\theta} b\left(X_{s}^{\beta}, \theta\right) & 0 \\ 0 & \frac{1}{\sigma}\end{array}\right) d \Gamma_{s}^{\prime}$ and $\operatorname{var}\left(\Gamma_{1}^{\prime}\right)=\operatorname{var}(\gamma)$.

Let us focus on the derivation of the convergence (5.14). For $(u, v, w) \in \mathbb{R}^{3}$, we set

$$
\mathcal{X}_{n}(u, v, w)=\mathbb{E}\left[\exp \left(i \frac{u}{n^{1 / 2}} \frac{\varphi_{\alpha}^{\prime}\left(n^{1 / \alpha} L_{\frac{1}{n}}\right)}{\varphi_{\alpha}\left(n^{1 / \alpha} L_{\frac{1}{n}}\right)}+i \frac{v}{n^{1 / 2}} \frac{\left(\varphi_{\alpha}\left(n^{1 / \alpha} L_{\frac{1}{n}}\right)+n^{1 / \alpha} L_{\frac{1}{n}} \varphi_{\alpha}^{\prime}\left(n^{1 / \alpha} L_{\frac{1}{n}}\right)\right)}{\varphi_{\alpha}\left(n^{1 / \alpha} L_{\frac{1}{n}}\right)}+i w L_{\frac{1}{n}}\right)\right]
$$


Using the i.i.d. structure of the increments of the Lévy process $L$, we easily get the following expression about the characteristic function of $\left(\Gamma_{1}^{\prime n}, Z_{1}^{n}\right)$

$$
\log \mathbb{E}\left[\exp \left(i u \Gamma_{1}^{\prime n, 1}+i v \Gamma_{1}^{\prime n, 2}+i w Z_{1}^{n}\right)\right]=n \log \mathcal{X}_{n}(u, v, w)
$$

Let us study the asymptotic behavior of $\mathcal{X}_{n}(u, v, w)$. Using the expansion of the exponential function near zero and that $\frac{\varphi_{\alpha}^{\prime}}{\varphi_{\alpha}}$ and $\frac{x \varphi_{\alpha}^{\prime}(x)}{\varphi_{\alpha}(x)}$ are bounded we get

$$
\begin{aligned}
\mathcal{X}_{n}(u, v, w)= & \mathbb{E}\left\{[ e ^ { i w L _ { n } } ] \left[1+\left(\frac{i u}{n^{1 / 2}} \frac{\varphi_{\alpha}^{\prime}\left(n^{1 / \alpha} L_{\frac{1}{n}}\right)}{\varphi_{\alpha}\left(n^{1 / \alpha} L_{\frac{1}{n}}\right)}+\frac{i v}{n^{1 / 2}} \frac{\left(\varphi_{\alpha}\left(n^{1 / \alpha} L_{\frac{1}{n}}\right)+n^{1 / \alpha} L_{\frac{1}{n}} \varphi_{\alpha}^{\prime}\left(n^{1 / \alpha} L_{\frac{1}{n}}\right)\right)}{\varphi_{\alpha}\left(n^{1 / \alpha} L_{\frac{1}{n}}\right)}\right)\right.\right. \\
& \left.\left.+\frac{1}{2}\left(\frac{i u}{n^{1 / 2}} \frac{\left(\varphi_{\alpha}^{\prime}\left(n^{1 / \alpha} L_{\frac{1}{n}}\right)\right)}{\varphi_{\alpha}\left(n^{1 / \alpha} L_{\frac{1}{n}}\right)}+\frac{i v}{n^{1 / 2}} \frac{\left(\varphi_{\alpha}\left(n^{1 / \alpha} L_{\frac{1}{n}}\right)+n^{1 / \alpha} L_{\frac{1}{n}} \varphi_{\alpha}^{\prime}\left(n^{1 / \alpha} L_{\frac{1}{n}}\right)\right)}{\varphi_{\alpha}\left(n^{1 / \alpha} L_{\frac{1}{n}}\right)}\right)\right)^{2}\right]+O\left(n^{-3 / 2}\right) \\
= & \mathbb{E}\left[e^{i w L_{\frac{1}{n}}}\right]+\frac{i u}{n^{1 / 2}} \mathbb{E}\left[\frac{\varphi_{\alpha}^{\prime}\left(n^{1 / \alpha} L_{\frac{1}{n}}\right)}{\varphi_{\alpha}\left(n^{1 / \alpha} L_{\frac{1}{n}}\right)} e^{i w L_{\frac{1}{n}}}\right]+\frac{i v}{n^{1 / 2}} \mathbb{E}\left[\frac{\left(\varphi_{\alpha}\left(n^{1 / \alpha} L_{\frac{1}{n}}\right)+n^{1 / \alpha} L_{\frac{1}{n}} \varphi_{\alpha}^{\prime}\left(n^{1 / \alpha} L_{\frac{1}{n}}\right)\right)}{\varphi_{\alpha}\left(n^{1 / \alpha} L_{\frac{1}{n}}\right)} e^{i w L_{\frac{1}{n}}}\right] \\
& -\frac{u^{2}}{2 n} \mathbb{E}\left[\left(\frac{\varphi_{\alpha}^{\prime}\left(n^{1 / \alpha} L_{\frac{1}{n}}\right)}{\varphi_{\alpha}\left(n^{1 / \alpha} L_{\frac{1}{n}}\right)}\right)^{2} e^{i w L_{\frac{1}{n}}}\right]-\frac{u v}{n} \mathbb{E}\left[\frac{\varphi_{\alpha}^{\prime}\left(n^{1 / \alpha} L_{\frac{1}{n}}\right)}{\varphi_{\alpha}\left(n^{1 / \alpha} L_{\frac{1}{n}}\right)} \frac{\left(\varphi_{\alpha}\left(n^{1 / \alpha} L_{\frac{1}{n}}\right)+n^{1 / \alpha} L_{\frac{1}{n}} \varphi_{\alpha}^{\prime}\left(n^{1 / \alpha} L_{\frac{1}{n}}\right)\right)}{\varphi_{\alpha}\left(n^{1 / \alpha} L_{\frac{1}{n}}\right)} e^{i w L_{\frac{1}{n}}}\right] \\
& -\frac{v^{2}}{2 n} \mathbb{E}\left[\left(\frac{\varphi_{\alpha}\left(n^{1 / \alpha} L_{\frac{1}{n}}\right)+n^{1 / \alpha} L_{\frac{1}{n}} \varphi_{\alpha}^{\prime}\left(n^{1 / \alpha} L_{\frac{1}{n}}\right)}{\varphi_{\alpha}\left(n^{1 / \alpha} L_{\frac{1}{n}}\right)} e^{i w L_{\frac{1}{n}}}\right]+O\left(n^{-3 / 2}\right)\right. \\
:= & \mathcal{X}_{n}^{(1)}(u, v, w)+\frac{i u}{n^{1 / 2}} \mathcal{X}_{n}^{(2)}(u, v, w)+\frac{i v}{n^{1 / 2}} \mathcal{X}_{n}^{(3)}(u, v, w)-\frac{u^{2}}{2 n} \mathcal{X}_{n}^{(4)}(u, v, w) \\
& -\frac{u v}{n} \mathcal{X}_{n}^{(5)}(u, v, w)-\frac{v^{2}}{2 n} \mathcal{X}_{n}^{(6)}(u, v, w)+O\left(n^{-3 / 2}\right) .
\end{aligned}
$$

First, we have

$$
\mathcal{X}_{n}^{(1)}(u, v, w)=e^{\psi(w) / n}=1+\psi(w) / n+O\left(n^{-2}\right)
$$

where $\psi(w)$ is the Lévy Khintchine exponent of $L_{1}$.

We now focus on the term $\mathcal{X}_{n}^{(2)}(u, v, w)$. Using the results of Lemma 3.3, and the fact that $n^{1 / \alpha} L_{1 / n}$ has the same law as $L_{1}^{n}$, we get

$$
\begin{aligned}
\mathcal{X}_{n}^{(2)}(u, v, w) & =\mathbb{E}\left[\frac{\varphi_{\alpha}^{\prime}}{\varphi_{\alpha}}\left(L_{1}^{\alpha}\right) e^{i \frac{w L_{1}^{\alpha}}{n^{1 / \alpha}}}\right]+O\left(n^{-1}\right) \\
& =\int_{\mathbb{R}} \varphi_{\alpha}^{\prime}(s) e^{i \frac{w s}{n^{1 / \alpha}}} d s+O\left(n^{-1}\right) \\
& =-\frac{i w}{n^{1 / \alpha}} \int_{\mathbb{R}} \varphi_{\alpha}(s) e^{i \frac{w s}{n^{1 / \alpha}}} d s+O\left(n^{-1}\right) \quad \text { using integration by parts formula } \\
& =O\left(\max \left(n^{-1 / \alpha}, n^{-1}\right)\right) .
\end{aligned}
$$


For the term $\mathcal{X}_{n}^{(3)}(u, v, w)$, using Lemma 3.3 again, it is easy to see that

$$
\begin{aligned}
\mathcal{X}_{n}^{(3)}(u, v, w) & =\mathbb{E}\left[\left(\frac{\varphi_{\alpha}\left(L_{1}^{\alpha}\right)+L_{1}^{\alpha} \varphi_{\alpha}^{\prime}\left(L_{1}^{\alpha}\right)}{\varphi_{\alpha}\left(L_{1}^{\alpha}\right)}\right) e^{\left.i \frac{w L_{1}^{\alpha}}{n^{1 / \alpha}}\right]}\right]+O\left(n^{-1}\right) \\
& =\int_{\mathbb{R}} \varphi_{\alpha}(s) e^{i \frac{w s}{n^{1 / \alpha}}} \mathrm{d} s+\int_{\mathbb{R}} s \varphi_{\alpha}^{\prime}(s) e^{i \frac{w s}{n^{1 / \alpha}}} \mathrm{d} s+O\left(n^{-1}\right) .
\end{aligned}
$$

Using integration by parts formula, we have

$$
\mathbb{E}\left(e^{i w L_{\frac{1}{n}}^{\alpha}}\right)=\int_{\mathbb{R}} \varphi_{\alpha}(s) e^{\frac{i w s}{n^{1 / \alpha}}} \mathrm{d} s=-\frac{n^{1 / \alpha}}{i w} \int_{\mathbb{R}} \varphi_{\alpha}^{\prime}(s) e^{\frac{i w s}{n^{1 / \alpha}}} \mathrm{d} s .
$$

Then, we deduce that

$$
\int_{\mathbb{R}} \varphi_{\alpha}^{\prime}(s) e^{\frac{i w s}{n^{1 / \alpha}}} \mathrm{d} s=-\frac{i w}{n^{1 / \alpha}} \mathbb{E}\left(e^{i w L_{\frac{1}{n}}^{\alpha}}\right)=-\frac{i w}{n^{1 / \alpha}} \mathbb{E}\left(e^{\frac{i w}{n^{1 / \alpha}} L_{1}^{\alpha}}\right) .
$$

Since $L_{1}^{\alpha}$ is a symmetric $\alpha$-stable process then we have for some constant $C(\alpha)>0$

$$
\mathbb{E}\left(e^{\frac{i w}{n^{1 / \alpha}} L_{1}^{\alpha}}\right)=e^{-C(\alpha)\left|\frac{w}{n^{1 / \alpha}}\right|^{\alpha}} .
$$

Combining (5.20) with (5.21), we have

$$
\int_{\mathbb{R}} \varphi_{\alpha}^{\prime}(s) e^{\frac{i w s}{n^{1 / \alpha}}} \mathrm{d} s=-\frac{i w}{n^{1 / \alpha}} e^{-C(\alpha)\left|\frac{w}{n^{1 / \alpha}}\right|^{\alpha}}
$$

Now, since $\int\left|s \varphi_{\alpha}^{\prime}(s)\right| \mathrm{d} s<\infty$ and $w \mapsto w e^{-C(\alpha)\left|\frac{w}{n^{1 / \alpha}}\right|^{\alpha}}$ admits a derivative on $\mathbb{R}$, we obtain by taking the derivative with respect to $w$ of the both sides of (5.22)

$$
\begin{aligned}
\int_{\mathbb{R}} s \varphi_{\alpha}^{\prime}(s) e^{\frac{i w s}{n^{1 / \alpha}}} \mathrm{d} s & =-e^{-C(\alpha)\left|\frac{w}{n^{1 / \alpha}}\right|^{\alpha}}+\alpha C(\alpha) e^{-C(\alpha)\left|\frac{w}{n^{1 / \alpha}}\right|^{\alpha}} \frac{|w|^{\alpha}}{n} \\
& =-\int_{\mathbb{R}} \varphi_{\alpha}(s) e^{\frac{i w s}{n^{1 / \alpha}}} \mathrm{d} s+\alpha C(\alpha) e^{-C(\alpha)\left|\frac{w}{n^{1 / \alpha}}\right|^{\alpha}} \frac{|w|^{\alpha}}{n} .
\end{aligned}
$$

From (5.19) and (5.23) we can deduce that

$$
\mathcal{X}_{n}^{(3)}(u, v, w)=O\left(n^{-1}\right)
$$

For the term $\mathcal{X}_{n}^{(4)}(u, v, w)$, using Lemma 3.3 again, we have

$$
\mathcal{X}_{n}^{(4)}(u, v, w)=\mathbb{E}\left[\frac{\varphi_{\alpha}^{\prime}}{\varphi_{\alpha}}\left(L_{1}^{\alpha}\right)^{2} e^{i \frac{w L_{1}^{\alpha}}{n^{1 / \alpha}}}\right]+O\left(n^{-1}\right) \stackrel{n \rightarrow \infty}{\longrightarrow} \mathbb{E}\left[\frac{\varphi_{\alpha}^{\prime}}{\varphi_{\alpha}}\left(L_{1}^{\alpha}\right)^{2}\right]
$$

For the term $\mathcal{X}_{n}^{(5)}(u, v, w)$ we have

$$
\mathcal{X}_{n}^{(5)}(u, v, w)=\mathbb{E}\left[\frac{\varphi_{\alpha}^{\prime}\left(L_{1}^{\alpha}\right)}{\varphi_{\alpha}\left(L_{1}^{\alpha}\right)} \frac{\left(\varphi_{\alpha}\left(L_{1}^{\alpha}\right)+L_{1}^{\alpha} \varphi_{\alpha}^{\prime}\left(L_{1}^{\alpha}\right)\right)}{\varphi_{\alpha}\left(L_{1}^{\alpha}\right)} e^{\frac{i w L_{1}^{\alpha}}{n^{1 / \alpha}}}\right]+O\left(n^{-1}\right)
$$




$$
\stackrel{n \rightarrow \infty}{\longrightarrow} \mathbb{E}\left[\frac{\varphi_{\alpha}^{\prime}\left(L_{1}^{\alpha}\right)}{\varphi_{\alpha}\left(L_{1}^{\alpha}\right)} \frac{\left(\varphi_{\alpha}\left(L_{1}^{\alpha}\right)+L_{1}^{\alpha} \varphi_{\alpha}^{\prime}\left(L_{1}^{\alpha}\right)\right)}{\varphi_{\alpha}\left(L_{1}^{\alpha}\right)}\right]=0 \quad \text { from }(3.13)
$$

For the term $\mathcal{X}_{n}^{(6)}(u, v, w)$, we see that

$$
\begin{aligned}
& \mathcal{X}_{n}^{(6)}(u, v, w)=\mathbb{E}\left[\left(\frac{\varphi_{\alpha}^{\prime}\left(L_{1}^{\alpha}\right)+L_{1}^{\alpha} \varphi_{\alpha}^{\prime}\left(L_{1}^{\alpha}\right)}{\varphi_{\alpha}\left(L_{1}^{\alpha}\right)}\right)^{2} e^{\frac{i w L_{1}^{\alpha}}{n^{1 / \alpha}}}\right]+O\left(n^{-1}\right) \\
& \stackrel{n \rightarrow \infty}{\longrightarrow} \mathbb{E}\left[\left(\frac{\varphi_{\alpha}^{\prime}\left(L_{1}^{\alpha}\right)+L_{1}^{\alpha} \varphi_{\alpha}^{\prime}\left(L_{1}^{\alpha}\right)}{\varphi_{\alpha}\left(L_{1}^{\alpha}\right)}\right)^{2}\right] .
\end{aligned}
$$

Collecting the convergence of $\left(\mathcal{X}_{n}^{(i)}(u, v, w)\right)_{1 \leq i \leq 6}$, we deduce the convergence

$$
\log \mathbb{E}\left[\exp \left(i u \Gamma_{1}^{\prime n, 1}+i v \Gamma_{1}^{\prime n, 2}+i w Z_{1}^{n}\right)\right] \stackrel{n \rightarrow \infty}{\longrightarrow} \psi(v)-\frac{u^{2}}{2} \mathbb{E}\left[\frac{\varphi_{\alpha}^{\prime}}{\varphi_{\alpha}}\left(L_{1}^{\alpha}\right)^{2}\right]-\frac{v^{2}}{2} \mathbb{E}\left[\left(\frac{\varphi_{\alpha}^{\prime}\left(L_{1}^{\alpha}\right)+L_{1}^{\alpha} \varphi_{\alpha}^{\prime}\left(L_{1}^{\alpha}\right)}{\varphi_{\alpha}\left(L_{1}^{\alpha}\right)}\right)^{2}\right]
$$

and thus the convergence in law of this lemma.

\section{Proofs of Lemmas 3.5-3.8}

The proof of these lemmas is very technical and requires many intermediate results. We first recall the Malliavin calculus for jump processes used in [6] and some properties of the Malliavin weights. Next we will establish a regularity property of a conditional expectation with respect to the conditioning variable. Then we will proceed to the proof of the lemmas.

\subsection{Malliavin calculus and preliminary lemmas}

We recall the Malliavin calculus on the Poisson space associated to the measure $\mu^{(n)}$ (defined in Sect. 3.2.1) and the basic properties of the Malliavin operators (see Bichteler, Gravereaux, Jacod [4], Chap. IV, Sects. 8-10). For a test function $f:[0,1] \times E \mapsto \mathbb{R}\left(f\right.$ is measurable, $\mathcal{C}^{2}$ with respect to the second variable, with bounded derivative, and $\left.f \in \cap_{p \geq 1} \mathbf{L}^{p}(\nu)\right)$ we set $\mu^{(n)}(f)=\int_{0}^{1} \int_{E} f(t, z) \mu^{(n)}(\mathrm{d} t, \mathrm{~d} z)$. We introduce an auxiliary function $\rho^{n}$ as

$$
\rho^{n}(z)= \begin{cases}z^{4} & \text { if }|z|<1 \\ \zeta(z) & \text { if } 1 \leq|z| \leq 2 \\ z^{2} \tau\left(\frac{z}{2 n^{1 / \alpha}}\right) & \text { if } \quad|z|>2\end{cases}
$$

where $\tau$ is defined in the assumption $\mathbf{H}_{1}\left(b_{i}\right)$, and $\zeta$ is a non negative function belonging to $\mathbf{C}^{\infty}$ such that the function $\rho^{n}$ belongs to $\mathbf{C}^{\infty}$. Note that $\zeta$ is defined such that $\rho^{n}(z)$ admits a derivative and $\rho^{n},\left(\rho^{n}\right)^{\prime}, \rho^{n} \frac{F_{n}^{\prime}(z)}{F_{n}(z)}$ belong to

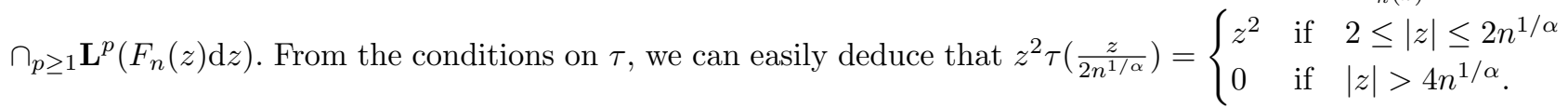

Moreover, we can see that $\rho^{n}(z) \stackrel{n \rightarrow \infty}{\longrightarrow} \rho(z)$ where

$$
\rho(z)=\left\{\begin{array}{lll}
z^{4} & \text { if } \quad|z|<1 \\
\zeta(z) & \text { if } \quad 1 \leq|z| \leq 2 \\
z^{2} & \text { if } \quad|z|>2
\end{array}\right.
$$


Note that from the definition of $\rho^{n}$ and $\rho$, we can easily see that $\rho^{n}(z)=\rho(z)$ if $|z| \leq 2 n^{1 / \alpha}$.

With these notations, we define the Malliavin operator $L$, on a simple functional $\mu^{(n)}(f)$, in the same way as in [5] by the following equations :

$$
L\left(\mu^{(n)}(f)\right)=\frac{1}{2} \mu^{(n)}\left(\left(\rho^{n}\right)^{\prime} f^{\prime}+\rho^{n} \frac{F_{n}^{\prime}}{F_{n}} f^{\prime}+\rho^{n} f^{\prime \prime}\right)
$$

where $f^{\prime}$ and $f^{\prime \prime}$ are the derivatives with respect to the second variable. For $\Phi=F\left(\mu^{(n)}\left(f_{1}\right), . ., \mu^{(n)}\left(f_{k}\right)\right)$, with $F$ of $\operatorname{class} \mathcal{C}^{2}$, we set

$$
L \Phi=\sum_{i=1}^{k} \frac{\partial F}{\partial x_{i}}\left(\mu^{(n)}\left(f_{1}\right), \ldots, \mu^{(n)}\left(f_{k}\right)\right) L\left(\mu^{(n)}\left(f_{i}\right)\right)+\frac{1}{2} \sum_{i, j=1}^{k} \frac{\partial^{2} F}{\partial x_{i} \partial x_{j}}\left(\mu^{(n)}\left(f_{1}\right), \ldots, \mu^{(n)}\left(f_{k}\right)\right) \mu^{(n)}\left(\rho^{n} f_{i}^{\prime} f_{j}^{\prime}\right)
$$

These definitions permit to construct a linear operator $L$ on a space $D \subset \cap_{p \geq 1} \mathbf{L}^{p}$ whose basic properties are the following.

i) $L$ is self-adjoint: $\forall \Phi, \Psi \in D$, we have $\mathbb{E} \Phi L \Psi=\mathbb{E} L \Phi \Psi$.

ii) $L \Phi^{2} \geq 2 \Phi L \Phi$.

iii) $\mathbb{E} L \Phi=0$.

We associate to $L$, the symmetric bilinear operator $\Gamma$ :

$$
\Gamma(\Phi, \Psi)=L(\Phi \Psi)-\Phi L \Psi-\Psi L \Phi
$$

This operator satisfies the following properties (see [4], Eqs. (8-3))

$$
\begin{aligned}
\Gamma(F(\Phi), \Psi) & =F^{\prime}(\Phi) \Gamma(\Phi, \Psi), \\
\Gamma\left(F\left(\Phi_{1}, \Phi_{2}\right), \Psi\right) & =\partial_{\Phi_{1}} F\left(\Phi_{1}, \Phi_{2}\right) \Gamma\left(\Phi_{1}, \Psi\right)+\partial_{\Phi_{2}} F\left(\Phi_{1}, \Phi_{2}\right) \Gamma\left(\Phi_{2}, \Psi\right), \\
|\Gamma(\Phi, \Psi)| & \leq \Gamma(\Phi, \Phi)^{1 / 2} \Gamma(\Psi, \Psi)^{1 / 2} .
\end{aligned}
$$

Remark 6.1. The operators $L$ and $\Gamma$ depend on $n$ through the functions $\rho^{n}$ and $F_{n}$ but to simplify the notation we omit the dependence in $n$.

The operator $L$ and the operator $\Gamma$ permit to establish the following integration by parts formula (see [4], Prop. 8-10, p. 103).

Proposition 6.2. For $\Phi$ and $\Psi$ in $D$, and $f$ bounded with bounded derivative up to order two, if $\Gamma(\Phi, \Phi)$ is invertible and $\Gamma^{-1}(\Phi, \Phi) \in \cap_{p \geq 1} \boldsymbol{L}^{p}$ then we have

$$
\mathbb{E} f^{\prime}(\Phi) \Psi=\mathbb{E} f(\Phi) \mathcal{H}_{\Phi}(\Psi),
$$

with

$$
\begin{aligned}
\mathcal{H}_{\Phi}(\Psi) & =-2 \Psi \Gamma^{-1}(\Phi, \Phi) L \Phi-\Gamma\left(\Phi, \Psi \Gamma^{-1}(\Phi, \Phi)\right) \\
& =-2 \Psi \Gamma^{-1}(\Phi, \Phi) L \Phi-\frac{1}{\Gamma(\Phi, \Phi)} \Gamma(\Phi, \Psi)+\frac{\Psi}{\Gamma(\Phi, \Phi)^{2}} \Gamma(\Phi, \Gamma(\Phi, \Phi)) \\
& =\Phi L\left(\frac{\Psi}{\Gamma(\Phi, \Phi)}\right)-\frac{\Psi L \Phi}{\Gamma(\Phi, \Phi)}-L\left(\frac{\Psi \Phi}{\Gamma(\Phi, \Phi)}\right) .
\end{aligned}
$$


With these notations, we can explicit the Malliavin weight $\mathcal{H}_{\bar{Y}_{1}^{n, \beta, x_{0}}}\left(\nabla_{\beta} \bar{Y}_{1}^{n, \beta, x_{0}}\right)$ appearing in the representation of the score function given in Proposition 3.4.

Proposition 6.3 (Thms. 3.1 and 6.2 in $[6]$ ).

$$
\begin{aligned}
\mathcal{H}_{\bar{Y}_{1}^{n, \beta, x_{0}}}\left(\nabla_{\beta} \bar{Y}_{1}^{n, \beta, x_{0}}\right) & =\left(\begin{array}{l}
\mathcal{H}_{\bar{Y}_{1}^{n, \beta, x_{0}}}\left(\partial_{\theta} \bar{Y}_{1}^{n, \beta, x_{0}}\right) \\
\mathcal{H}_{\bar{Y}_{1}^{n, \beta, x_{0}}}\left(\partial_{\sigma} \bar{Y}_{1}^{n, \beta, x_{0}}\right)
\end{array}\right) \\
& =\left(\begin{array}{c}
\partial_{\theta} \bar{Y}_{1}^{n, \beta, x_{0}} \\
\partial_{\sigma} \bar{Y}_{1}^{n, \beta, x_{0}}
\end{array}\right) \mathcal{H}_{\bar{Y}_{1}^{n, \beta, x_{0}}}(1)-\frac{1}{\Gamma\left(\bar{Y}_{1}^{n, \beta, x_{0}}, \bar{Y}_{1}^{n, \beta, x_{0}}\right)}\left(\begin{array}{c}
\Gamma\left(\bar{Y}_{1}^{n, \beta, x_{0}}, \partial_{\theta} \bar{Y}_{1}^{n, \beta, x_{0}}\right) \\
\Gamma\left(\bar{Y}_{1}^{n, \beta, x_{0}}, \partial_{\sigma} \bar{Y}_{1}^{n, \beta, x_{0}}\right)
\end{array}\right)
\end{aligned}
$$

and

$$
\begin{aligned}
\mathcal{H}_{\bar{Y}_{1}^{n, \beta, x_{0}}}(1) & =\frac{\Gamma\left(\bar{Y}_{1}^{n, \beta, x_{0}}, \Gamma\left(\bar{Y}_{1}^{n, \beta, x_{0}}, \bar{Y}_{1}^{n, \beta, x_{0}}\right)\right)}{\left(\Gamma\left(\bar{Y}_{1}^{n, \beta, x_{0}}, \bar{Y}_{1}^{n, \beta, x_{0}}\right)\right)^{2}}-2 \frac{L \bar{Y}_{1}^{n, \beta, x_{0}}}{\Gamma\left(\bar{Y}_{1}^{n, \beta, x_{0}}, \bar{Y}_{1}^{n, \beta, x_{0}}\right)} \\
& =\frac{1}{\sigma} n^{1 / \alpha} \widehat{\mathcal{H}}_{\beta}^{n}(1)+\mathcal{R}_{1, \beta}^{n}(1)+\mathcal{R}_{2, \beta}^{n}(1)+\mathcal{R}_{3, \beta}^{n}(1) .
\end{aligned}
$$

The main term $\widehat{\mathcal{H}}_{\beta}^{n}(1)$ is given by

$$
\widehat{\mathcal{H}}_{\beta}^{n}(1)=\frac{\int_{0}^{1} \int_{\mathbb{R}}\left(\epsilon_{s}^{n}\right)^{-3} \rho^{n}(z)\left(\rho^{n}\right)^{\prime}(z) \mu^{n}(\mathrm{~d} s, \mathrm{~d} z)}{\epsilon_{1}^{n}\left[\int_{0}^{1} \int_{\mathbb{R}}\left(\epsilon_{s}^{n}\right)^{-2} \rho^{n}(z) \mu^{n}(\mathrm{~d} s, \mathrm{~d} z)\right]^{2}}-\frac{\int_{0}^{1} \int_{\mathbb{R}}\left(\epsilon_{s}^{n}\right)^{-1}\left[\left(\rho^{n}\right)^{\prime}(z)-\frac{1+\alpha}{z} \rho^{n}(z)\right] \mu^{n}(\mathrm{~d} s, \mathrm{~d} z)}{\epsilon_{1}^{n} \int_{0}^{1} \int_{\mathbb{R}}\left(\epsilon_{s}^{n}\right)^{-2} \rho^{n}(z) \mu^{n}(\mathrm{~d} s, \mathrm{~d} z)}
$$

with

$$
\epsilon_{s}^{n}=\exp \left(\frac{1}{n} \int_{0}^{s} b^{\prime}\left(\bar{Y}_{u}^{n, \beta, x_{0}}, \theta\right) \mathrm{d} u\right)
$$

The remainder terms satisfy for any compact set $Q \subset \mathbb{R} \times(0, \infty)$

$$
\forall p \geq 2, \quad \mathbb{E} \sup _{\beta \in Q}\left|\mathcal{R}_{1, \beta}^{n}(1)\right|^{p} \leq \frac{C}{n}, \quad \sup _{\beta \in Q}\left|\mathcal{R}_{2, \beta}^{n}(1)\right| \leq \frac{C}{n}, \quad \sup _{\beta \in Q}\left|\mathcal{R}_{3, \beta}^{n}(1)\right| \leq \frac{C}{n},
$$

where $C$ is some deterministic constant.

\section{Remark 6.4.}

i) It is proved in [6] (see (4.23)) that $\widehat{\mathcal{H}}_{\beta}^{n}(1)$ is bounded by a random variable independent of $n, \beta$ and $x_{0}$ and belonging to $\cap_{p \geq 1} \mathbf{L}^{p}$ and that it converges in $\mathbf{L}^{p}, \forall p \geq 1$, uniformly with respect to $x_{0}$ (see (4.23) and (5.49) in [6]) to $\mathcal{H}_{L^{\alpha}}(1)$ given by

$$
\mathcal{H}_{L^{\alpha}}(1)=\frac{\int_{0}^{1} \int_{\mathbb{R}} \rho(z) \rho^{\prime}(z) \mu(\mathrm{d} s, \mathrm{~d} z)}{\left[\int_{0}^{1} \int_{\mathbb{R}} \rho(z) \mu(\mathrm{d} s, \mathrm{~d} z)\right]^{2}}-\frac{\int_{0}^{1} \int_{\mathbb{R}}\left[\rho^{\prime}(z)-\frac{1+\alpha}{z} \rho(z)\right] \mu(\mathrm{d} s, \mathrm{~d} z)}{\int_{0}^{1} \int_{\mathbb{R}} \rho(z) \mu(\mathrm{d} s, \mathrm{~d} z)} .
$$

Moreover, $\mathcal{H}_{L^{\alpha}}(1)$ and $L_{1}^{\alpha} \mathcal{H}_{L^{\alpha}}(1)$ belong to $\mathbf{L}^{p}, \forall p \geq 1$. 
ii) In the case $b \equiv 0$ and $\sigma=1$, we have $\epsilon_{s}^{n}=1$ and the remainder terms $\mathcal{R}_{2, \beta}^{n}(1), \mathcal{R}_{3, \beta}^{n}(1)$ are equal to zero (see (4.7) and (4.8) in [6]). Moreover, we see that (6.12) can be rewritten as $\widehat{\mathcal{H}}_{\beta}^{n}(1)+\frac{1}{n^{1 / \alpha}} \mathcal{R}_{1, \beta}^{n}(1)=\mathcal{H}_{L_{1}^{n}}(1)$, then we can deduce that $\mathcal{H}_{L_{1}^{n}}(1) \underset{p \geq 1}{\stackrel{\mathbf{L}^{p}}{\longrightarrow}} \mathcal{H}_{L^{\alpha}}(1)$.

Before studying the Malliavin weight $\mathcal{H}_{\bar{Y}_{1}^{n, \beta, x_{0}}}\left(\nabla_{\beta} \bar{Y}_{1}^{n, \beta, x_{0}}\right)$, we give some control on the processes $\left(\partial_{\theta} Y_{t}^{\beta}\right)_{t}$ and $\left(\partial_{\sigma} Y_{t}^{\beta}\right)_{t}$, respectively solution to the equations

$$
\begin{aligned}
& \partial_{\theta} \bar{Y}_{t}^{n, \beta, x_{0}}=\frac{1}{n} \int_{0}^{t} b^{\prime}\left(\bar{Y}_{s}^{n, \beta, x_{0}}, \theta\right) \partial_{\theta} \bar{Y}_{s}^{n, \beta, x_{0}} \mathrm{~d} s+\frac{1}{n} \int_{0}^{t} \partial_{\theta} b\left(\bar{Y}_{s}^{n, \beta, x_{0}}, \theta\right) \mathrm{d} s, \\
& \partial_{\sigma} \bar{Y}_{t}^{n, \beta, x_{0}}=\frac{1}{n} \int_{0}^{t} b^{\prime}\left(\bar{Y}_{s}^{n, \beta, x_{0}}, \theta\right) \partial_{\sigma} \bar{Y}_{s}^{n, \beta, x_{0}} \mathrm{~d} s+\frac{L_{t}^{n}}{n^{1 / \alpha}} .
\end{aligned}
$$

We have the following properties.

Lemma 6.5 (Lem. 5.1 in [6]). Let $Q \subset \mathbb{R} \times(0, \infty)$ be a compact subset. We have
i) $\sup _{\beta \in Q}\left|\partial_{\theta} \bar{Y}_{1}^{n, \beta, x_{0}}\right| \leq \frac{C}{n}$,
ii) $\sup _{\beta \in Q} \sup _{s \in[0,1]}\left|\partial_{\sigma} \bar{Y}_{s}^{n, \beta, x_{0}}\right| \underset{L^{p}}{\stackrel{n \rightarrow \infty}{\longrightarrow}} 0, \quad \forall p \geq 1$.

We now proceed to the decomposition of the Malliavin weight $\mathcal{H}_{\bar{Y}_{1}^{n, \beta_{n}, x_{0}}}\left(\nabla_{\beta} \bar{Y}_{1}^{n, \beta_{n}, x_{0}}\right)$ defined in Proposition 6.3 into some main parts and some remainder parts. From (6.11), (6.12), we can rewrite $\mathcal{H}_{\bar{Y}_{1}^{n, \beta_{n}, x_{0}}}\left(\nabla_{\beta} \bar{Y}_{1}^{n, \beta_{n}, x_{0}}\right)$ as,

$$
\mathcal{H}_{\bar{Y}_{1}^{n, \beta_{n}, x_{0}}}\left(\nabla_{\beta} \bar{Y}_{1}^{n, \beta_{n}, x_{0}}\right)=\left(\begin{array}{c}
\frac{1}{\sigma_{n}} n^{1 / \alpha} \partial_{\theta} \bar{Y}_{1}^{n, \beta_{n}, x_{0}} \widehat{\mathcal{H}}_{\beta_{n}}^{n}(1) \\
\frac{1}{\sigma_{n}}\left(n^{1 / \alpha} \partial_{\sigma} \bar{Y}_{1}^{n, \beta_{n}, x_{0}} \widehat{\mathcal{H}}_{\beta_{n}}^{n}(1)-1\right)
\end{array}\right)+\mathcal{R}_{\beta_{n}}^{n}\left(\nabla_{\beta} \bar{Y}_{1}^{n, \beta_{n}, x_{0}}\right)
$$

where $\widehat{\mathcal{H}}_{\beta}^{n}(1)$ is given by $(6.13)$ and

$$
\mathcal{R}_{\beta_{n}}^{n}\left(\nabla_{\beta} \bar{Y}_{1}^{n, \beta_{n}, x_{0}}\right)=\left(\begin{array}{c}
\partial_{\theta} \bar{Y}_{1}^{n, \beta_{n}, x_{0}} \\
\partial_{\sigma} \bar{Y}_{1}^{n, \beta_{n}, x_{0}}
\end{array}\right)\left[\mathcal{R}_{1, \beta_{n}}^{n}(1)+\mathcal{R}_{2, \beta_{n}}^{n}(1)+\mathcal{R}_{3, \beta_{n}}^{n}(1)\right]-\left(\begin{array}{c}
\frac{V_{1}^{n, \theta_{n}}}{U_{1}^{n, \beta_{n}}} \\
\frac{V_{1}^{n, \sigma_{n}}}{U_{1}^{n, \beta_{n}}}-\frac{1}{\sigma_{n}}
\end{array}\right)
$$

with $U_{1}^{n, \beta}=\Gamma\left(\bar{Y}_{1}^{n, \beta, x_{0}}, \bar{Y}_{1}^{n, \beta, x_{0}}\right), V_{1}^{n, \theta}=\Gamma\left(\bar{Y}_{1}^{n, \beta, x_{0}}, \partial_{\theta} \bar{Y}_{1}^{n, \beta, x_{0}}\right)$ and $V_{1}^{n, \sigma}=\Gamma\left(\bar{Y}_{1}^{n, \beta, x_{0}}, \partial_{\sigma} \bar{Y}_{1}^{n, \beta, x_{0}}\right)$ given by

$$
\begin{aligned}
U_{1}^{n, \beta}=\frac{\left(\epsilon_{1}^{n}\right)^{2} \sigma^{2}}{n^{2 / \alpha}} \int_{0}^{1} \int_{\mathbb{R}}\left(\epsilon_{s}^{n}\right)^{-2} \rho^{n}(z) \mu^{(n)}(\mathrm{d} s, \mathrm{~d} z), \\
V_{1}^{n, \theta}=\frac{1}{n}\left(\epsilon_{1}^{n}\right)^{2} \int_{0}^{1}\left(\epsilon_{s}^{n}\right)^{-2}\left(U_{s}^{n, \beta}\left[\left(\partial_{\theta} b\right)^{\prime}\left(\bar{Y}_{s}^{n, \beta, x_{0}}, \theta\right)+b^{\prime \prime}\left(\bar{Y}_{s}^{n, \beta, x_{0}}, \theta\right) \partial_{\theta} \bar{Y}_{s}^{n, \beta, x_{0}}\right]\right) \mathrm{d} s, \\
V_{1}^{n, \sigma}=\frac{1}{n}\left(\epsilon_{1}^{n}\right)^{2} \int_{0}^{1}\left(\epsilon_{s}^{n}\right)^{-2}\left(b^{\prime \prime}\left(\bar{Y}_{s}^{n, \beta, x_{0}}, \theta\right) \partial_{\sigma} \bar{Y}_{s}^{n, \beta, x_{0}} U_{s}^{n, \beta}\right) \mathrm{d} s+\frac{\sigma}{n^{2 / \alpha}}\left(\epsilon_{1}^{n}\right)^{2} \int_{0}^{t} \int_{\mathbb{R}}\left(\epsilon_{s}^{n}\right)^{-2} \rho^{n}(z) \mu^{(n)}(\mathrm{d} s, \mathrm{~d} z)
\end{aligned}
$$

and $\left(\epsilon_{s}^{n}\right)_{s \in[0,1]}$ given by $(6.14)$. 
Now we recall two technical lemmas given in [6] useful to study the convergence of the Malliavin weight $\mathcal{H}_{\bar{Y}_{1}^{n, \beta_{n}, x_{0}}}\left(\nabla_{\beta} \bar{Y}_{1}^{n, \beta_{n}, x_{0}}\right)$ in the proof of Lemmas 3.7-3.8 later.

Lemma 6.6 (Lem. 5.4 in [6]). Let $\left(\beta_{n}\right)$ be a sequence converging to $\beta$. For all $p \geq 1$, the following convergences hold uniformly with respect to $x_{0}$

i) $n \partial_{\theta} \bar{Y}_{1}^{n, \beta_{n}, x_{0}} \widehat{\mathcal{H}}_{\beta_{n}}^{n}(1) \stackrel{n \rightarrow \infty}{\underset{L^{p}}{\longrightarrow}} \partial_{\theta} b\left(x_{0}, \theta\right) \mathcal{H}_{L^{\alpha}}(1)$,

ii) $n^{1 / \alpha} \partial_{\sigma} \bar{Y}_{1}^{n, \beta_{n}, x_{0}} \widehat{\mathcal{H}}_{\beta_{n}}^{n}(1) \stackrel{n \rightarrow \infty}{L^{p}} L_{1}^{\alpha} \mathcal{H}_{L^{\alpha}}(1)$,

where $\widehat{\mathcal{H}}_{\beta}^{n}(1)$ and $\mathcal{H}_{L^{\alpha}}(1)$ are respectively given by (6.13) and (6.16).

Lemma 6.7 (Lem. 5.3 in [6]). Let $Q \subset \mathbb{R} \times(0, \infty)$ be a compact subset. The following estimates hold:
i) $\sup _{\beta \in Q}\left|\frac{V_{1}^{n, \theta}}{U_{1}^{n, \beta}}\right| \leq \frac{C}{n}$,
ii) $\frac{V_{1}^{n, \sigma}}{U_{1}^{n, \beta}}=\frac{1}{\sigma}+\mathcal{R}_{9, \beta}^{n}(1)$,

where $C$ is some deterministic constant and $\sup _{\beta \in Q}\left|\mathcal{R}_{9, \beta}^{n}(1)\right|$ converges to zero as $n \rightarrow \infty$ in $\boldsymbol{L}^{p}, \forall p \geq 1$.

\subsection{Regularity of the conditional expectation}

In this section, we prove a regularity property of the conditional expectation with respect to the conditioning variable.

Proposition 6.8. Let $\mathcal{H}$ be a random variable such that $\mathbb{E}(\mathcal{H})^{2}<\infty$. We assume that there exists a sequence of random variables $\left(\mathcal{H}^{n}\right)_{n \geq 1}$ with $\mathbb{E}\left(\mathcal{H}^{n}\right)^{2}<\infty$ and such that $\mathcal{H}^{n} \underset{L^{2}}{\stackrel{n}{\longrightarrow}} \mathcal{H}$ and $\sup _{n}\left\|\Gamma\left(\mathcal{H}^{n}, \mathcal{H}^{n}\right)\right\|_{2}<\infty$. Then,

$$
\mathbb{E}\left[\mathbb{E}\left[\mathcal{H} \mid \bar{Y}_{1}^{n, \beta_{n}, x_{0}}\right]^{2}\right]-\mathbb{E}\left[\mathbb{E}\left[\mathcal{H} \mid L_{1}^{\alpha}\right]^{2}\right] \stackrel{n \rightarrow \infty}{\longrightarrow} 0
$$

and this convergence is uniform with respect to $x_{0}$.

Remark 6.9. Note that if the random variable $\mathcal{H}$ depends on all the measure $\mu$ then the Malliavin calculus of Section 3.2.1 is not defined. So we need to introduce the sequence of random variables $\left(\mathcal{H}^{n}\right)$, for which the Malliavin calculus of Section 6.1 is defined, such that $\Gamma\left(\mathcal{H}^{n}, \mathcal{H}^{n}\right)$ is also well defined. It is the case, for instance, if $\mathcal{H}^{n}$ is a simple functional of $\mu^{(n)}$.

Proof of Proposition 6.8. First we reduce the situation to the case where the random variable in the expectation is bounded. Let $K>1$ and denote by $x \mapsto \mathcal{X}_{K}(x)$ a smooth truncation function with

$$
\left\{\begin{array}{lll}
\mathcal{X}_{K}(x)=0 & \text { for } & |x|>K \\
\mathcal{X}_{K}(x)=1 & \text { for } & |x| \leq K / 2 \\
0 \leq \mathcal{X}_{K}(x) \leq 1 & \text { for } & K / 2 \leq|x| \leq K
\end{array}\right.
$$

For all $\epsilon>0$, we can choose $K$ large enough such that $\left\|\mathcal{H}-\mathcal{H} \mathcal{X}_{K}(\mathcal{H})\right\|_{2}^{2}<\epsilon$ and then, one can see that (6.24) is implied by the following convergence, $\forall K>2$

$$
\sup _{x_{0}}\left|\mathbb{E}\left[\mathbb{E}\left[\mathcal{H} \mathcal{X}_{K}(\mathcal{H}) \mid \bar{Y}_{1}^{n, \beta_{n}, x_{0}}\right]^{2}\right]-\mathbb{E}\left[\mathbb{E}\left[\mathcal{H} \mathcal{X}_{K}(\mathcal{H}) \mid L_{1}^{\alpha}\right]^{2}\right]\right| \stackrel{n \rightarrow \infty}{\longrightarrow} 0 .
$$


Now since $\mathbb{E}\left(\mathcal{H}^{n}-\mathcal{H}\right)^{2} \stackrel{n \rightarrow \infty}{\longrightarrow} 0$, it is sufficient to prove that, $\forall K>2$

$$
\sup _{x_{0}}\left|\mathbb{E}\left[\mathbb{E}\left[\mathcal{H}^{n} \mathcal{X}_{K}\left(\mathcal{H}^{n}\right) \mid \bar{Y}_{1}^{n, \beta_{n}, x_{0}}\right]^{2}\right]-\mathbb{E}\left[\mathbb{E}\left[\mathcal{H}^{n} \mathcal{X}_{K}\left(\mathcal{H}^{n}\right) \mid L_{1}^{\alpha}\right]^{2}\right]\right| \stackrel{n \rightarrow \infty}{\longrightarrow} 0 .
$$

We now prove (6.26). First, we define $\eta^{\mathcal{H}^{n, K}}$ and $\bar{\eta}^{\mathcal{H}^{n, K}}$ as follows

$$
\begin{aligned}
& \eta^{\mathcal{H}^{n, K}}\left(\bar{Y}_{1}^{n, \beta_{n}, x_{0}}-\varsigma_{1}^{n, \theta_{n}, x_{0}}\right)=\mathbb{E}\left[\mathcal{H}^{n} \mathcal{X}_{K}\left(\mathcal{H}^{n}\right) \mid \bar{Y}_{1}^{n, \beta_{n}, x_{0}}-\varsigma_{1}^{n, \theta_{n}, x_{0}}\right]=\mathbb{E}\left[\mathcal{H}^{n} \mathcal{X}_{K}\left(\mathcal{H}^{n}\right) \mid \bar{Y}_{1}^{n, \beta_{n}, x_{0}}\right], \\
& \bar{\eta}^{\mathcal{H}^{n, K}}\left(\frac{\sigma L_{1}^{\alpha}}{n^{1 / \alpha}}\right)=\mathbb{E}\left[\mathcal{H}^{n} \mathcal{X}_{K}\left(\mathcal{H}^{n}\right) \mid \frac{\sigma L_{1}^{\alpha}}{n^{1 / \alpha}}\right]=\mathbb{E}\left[\mathcal{H}^{n} \mathcal{X}_{K}\left(\mathcal{H}^{n}\right) \mid L_{1}^{\alpha}\right]
\end{aligned}
$$

With these notations, we can rewrite (6.26) as

$$
\sup _{x_{0}}\left|\mathbb{E}\left[\eta^{\mathcal{H}^{n, K}}\left(\bar{Y}_{1}^{n, \beta_{n}, x_{0}}-\varsigma_{1}^{n, \theta_{n}, x_{0}}\right)^{2}\right]-\mathbb{E}\left[\bar{\eta}^{\mathcal{H}^{n, K}}\left(\frac{\sigma L_{1}^{\alpha}}{n^{1 / \alpha}}\right)^{2}\right]\right| \stackrel{n \rightarrow \infty}{\longrightarrow} 0
$$

Using Lemma 6.11 in Section 6.4, we know that:

$$
\sup _{x_{0}} \mathbb{E}\left[\left|\eta^{\mathcal{H}^{n, K}}\left(\bar{Y}_{1}^{n, \beta_{n}, x_{0}}-\varsigma_{1}^{n, \theta_{n}, x_{0}}\right)-\bar{\eta}^{\mathcal{H}^{n, K}}\left(\bar{Y}_{1}^{n, \beta_{n}, x_{0}}-\varsigma_{1}^{n, \theta_{n}, x_{0}}\right)\right|\right] \stackrel{n \rightarrow \infty}{\longrightarrow} 0
$$

and since $\left|\eta^{\mathcal{H}^{n, K}}\right|$ and $\left|\bar{\eta}^{\mathcal{H}^{n, K}}\right|$ are bounded by the constant $K$, we deduce

$$
\sup _{x_{0}}\left|\mathbb{E}\left[\eta^{\mathcal{H}^{n, K}}\left(\bar{Y}_{1}^{n, \beta_{n}, x_{0}}-\varsigma_{1}^{n, \theta_{n}, x_{0}}\right)^{2}\right]-\mathbb{E}\left[\bar{\eta}^{\mathcal{H}^{n, K}}\left(\bar{Y}_{1}^{n, \beta_{n}, x_{0}}-\varsigma_{1}^{n, \theta_{n}, x_{0}}\right)^{2}\right]\right| \stackrel{n \rightarrow \infty}{\longrightarrow} 0 .
$$

Now, applying Lemma 6.10 in Section 6.4 , with the choice $\mathcal{H}^{n}=1$ with the bounded function $\left(\bar{\eta}^{\mathcal{H}^{n, K}}\right)^{2}$ we get (6.28) and the proposition is proved.

We can now prove Lemmas 3.5-3.8.

\subsection{Proofs of Lemmas $3.5-3.8$}

Proof of Lemma 3.5. First we remark that although $L_{1}^{\alpha}$ does not belong to the domain of Malliavin operators $D$ we can establish a representation for $\varphi_{\alpha}^{\prime} / \varphi_{\alpha}$.

Indeed, since $L_{1}^{n}$ belongs to the Malliavin space $D$, the integration by parts formula (6.7) gives for any test function $f$ ( $f$ is bounded, compactly supported and $f^{\prime}$ is bounded),

$$
\mathbb{E}\left[f^{\prime}\left(L_{1}^{n}\right)\right]=\mathbb{E}\left[f\left(L_{1}^{n}\right) \mathcal{H}_{L_{1}^{n}}(1)\right]
$$

Now from Lemma 3.3, we have $\mathbb{P}\left(L_{1}^{n}=L_{1}^{\alpha}\right) \stackrel{n \rightarrow \infty}{\longrightarrow} 1$ and from Remark 6.4 ii $)$ we have $\mathcal{H}_{L_{1}^{n}}(1) \stackrel{\mathbf{L}^{p}}{\longrightarrow} \mathcal{H}_{L^{\alpha}}(1)$. Letting $n$ go to infinity in (6.29), we deduce

$$
\mathbb{E}\left[f^{\prime}\left(L_{1}^{\alpha}\right)\right]=\mathbb{E}\left[f\left(L_{1}^{\alpha}\right) \mathcal{H}_{L^{\alpha}}(1)\right]
$$

Observing that $\int \varphi_{\alpha}(u) f^{\prime}(u) \mathrm{d} u=-\int \varphi_{\alpha}^{\prime}(u) f(u) \mathrm{d} u$, we get $\int f(u) \varphi_{\alpha}^{\prime}(u) \mathrm{d} u=-\mathbb{E}\left[f\left(L_{1}^{\alpha}\right) \mathcal{H}_{L^{\alpha}}(1)\right]$ and we deduce the representation (3.7). 
Proof of Lemma 3.6. The proof of this lemma is based on the results in [6] recalled in Section 6. From (6.20), Lemma 6.5, Lemma 6.7 and (6.15), we easily deduce that $\sup _{\beta \in Q} \mathcal{R}_{\beta}^{n}\left(\nabla_{\beta} \bar{Y}_{1}^{n, \beta_{n}, x_{0}}\right)$ converges to zero in $\mathbf{L}^{p}, \forall p \geq 1$ (uniformly with respect to $x_{0}$ ).

From Lemma $6.6 i)-i i)$ and (6.19) we can deduce (3.8). The uniform control of $\mathbb{E}\left|n^{1 / 2} r_{n} \mathcal{H}_{\bar{Y}_{1}^{n, \beta, x_{0}}}\left(\nabla_{\beta} \bar{Y}_{1}^{n, \beta, x_{0}}\right)\right|^{p}$ is immediate.

Proof of Lemma 3.7. From Jensen's inequality, we have

$$
\begin{aligned}
\mathbb{E} & {\left[\left|\mathbb{E}\left[n^{1-1 / \alpha} \mathcal{H}_{\bar{Y}_{1}^{n, \beta_{n}, x_{0}}}\left(\partial_{\theta} \bar{Y}_{1}^{n, \beta_{n}, x_{0}}\right) \mid \bar{Y}_{1}^{n, \beta_{n}, x_{0}}\right]-\mathbb{E}\left[\frac{1}{\sigma} \partial_{\theta} b\left(x_{0}, \theta\right) \mathcal{H}_{L^{\alpha}}(1) \mid \bar{Y}_{1}^{n, \beta_{n}, x_{0}}\right]\right|^{2}\right] } \\
& \leq \mathbb{E}\left[\mathbb{E}\left[\left|n^{1-1 / \alpha} \mathcal{H}_{\bar{Y}_{1}^{n, \beta_{n}, x_{0}}}\left(\partial_{\theta} \bar{Y}_{1}^{n, \beta_{n}, x_{0}}\right)-\frac{1}{\sigma} \partial_{\theta} b\left(x_{0}, \theta\right) \mathcal{H}_{L^{\alpha}}(1)\right|^{2} \mid \bar{Y}_{1}^{n, \beta_{n}, x_{0}}\right]\right] \\
& =\mathbb{E}\left[\left|n^{1-1 / \alpha} \mathcal{H}_{\bar{Y}_{1}^{n, \beta_{n}, x_{0}}}\left(\partial_{\theta} \bar{Y}_{1}^{n, \beta_{n}, x_{0}}\right)-\frac{1}{\sigma} \partial_{\theta} b\left(x_{0}, \theta\right) \mathcal{H}_{L^{\alpha}}(1)\right|^{2}\right]
\end{aligned}
$$

From Lemma 3.6, the last term converges to zero uniformly with respect to $x_{0}$. In turn, it gives the uniform convergence

$$
\sup _{x_{0}}\left|n^{2-2 / \alpha} \mathbb{E}\left[\mathbb{E}\left[\mathcal{H}_{\bar{Y}_{1}^{n, \beta_{n}, x_{0}}}\left(\partial_{\theta} \bar{Y}_{1}^{n, \beta_{n}, x_{0}}\right) \mid \bar{Y}_{1}^{n, \beta_{n}, x_{0}}\right]^{2}\right]-\frac{1}{\sigma^{2}} \partial_{\theta} b\left(x_{0}, \theta\right)^{2} \mathbb{E}\left[\mathbb{E}\left[\mathcal{H}_{L^{\alpha}}(1) \mid \bar{Y}_{1}^{n, \beta_{n}, x_{0}}\right]^{2}\right]\right| \stackrel{n \rightarrow \infty}{\longrightarrow} 0 .
$$

By the same method as above, we also get the uniform convergence

$$
\sup _{x_{0}}\left|\mathbb{E}\left[\mathbb{E}\left[\mathcal{H}_{\bar{Y}_{1}^{n, \beta_{n}, x_{0}}}\left(\partial_{\sigma} \bar{Y}_{1}^{n, \beta_{n}, x_{0}}\right) \mid \bar{Y}_{1}^{n, \beta_{n}, x_{0}}\right]^{2}\right]-\frac{1}{\sigma^{2}} \mathbb{E}\left[\mathbb{E}\left[\left(L_{1}^{\alpha} \mathcal{H}_{L^{\alpha}}(1)-1\right) \mid \bar{Y}_{1}^{n, \beta_{n}, x_{0}}\right]^{2}\right]\right| \stackrel{n \rightarrow \infty}{\longrightarrow} 0 .
$$

Hence, this lemma will be proved as soon as we show that

$$
\begin{aligned}
& \sup _{x_{0}}\left|\mathbb{E}\left[\mathbb{E}\left[\mathcal{H}_{L^{\alpha}}(1) \mid \bar{Y}_{1}^{n, \beta_{n}, x_{0}}\right]^{2}\right]-\mathbb{E}\left[\mathbb{E}\left[\mathcal{H}_{L^{\alpha}}(1) \mid L_{1}^{\alpha}\right]^{2}\right]\right| \stackrel{n \rightarrow \infty}{\longrightarrow} 0, \\
& \sup _{x_{0}}\left|\mathbb{E}\left[\mathbb{E}\left[\left(L_{1}^{\alpha} \mathcal{H}_{L^{\alpha}}(1)-1\right) \mid \bar{Y}_{1}^{n, \beta_{n}, x_{0}}\right]^{2}\right]-\mathbb{E}\left[\mathbb{E}\left[\left(L_{1}^{\alpha} \mathcal{H}_{L^{\alpha}}(1)-1\right) \mid L_{1}^{\alpha}\right]^{2}\right]\right| \stackrel{n \rightarrow \infty}{\longrightarrow} 0 .
\end{aligned}
$$

To prove (6.31), we apply Proposition 6.8 with the choice $\mathcal{H}=\mathcal{H}_{L^{\alpha}}(1)$ and

$$
\mathcal{H}^{n}=\frac{\int_{0}^{1} \int_{\mathbb{R}} \rho^{n}(z)\left(\rho^{n}\right)^{\prime}(z) \mu^{n}(\mathrm{~d} s, \mathrm{~d} z)}{\left[\int_{0}^{1} \int_{\mathbb{R}} \rho^{n}(z) \mu^{n}(\mathrm{~d} s, \mathrm{~d} z)\right]^{2}}-\frac{\int_{0}^{1} \int_{\mathbb{R}}\left[\left(\rho^{n}\right)^{\prime}(z)-\frac{1+\alpha}{z} \rho^{n}(z)\right] \mu^{n}(\mathrm{~d} s, \mathrm{~d} z)}{\int_{0}^{1} \int_{\mathbb{R}} \rho^{n}(z) \mu^{n}(\mathrm{~d} s, \mathrm{~d} z)}:=\tilde{\mathcal{H}}^{n} .
$$

From Remark $6.4 i i)$ we get that $\tilde{\mathcal{H}}^{n}=\mathcal{H}_{L_{1}^{n}}(1)-\frac{1}{n^{1 / \alpha}} \mathcal{R}_{1, \beta}^{n}$, moreover $\mathbb{E}\left(\mathcal{H}_{L^{\alpha}}(1)\right)^{2}<\infty$ and $\tilde{\mathcal{H}}^{n} \stackrel{n \rightarrow \infty}{\mathbf{L}^{2}} \mathcal{H}_{L^{\alpha}}(1)$. The computation of $\Gamma\left(\tilde{\mathcal{H}}^{n}, \tilde{\mathcal{H}}^{n}\right)$ is omitted but reduces to the computation of the $\Gamma$-bracket between simple functionals. After some calculus (similar to those in the proof of Thm. 2.1 in [6]) we get that $\Gamma\left(\tilde{\mathcal{H}}^{n}, \tilde{\mathcal{H}}^{n}\right.$ ) is bounded by a random variable independent of $n$ and belonging to $\cap_{p \geq 1} \mathbf{L}^{p}$. Turning to 6.32, we proceed similarly with the choices $\mathcal{H}=L_{1}^{\alpha} \mathcal{H}_{L^{\alpha}}(1)$ and $\mathcal{H}^{n}=L_{1}^{n} \tilde{\mathcal{H}}^{n}$. Note that using Lemma 6.6 ii) with $b(x, \theta)=0$ and $\sigma=1$ we deduce that $L_{1}^{n} \tilde{\mathcal{H}}^{n} \underset{\mathbf{L}^{2}}{\stackrel{n \rightarrow \infty}{\longrightarrow}} L_{1}^{\alpha} \mathcal{H}_{L^{\alpha}}(1)$ moreover we can prove that $\sup _{n}\left\|\Gamma\left(L_{1}^{n} \tilde{\mathcal{H}}^{n}, L_{1}^{n} \tilde{\mathcal{H}}^{n}\right)\right\|_{2}<\infty$. 
Proof of Lemma 3.8. It is easy to see that

$$
\begin{aligned}
& \mathbb{E}\left[\mid n^{1-1 / \alpha} \mathbb{E}\left[\mathcal{H}_{\bar{Y}_{1}^{n, \beta_{n}, x_{0}}}\left(\partial_{\theta} \bar{Y}_{1}^{n, \beta_{n}, x_{0}}\right) \mid \bar{Y}_{1}^{n, \beta_{n}, x_{0}}\right] \mathbb{E}\left[\mathcal{H}_{\bar{Y}_{1}^{n, \beta_{n}, x_{0}}}\left(\partial_{\sigma} \bar{Y}_{1}^{n, \beta_{n}, x_{0}}\right) \mid \bar{Y}_{1}^{n, \beta_{n}, x_{0}}\right]\right. \\
& \left.-\mathbb{E}\left[\frac{1}{\sigma}\left(L_{1}^{\alpha} \mathcal{H}_{L^{\alpha}}(1)-1\right) \mid \bar{Y}_{1}^{n, \beta_{n}, x_{0}}\right] \mathbb{E}\left[\frac{1}{\sigma} \partial_{\theta} b\left(x_{0}, \theta\right) \mathcal{H}_{L^{\alpha}}(1) \mid \bar{Y}_{1}^{n, \beta_{n}, x_{0}}\right] \mid\right] \\
& \leq \mathbb{E}\left[\mid n^{1-1 / \alpha} \mathbb{E}\left[\mathcal{H}_{\bar{Y}_{1}^{n, \beta_{n}, x_{0}}}\left(\partial_{\theta} \bar{Y}_{1}^{n, \beta_{n}, x_{0}}\right) \mid \bar{Y}_{1}^{n, \beta_{n}, x_{0}}\right]\right. \\
& \left.\left(\mathbb{E}\left[\mathcal{H}_{\bar{Y}_{1}^{n, \beta_{n}, x_{0}}}\left(\partial_{\sigma} \bar{Y}_{1}^{n, \beta_{n}, x_{0}}\right) \mid \bar{Y}_{1}^{n, \beta_{n}, x_{0}}\right]-\mathbb{E}\left[\frac{\left(L_{1}^{\alpha} \mathcal{H}_{L^{\alpha}}(1)-1\right)}{\sigma} \mid \bar{Y}_{1}^{n, \beta_{n}, x_{0}}\right]\right) \mid\right] \\
& +\mathbb{E}\left[\mid \mathbb{E}\left[\frac{\left(L_{1}^{\alpha} \mathcal{H}_{L^{\alpha}}(1)-1\right)}{\sigma} \mid \bar{Y}_{1}^{n, \beta_{n}, x_{0}}\right]\right. \\
& \left.\left(n^{1-1 / \alpha} \mathbb{E}\left[\mathcal{H}_{\bar{Y}_{1}^{n, \beta_{n}, x_{0}}}\left(\partial_{\theta} \bar{Y}_{1}^{n, \beta_{n}, x_{0}}\right) \mid \bar{Y}_{1}^{n, \beta_{n}, x_{0}}\right]-\mathbb{E}\left[\frac{\partial_{\theta} b\left(x_{0}, \theta\right) \mathcal{H}_{L^{\alpha}}(1)}{\sigma} \mid \bar{Y}_{1}^{n, \beta_{n}, x_{0}}\right]\right) \mid\right] \text {. }
\end{aligned}
$$

Then using Cauchy-Schwarz inequality

$$
\begin{aligned}
& \mathbb{E}\left[\mid n^{1-1 / \alpha} \mathbb{E}\left[\mathcal{H}_{\bar{Y}_{1}^{n, \beta_{n}, x_{0}}}\left(\partial_{\theta} \bar{Y}_{1}^{n, \beta_{n}, x_{0}}\right) \mid \bar{Y}_{1}^{n, \beta_{n}, x_{0}}\right]\right. \\
& \left.\times\left(\mathbb{E}\left[\mathcal{H}_{\bar{Y}_{1}^{n, \beta_{n}, x_{0}}}\left(\partial_{\sigma} \bar{Y}_{1}^{n, \beta_{n}, x_{0}}\right) \mid \bar{Y}_{1}^{n, \beta_{n}, x_{0}}\right]-\mathbb{E}\left[\frac{\left(L_{1}^{\alpha} \mathcal{H}_{L^{\alpha}}(1)-1\right)}{\sigma} \mid \bar{Y}_{1}^{n, \beta_{n}, x_{0}}\right]\right) \mid\right] \\
& \leq\left[\mathbb{E}\left(\left(n^{1-1 / \alpha} \mathbb{E}\left[\mathcal{H}_{\bar{Y}_{1}^{n, \beta_{n}, x_{0}}}\left(\partial_{\theta} \bar{Y}_{1}^{n, \beta_{n}, x_{0}}\right) \mid \bar{Y}_{1}^{n, \beta_{n}, x_{0}}\right]\right)^{2}\right)\right]^{1 / 2} \\
& \times\left[\mathbb{E}\left(\mathbb{E}\left[\mathcal{H}_{\bar{Y}_{1}^{n, \beta_{n}, x_{0}}}\left(\partial_{\sigma} \bar{Y}_{1}^{n, \beta_{n}, x_{0}}\right) \mid \bar{Y}_{1}^{n, \beta_{n}, x_{0}}\right]-\mathbb{E}\left[\frac{\left(L_{1}^{\alpha} \mathcal{H}_{L^{\alpha}}(1)-1\right)}{\sigma} \mid \bar{Y}_{1}^{n, \beta_{n}, x_{0}}\right]\right)^{2}\right]^{1 / 2} \\
& \leq\left[\mathbb{E}\left(n^{2-2 / \alpha} \mathbb{E}\left[\left|\mathcal{H}_{\bar{Y}_{1}^{n, \beta_{n}, x_{0}}}\left(\partial_{\theta} \bar{Y}_{1}^{n, \beta_{n}, x_{0}}\right)\right|^{2} \mid \bar{Y}_{1}^{n, \beta_{n}, x_{0}}\right]\right)\right]^{1 / 2} \\
& \times\left[\mathbb{E}\left(\mathbb{E}\left[\left|\mathcal{H}_{\bar{Y}_{1}^{n, \beta_{n}, x_{0}}}\left(\partial_{\sigma} \bar{Y}_{1}^{n, \beta_{n}, x_{0}}\right)-\frac{\left(L_{1}^{\alpha} \mathcal{H}_{L^{\alpha}}(1)-1\right)}{\sigma}\right|^{2} \mid \bar{Y}_{1}^{n, \beta_{n}, x_{0}}\right]\right)\right]^{1 / 2} \\
& =\left[\mathbb{E}\left(n^{2-2 / \alpha}\left|\mathcal{H}_{\bar{Y}_{1}^{n, \beta_{n}, x_{0}}}\left(\partial_{\theta} \bar{Y}_{1}^{n, \beta_{n}, x_{0}}\right)\right|^{2}\right)\right]^{1 / 2}\left[\mathbb{E}\left(\left|\mathcal{H}_{\bar{Y}_{1}^{n, \beta_{n}, x_{0}}}\left(\partial_{\sigma} \bar{Y}_{1}^{n, \beta_{n}, x_{0}}\right)-\frac{\left(L_{1}^{\alpha} \mathcal{H}_{L^{\alpha}}(1)-1\right)}{\sigma}\right|^{2}\right)\right]^{1 / 2} .
\end{aligned}
$$


Furthermore, from (3.8) we easily deduce that (6.35) converges to zero uniformly with respect to $x_{0}$. Similarly, we also get that

$$
\mathbb{E}\left[\left|\mathbb{E}\left[\frac{\left(L_{1}^{\alpha} \mathcal{H}_{L^{\alpha}}(1)-1\right)}{\sigma} \mid \bar{Y}_{1}^{n, \beta_{n}, x_{0}}\right]\left(n^{1-1 / \alpha} \mathbb{E}\left[\mathcal{H}_{\bar{Y}_{1}^{n, \beta_{n}, x_{0}}}\left(\partial_{\theta} \bar{Y}_{1}^{n, \beta_{n}, x_{0}}\right) \mid \bar{Y}_{1}^{n, \beta_{n}, x_{0}}\right]-\mathbb{E}\left[\frac{\partial_{\theta} b\left(x_{0}, \theta\right) \mathcal{H}_{L^{\alpha}}(1)}{\sigma} \mid \bar{Y}_{1}^{n, \beta_{n}, x_{0}}\right]\right)\right|\right]
$$

tends to zero uniformly with respect to $x_{0}$. And then, we can conclude that (6.34) converges to zero uniformly with respect to $x_{0}$. In turn, it gives the uniform convergence

$$
\begin{gathered}
\sup _{x_{0}} \mid \mathbb{E}\left[n^{1-1 / \alpha} \mathbb{E}\left[\mathcal{H}_{\bar{Y}_{1}^{n, \beta_{n}, x_{0}}}\left(\partial_{\theta} \bar{Y}_{1}^{n, \beta_{n}, x_{0}}\right) \mid \bar{Y}_{1}^{n, \beta_{n}, x_{0}}\right] \mathbb{E}\left[\mathcal{H}_{\bar{Y}_{1}^{n, \beta_{n}, x_{0}}}\left(\partial_{\sigma} \bar{Y}_{1}^{n, \beta_{n}, x_{0}}\right) \mid \bar{Y}_{1}^{n, \beta_{n}, x_{0}}\right]\right]- \\
-\mathbb{E}\left[\mathbb{E}\left[\frac{1}{\sigma}\left(L_{1}^{\alpha} \mathcal{H}_{L^{\alpha}}(1)-1\right) \mid \bar{Y}_{1}^{n, \beta_{n}, x_{0}}\right] \mathbb{E}\left[\frac{1}{\sigma} \partial_{\theta} b\left(x_{0}, \theta\right) \mathcal{H}_{L^{\alpha}}(1) \mid \bar{Y}_{1}^{n, \beta_{n}, x_{0}}\right]\right] \mid \stackrel{n \rightarrow \infty}{\longrightarrow} 0 .
\end{gathered}
$$

On the other hand, we can rewrite

$$
\begin{aligned}
\mathbb{E} & {\left[\mathbb{E}\left[\frac{1}{\sigma}\left(L_{1}^{\alpha} \mathcal{H}_{L^{\alpha}}(1)-1\right) \mid \bar{Y}_{1}^{n, \beta_{n}, x_{0}}\right] \mathbb{E}\left[\frac{1}{\sigma} \partial_{\theta} b\left(x_{0}, \theta\right) \mathcal{H}_{L^{\alpha}}(1) \mid \bar{Y}_{1}^{n, \beta_{n}, x_{0}}\right]\right] } \\
& =\frac{1}{4} \mathbb{E}\left\{\left[\mathbb{E}\left(\frac{1}{\sigma}\left(L_{1}^{\alpha} \mathcal{H}_{L^{\alpha}}(1)-1\right)+\frac{1}{\sigma} \partial_{\theta} b\left(x_{0}, \theta\right) \mathcal{H}_{L^{\alpha}}(1) \mid \bar{Y}_{1}^{n, \beta_{n}, x_{0}}\right)\right]^{2}\right. \\
& \left.-\left[\mathbb{E}\left(\frac{1}{\sigma}\left(L_{1}^{\alpha} \mathcal{H}_{L^{\alpha}}(1)-1\right)-\frac{1}{\sigma} \partial_{\theta} b\left(x_{0}, \theta\right) \mathcal{H}_{L^{\alpha}}(1) \mid \bar{Y}_{1}^{n, \beta_{n}, x_{0}}\right)\right]^{2}\right\} .
\end{aligned}
$$

Then, the lemma will be proved as soon as we show that

$$
\begin{gathered}
\mathbb{E}\left\{\left[\mathbb{E}\left(\frac{1}{\sigma}\left(L_{1}^{\alpha} \mathcal{H}_{L^{\alpha}}(1)-1\right)+\frac{1}{\sigma} \partial_{\theta} b\left(x_{0}, \theta\right) \mathcal{H}_{L^{\alpha}}(1) \mid \bar{Y}_{1}^{n, \beta_{n}, x_{0}}\right)\right]^{2}\right. \\
\left.-\left[\mathbb{E}\left(\frac{1}{\sigma}\left(L_{1}^{\alpha} \mathcal{H}_{L^{\alpha}}(1)-1\right)-\frac{1}{\sigma} \partial_{\theta} b\left(x_{0}, \theta\right) \mathcal{H}_{L^{\alpha}}(1) \mid \bar{Y}_{1}^{n, \beta_{n}, x_{0}}\right)\right]^{2}\right\}
\end{gathered}
$$

is uniformly convergent with respect to $x_{0}$ to

$$
\begin{gathered}
\mathbb{E}\left\{\left[\mathbb{E}\left(\frac{1}{\sigma}\left(L_{1}^{\alpha} \mathcal{H}_{L^{\alpha}}(1)-1\right)+\frac{1}{\sigma} \partial_{\theta} b\left(x_{0}, \theta\right) \mathcal{H}_{L^{\alpha}}(1) \mid L_{1}^{\alpha}\right)\right]^{2}\right. \\
\left.-\left[\mathbb{E}\left(\frac{1}{\sigma}\left(L_{1}^{\alpha} \mathcal{H}_{L^{\alpha}}(1)-1\right)-\frac{1}{\sigma} \partial_{\theta} b\left(x_{0}, \theta\right) \mathcal{H}_{L^{\alpha}}(1) \mid L_{1}^{\alpha}\right)\right]^{2}\right\} .
\end{gathered}
$$

We end the proof by using Proposition 6.8 with $\mathcal{H}=L_{1}^{\alpha} \mathcal{H}_{L^{\alpha}}(1) \pm \partial_{\theta} b\left(x_{0}, \theta\right) \mathcal{H}_{L^{\alpha}}(1)$ and $\mathcal{H}^{n}=L_{1}^{n} \tilde{\mathcal{H}}^{n} \pm$ $\partial_{\theta} b\left(x_{0}, \theta\right) \tilde{\mathcal{H}}^{n}$ where $\tilde{\mathcal{H}}^{n}$ is given by $(6.33)$. 


\subsection{Lemmas 6.10 and 6.11}

The aim of this section is to show that the functions $\eta^{\mathcal{H}^{n, K}}$ and $\bar{\eta}^{\mathcal{H}^{n, K}}$ defined by (6.27) are close in some sense. The idea is mainly based on ([5], Prop. 9, p. 2348), however we need a more technical study since $\alpha \in(0,2)$ and the function $b$ is not assumed to be bounded. Our first result is the following.

Lemma 6.10. Under the assumptions of Proposition 6.8, for all bounded function $h, \forall K>2$, there exists a constant $C_{K}>0$ such that

$$
\left|\mathbb{E}\left[\mathcal{H}^{n} \mathcal{X}_{K}\left(\mathcal{H}^{n}\right) h\left(\bar{Y}_{1}^{n, \beta, x_{0}}-\varsigma_{1}^{n, \theta, x_{0}}\right)\right]-\mathbb{E}\left[\mathcal{H}^{n} \mathcal{X}_{K}\left(\mathcal{H}^{n}\right) h\left(\frac{\sigma L_{1}^{\alpha}}{n^{1 / \alpha}}\right)\right]\right| \leq \frac{C_{K}}{n}\|h\|_{\infty}
$$

and the above estimate is uniform with respect to $x_{0} \in \mathbb{R}$ and $\beta \in Q$, for any compact set $Q \subset \mathbb{R} \times(0, \infty)$.

Proof. Since $\mathcal{H}^{n} \mathcal{X}_{K}\left(\mathcal{H}^{n}\right)$ is bounded and $\mathbb{P}\left(L_{1}^{n} \neq L_{1}^{\alpha}\right) \leq \frac{C}{n}$ (see Lem. 3.3) it is sufficient to show that

$$
\left|\mathbb{E}\left[\mathcal{H}^{n} \mathcal{X}_{K}\left(\mathcal{H}^{n}\right) h\left(\bar{Y}_{1}^{n, \beta, x_{0}}-\varsigma_{1}^{n, \theta, x_{0}}\right)\right]-\mathbb{E}\left[\mathcal{H}^{n} \mathcal{X}_{K}\left(\mathcal{H}^{n}\right) h\left(\frac{\sigma L_{1}^{n}}{n^{1 / \alpha}}\right)\right]\right| \leq \frac{C_{K}}{n}\|h\|_{\infty}
$$

We now prove $(6.40)$.

Let us denote $\mathcal{H}^{n, K}=\mathcal{H}^{n} \mathcal{X}_{K}\left(\mathcal{H}^{n}\right)$ and $H$ any primitive function of $h$. Using the integration by parts formula (6.7), we have

$$
\mathbb{E}\left[h\left(\frac{\sigma L_{1}^{n}}{n^{1 / \alpha}}\right) \mathcal{H}^{n, K}\right]=\mathbb{E}\left[H\left(\frac{\sigma L_{1}^{n}}{n^{1 / \alpha}}\right) \mathcal{H}_{\left(\frac{\sigma L_{1}^{n}}{n^{1 / \alpha}}\right)}\left(\mathcal{H}^{n, K}\right)\right]
$$

where $\mathcal{H}_{\left(\frac{\sigma L_{1}^{n}}{n^{1 / \alpha}}\right)}\left(\mathcal{H}^{n, K}\right)$ is given by $(6.10)$, namely here

$$
\mathcal{H}_{\left(\frac{\sigma L_{1}^{n}}{n^{1 / \alpha}}\right)}\left(\mathcal{H}^{n, K}\right)=L\left(\frac{\mathcal{H}^{n, K}}{\Gamma\left(\frac{\sigma L_{1}^{n}}{n^{1 / \alpha}}, \frac{\sigma L_{1}^{n}}{n^{1 / \alpha}}\right)}\right) \frac{\sigma L_{1}^{n}}{n^{1 / \alpha}}-\frac{L\left(\frac{\sigma L_{1}^{n}}{n^{1 / \alpha}}\right) \mathcal{H}^{n, K}}{\Gamma\left(\frac{\sigma L_{1}^{n}}{n^{1 / \alpha}}, \frac{\sigma L_{1}^{n}}{n^{1 / \alpha}}\right)}-L\left(\frac{\mathcal{H}^{n, K} \frac{\sigma L_{1}^{n}}{n^{1 / \alpha}}}{\Gamma\left(\frac{\sigma L_{1}^{n}}{n^{1 / \alpha}}, \frac{\sigma L_{1}^{n}}{n^{1 / \alpha}}\right)}\right) .
$$

On the other hand, we have for $t \in[0,1]$

$$
\begin{aligned}
\left|\bar{Y}_{t}^{n, \beta, x_{0}}-\varsigma_{t}^{n, \theta, x_{0}}-\frac{\sigma L_{t}^{n}}{n^{1 / \alpha}}\right| & =\left|\frac{1}{n} \int_{0}^{t}\left[b\left(\bar{Y}_{s}^{n, \beta, x_{0}}, \theta\right)-b\left(\varsigma_{s}^{n, \theta, x_{0}}, \theta\right)\right] \mathrm{d} s\right| \\
& \leq \frac{1}{n} \int_{0}^{t}|| b^{\prime} \|_{\infty}\left|\bar{Y}_{s}^{n, \beta, x_{0}}-\varsigma_{s}^{n, \theta, x_{0}}\right| \mathrm{d} s \\
& \leq \frac{1}{n} \int_{0}^{t}\left\|b^{\prime}\right\|_{\infty}\left|\bar{Y}_{s}^{n, \beta, x_{0}}-\varsigma_{s}^{n, \theta, x_{0}}-\frac{\sigma L_{s}^{n}}{n^{1 / \alpha}}\right| \mathrm{d} s+\frac{1}{n^{1+1 / \alpha}} \int_{0}^{1}\left|\sigma L_{s}^{n}\right| \mathrm{d} s
\end{aligned}
$$

Applying the Gronwall's inequality, for $C$ a positive constant, independent of $n$ and $K$,

$$
\left|\bar{Y}_{1}^{n, \beta, x_{0}}-\varsigma_{1}^{n, \theta, x_{0}}-\frac{\sigma L_{1}^{n}}{n^{1 / \alpha}}\right| \leq \frac{C}{n^{1+1 / \alpha}} \int_{0}^{1}\left|\sigma L_{s}^{n}\right| \mathrm{d} s
$$

Using that the function $H$ is globally Lipschitz with a Lipschitz constant $\|h\|_{\infty}$, we deduce from (6.41) that

$$
\left|\mathbb{E}\left[\mathcal{H}^{n, K} h\left(\frac{\sigma L_{1}^{n}}{n^{1 / \alpha}}\right)\right]-\mathbb{E}\left[H\left(\bar{Y}_{1}^{n, \beta, x_{0}}-\varsigma_{1}^{n, \theta, x_{0}}\right) \mathcal{H}_{\left(\frac{\sigma L_{1}^{n}}{n^{1 / \alpha}}\right)}\left(\mathcal{H}^{n, K}\right)\right]\right|
$$




$$
\leq\left|\frac{\sigma C}{n^{1+1 / \alpha}}\right|\|h\|_{\infty} \mathbb{E}\left[\left|\int_{0}^{1}\right| L_{s}^{n}\left|\mathrm{~d} s \mathcal{H}_{\left(\frac{\sigma L_{1}^{n}}{n^{1 / \alpha}}\right)}\left(\mathcal{H}^{n, K}\right)\right|\right] .
$$

Now we compute $\mathbb{E}\left[H\left(\bar{Y}_{1}^{n, \beta, x_{0}}-\varsigma_{1}^{n, \theta, x_{0}}\right) \mathcal{H}_{\left(\frac{\sigma L_{1}^{n}}{n^{1 / \alpha}}\right)}\left(\mathcal{H}^{n, K}\right)\right]$ using successively the self-adjoint property of the operator $L,(6.3)$ and (6.10), to obtain an integration by part formula in a reverse direction:

$$
\begin{aligned}
& \mathbb{E}\left[H\left(\bar{Y}_{1}^{n, \beta, x_{0}}-\varsigma_{1}^{n, \theta, x_{0}}\right) \mathcal{H}_{\left(\frac{\sigma L_{1}^{n}}{n^{1 / \alpha}}\right)}\left(\mathcal{H}^{n, K}\right)\right] \\
& =\mathbb{E}\left[H\left(\bar{Y}_{1}^{n, \beta, x_{0}}-\varsigma_{1}^{n, \theta, x_{0}}\right)\left\{L\left(\frac{\mathcal{H}^{n, K}}{\Gamma\left(\frac{\sigma L_{1}^{n}}{n^{1 / \alpha}}, \frac{\sigma L_{1}^{n}}{n^{1 / \alpha}}\right)}\right) \frac{\sigma L_{1}^{n}}{n^{1 / \alpha}}-\frac{L\left(\frac{\sigma L_{1}^{n}}{n^{1 / \alpha}}\right) \mathcal{H}^{n, K}}{\Gamma\left(\frac{\sigma L_{1}^{n}}{n^{1 / \alpha}}, \frac{\sigma L_{1}^{n}}{n^{1 / \alpha}}\right)}-L\left(\frac{\mathcal{H}^{n, K} \frac{\sigma L_{1}^{n}}{n^{1 / \alpha}}}{\Gamma\left(\frac{\sigma L_{1}^{n}}{n^{1 / \alpha}}, \frac{\sigma L_{1}^{n}}{n^{1 / \alpha}}\right)}\right)\right\}\right] \\
& =\mathbb{E}\left[\frac{\left.\left\{L\left(H\left(\bar{Y}_{1}^{n, \beta, x_{0}}-\varsigma_{1}^{n, \theta, x_{0}}\right) \frac{\sigma L_{1}^{n}}{n^{1 / \alpha}}\right)-H\left(\bar{Y}_{1}^{n, \beta, x_{0}}-\varsigma_{1}^{n, \theta, x_{0}}\right) L\left(\frac{\sigma L_{1}^{n}}{n^{1 / \alpha}}\right)-L\left(H\left(\bar{Y}_{1}^{n, \beta, x_{0}}-\varsigma_{1}^{n, \theta, x_{0}}\right)\right) \frac{\sigma L_{1}^{n}}{n^{1 / \alpha}}\right)\right\} \mathcal{H}^{n, K}}{\Gamma\left(\frac{\sigma L_{1}^{n}}{n^{1 / \alpha}}, \frac{\sigma L_{1}^{n}}{n^{1 / \alpha}}\right)}\right]=\mathbb{E}\left[\mathcal{H}^{n, K} h\left(\bar{Y}_{1}^{n, \beta, x_{0}}-\varsigma_{1}^{n, \theta, x_{0}}\right) \frac{\Gamma\left(\frac{\sigma L_{1}^{n}}{n^{1 / \alpha}}, \bar{Y}_{1}^{n, \beta, x_{0}}-\varsigma_{1}^{n, \theta, x_{0}}\right)}{\Gamma\left(\frac{\sigma L_{1}^{n}}{n^{1 / \alpha}}, \frac{\sigma L_{1}^{n}}{n^{1 / \alpha}}\right)}\right] . \\
& =\mathbb{E}\left[\mathcal{H}^{n, K} \frac{\Gamma\left(\frac{\sigma L_{1}^{n}}{n^{1 / \alpha}}, H\left(\bar{Y}_{1}^{n, \beta, x_{0}}-\varsigma_{1}^{n, \theta, x_{0}}\right)\right)}{\Gamma\left(\frac{\sigma L_{1}^{n}}{n^{1 / \alpha}}, \frac{\sigma L_{1}^{n}}{n^{1 / \alpha}}\right)}\right]
\end{aligned}
$$

Putting together (6.43) and (6.44) we deduce,

$$
\begin{aligned}
& \left|\mathbb{E}\left[\mathcal{H}^{n, K} h\left(\frac{\sigma L_{1}^{n}}{n^{1 / \alpha}}\right)\right]-\mathbb{E}\left[\mathcal{H}^{n, K} h\left(\bar{Y}_{1}^{n, \beta, x_{0}}-\varsigma_{1}^{n, \theta, x_{0}}\right)\right] \leq\left(\frac{\sigma C}{n^{1+1 / \alpha}}\right)\|h\|_{\infty}\left\|\mathcal{H}_{\left(\frac{\sigma L_{1}^{n}}{n^{1 / \alpha}}\right)}\left(\mathcal{H}^{n, K}\right) \int_{0}^{1}\left|L_{s}^{n}\right| \mathrm{d} s\right\|_{1}\right. \\
& \quad+\mathbb{E}\left[\left|\mathcal{H}^{n, K} h\left(\bar{Y}_{1}^{n, \beta, x_{0}}-\varsigma_{1}^{n, \theta, x_{0}}\right)\left\{\frac{\Gamma\left(\frac{\sigma L_{1}^{n}}{n^{1 / \alpha}}, \bar{Y}_{1}^{n, \beta, x_{0}}-\varsigma_{1}^{n, \theta, x_{0}}\right)}{\Gamma\left(\frac{\sigma L_{1}^{n}}{n^{1 / \alpha}}, \frac{\sigma L_{1}^{n}}{n^{1 / \alpha}}\right)}-1\right\}\right|\right] \\
& \leq\left(\frac{\sigma C}{n^{1+1 / \alpha}}\right)\|h\|_{\infty}\left\|\mathcal{H}_{\left(\frac{\sigma L_{1}^{n}}{n^{1 / \alpha}}\right)}\left(\mathcal{H}^{n, K}\right) \int_{0}^{1}\left|L_{s}^{n}\right| \mathrm{d} s\right\|_{1}+\left\|\mathcal{H}^{n, K}\right\|_{\infty}\|h\|_{\infty}\left\|\frac{\Gamma\left(\frac{\sigma L_{1}^{n}}{n^{1 / \alpha}}, \bar{Y}_{1}^{n, \beta, x_{0}}-\varsigma_{1}^{n, \theta, x_{0}}\right)}{\Gamma\left(\frac{\sigma L_{1}^{n}}{n^{1 / \alpha}}, \frac{\sigma L_{1}^{n}}{n^{1 / \alpha}}\right)}-1\right\|_{1} \\
& \leq \frac{C}{n}\|h\|_{\infty} \int_{0}^{1} \underbrace{\left\|\frac{\sigma}{n^{1 / \alpha}} \mathcal{H}_{\left(\frac{\sigma L_{1}^{n}}{n^{1 / \alpha}}\right)}\left(\mathcal{H}^{n, K}\right)\left|L_{s}^{n}\right|\right\|_{1}}_{I_{1}^{(n, s)}} \mathrm{d} s+\left\|\mathcal{H}^{n, K}\right\|_{\infty}\|h\|_{\infty} \underbrace{\left\|\frac{\Gamma\left(\frac{\sigma L_{1}^{n}}{n^{1 / \alpha}}, \bar{Y}_{1}^{n, \beta, x_{0}}-\varsigma_{1}^{n, \theta, x_{0}}\right)}{\Gamma\left(\frac{\sigma L_{1}^{n}}{n^{1 / \alpha}}, \frac{\sigma L_{1}^{n}}{n^{1 / \alpha}}\right)}-1\right\|_{1}}_{I_{2}^{n}} .
\end{aligned}
$$

Hence the lemma will be proved if we show that $\sup _{n} \sup _{s \in[0,1]} I_{1}^{(n, s)}<\infty$ and $\sup _{n} n I_{2}^{(n)}<\infty$.

Step 1: we show that $\sup _{n} \sup _{s \in[0,1]} I_{1}^{(n, s)}<\infty$.

We can write from (6.9)

$$
\begin{aligned}
\mathcal{H}_{\left(\frac{\sigma L_{1}^{n}}{n^{1 / \alpha}}\right)}\left(\mathcal{H}^{n, K}\right) & =\frac{-2 L\left(\frac{\sigma L_{1}^{n}}{n^{1 / \alpha}}\right) \mathcal{H}^{n, K}}{\Gamma\left(\frac{\sigma L_{1}^{n}}{n^{1 / \alpha}}, \frac{\sigma L_{1}^{n}}{n^{1 / \alpha}}\right)}+\frac{\mathcal{H}^{n, K}}{\Gamma\left(\frac{\sigma L_{1}^{n}}{n^{1 / \alpha}}, \frac{\sigma L_{1}^{n}}{n^{1 / \alpha}}\right)^{2}} \Gamma\left(\frac{\sigma L_{1}^{n}}{n^{1 / \alpha}}, \Gamma\left(\frac{\sigma L_{1}^{n}}{n^{1 / \alpha}}, \frac{\sigma L_{1}^{n}}{n^{1 / \alpha}}\right)\right)-\frac{\Gamma\left(\frac{\sigma L_{1}^{n}}{n^{1 / \alpha}}, \mathcal{H}^{n, K}\right)}{\Gamma\left(\frac{\sigma L_{1}^{n}}{n^{1 / \alpha}}, \frac{\sigma L_{1}^{n}}{n^{1 / \alpha}}\right)} \\
& =\frac{n^{1 / \alpha}}{\sigma}\left[\frac{-2 L\left(L_{1}^{n}\right) \mathcal{H}^{n, K}}{\Gamma\left(L_{1}^{n}, L_{1}^{n}\right)}+\frac{\mathcal{H}^{n, K}}{\Gamma\left(L_{1}^{n}, L_{1}^{n}\right)^{2}} \Gamma\left(L_{1}^{n}, \Gamma\left(L_{1}^{n}, L_{1}^{n}\right)\right)-\frac{\Gamma\left(L_{1}^{n}, \mathcal{H}^{n, K}\right)}{\Gamma\left(L_{1}^{n}, L_{1}^{n}\right)}\right] .
\end{aligned}
$$

Now, let us recall that from (3.2)

$$
L_{s}^{n}=\int_{0}^{s} \int_{|z| \leq 1} z \tilde{\mu}^{(n)}(\mathrm{d} t, \mathrm{~d} z)+\int_{0}^{s} \int_{|z|>1} z \mu^{(n)}(\mathrm{d} t, \mathrm{~d} z),
$$


then,

$$
\begin{aligned}
\left|\frac{\sigma}{n^{1 / \alpha}} \mathcal{H}_{\left(\frac{\sigma L_{1}^{n}}{n^{1 / \alpha}}\right)}\left(\mathcal{H}^{n, K}\right)\right| L_{s}^{n}|| \leq & \left|\frac{\sigma}{n^{1 / \alpha}} \mathcal{H}_{\left(\frac{\sigma L_{1}^{n}}{n^{1 / \alpha}}\right)}\left(\mathcal{H}^{n, K}\right) \int_{0}^{s} \int_{|z| \leq 1} z \tilde{\mu}^{(n)}(\mathrm{d} t, \mathrm{~d} z)\right| \\
& +\left|\frac{\sigma}{n^{1 / \alpha}} \mathcal{H}_{\left(\frac{\sigma L_{1}^{n}}{n^{1 / \alpha}}\right)}\left(\mathcal{H}^{n, K}\right) \int_{0}^{1} \int_{|z|>1}\right| z\left|\mu^{(n)}(\mathrm{d} t, \mathrm{~d} z)\right|
\end{aligned}
$$

First, we consider the expectation of the right-hand side term in (6.47), we have:

$$
\begin{aligned}
& \mathbb{E}\left[\left|{\frac{\sigma}{n^{1 / \alpha}}}_{\left(\frac{\sigma L_{1}^{n}}{\left.n^{1 / \alpha}\right)}\right.}\left(\mathcal{H}^{n, K}\right) \int_{0}^{s} \int_{|z| \leq 1} z \tilde{\mu}^{(n)}(\mathrm{d} t, \mathrm{~d} z)\right|\right] \\
& \leq\left[\mathbb{E}\left(\int_{0}^{s} \int_{|z| \leq 1} z \tilde{\mu}^{(n)}(\mathrm{d} t, \mathrm{~d} z)\right)^{2}\right]^{1 / 2}\left[\mathbb{E}\left(\frac{\sigma}{n^{1 / \alpha}} \mathcal{H}_{\left(\frac{\sigma L_{1}^{n}}{n^{1 / \alpha}}\right)}\left(\mathcal{H}^{n, K}\right)\right)^{2}\right]^{1 / 2} \text { by Cauchy-Schwarz inequality, } \\
& =\left[\int_{0}^{1} \int_{|z| \leq 1} z^{2} \frac{1}{|z|^{1+\alpha}} \mathrm{d} z \mathrm{~d} t\right]^{1 / 2}\left[\mathbb{E}\left(\frac{\sigma}{n^{1 / \alpha}} \mathcal{H}_{\left(\frac{\sigma L_{1}^{n}}{n^{1 / \alpha}}\right)}\left(\mathcal{H}^{n, K}\right)\right)^{2}\right]^{1 / 2} \leq M\left[\mathbb{E}\left(\frac{\sigma}{n^{1 / \alpha}} \mathcal{H}_{\left(\frac{\sigma L_{1}^{n}}{n^{1 / \alpha}}\right)}\left(\mathcal{H}^{n, K}\right)\right)^{2}\right]^{1 / 2}
\end{aligned}
$$

where $M$ is a deterministic constant.

Furthermore, from (6.6) we have $\left|\Gamma\left(L_{1}^{n}, \mathcal{H}^{n, K}\right)\right| \leq \Gamma\left(\mathcal{H}^{n, K}, \mathcal{H}^{n, K}\right)^{1 / 2} \Gamma\left(L_{1}^{n}, L_{1}^{n}\right)^{1 / 2}$ and from (6.46), convexity inequality, we get

$$
\left[\left|\frac{\sigma}{n^{1 / \alpha}} \mathcal{H}_{\left(\frac{\sigma L_{1}^{n}}{\left.n^{1 / \alpha}\right)}\right.}\left(\mathcal{H}^{n, K}\right)\right|\right]^{2} \leq 2\left[\left(\frac{-2 L\left(L_{1}^{n}\right) \mathcal{H}^{n, K}}{\Gamma\left(L_{1}^{n}, L_{1}^{n}\right)}+\frac{\mathcal{H}^{n, K}}{\Gamma\left(L_{1}^{n}, L_{1}^{n}\right)^{2}} \Gamma\left(L_{1}^{n}, \Gamma\left(L_{1}^{n}, L_{1}^{n}\right)\right)\right)^{2}+\frac{\Gamma\left(\mathcal{H}^{n, K}, \mathcal{H}^{n, K}\right)}{\Gamma\left(L_{1}^{n}, L_{1}^{n}\right)}\right] .
$$

Then, we can deduce that

$$
\mathbb{E}\left(\left|\frac{\sigma}{n^{1 / \alpha}} \mathcal{H}_{\left(\frac{\sigma L_{1}^{n}}{n^{1 / \alpha}}\right)}\left(\mathcal{H}^{n, K}\right)\right|\right)^{2} \leq 2 \mathbb{E} \underbrace{\left[\left(\frac{-2 L\left(L_{1}^{n}\right) \mathcal{H}^{n, K}}{\Gamma\left(L_{1}^{n}, L_{1}^{n}\right)}+\frac{\mathcal{H}^{n, K}}{\Gamma\left(L_{1}^{n}, L_{1}^{n}\right)^{2}} \Gamma\left(L_{1}^{n}, \Gamma\left(L_{1}^{n}, L_{1}^{n}\right)\right)\right)^{2}\right]}_{I_{1.1}^{n}}+2 \underbrace{\mathbb{E}\left[\frac{\Gamma\left(\mathcal{H}^{n, K}, \mathcal{H}^{n, K}\right)}{\Gamma\left(L_{1}^{n}, L_{1}^{n}\right)}\right]}_{I_{1.2}^{n}} .
$$

Our aim is to prove that $I_{1.1}^{n}$ and $I_{1.2}^{n}$ are bounded independently of $n$.

For $I_{1.1}^{n}$, we see from (6.8) and Remark 6.4,

$$
\frac{\Gamma\left(L_{1}^{n}, \Gamma\left(L_{1}^{n}, L_{1}^{n}\right)\right)}{\Gamma\left(L_{1}^{n}, L_{1}^{n}\right)^{2}}-\frac{2 L\left(L_{1}^{n}\right)}{\Gamma\left(L_{1}^{n}, L_{1}^{n}\right)}=\mathcal{H}_{L_{1}^{n}}(1)=\widehat{\mathcal{H}}_{\beta}^{n}(1)+\frac{1}{n^{1 / \alpha}} \mathcal{R}_{1, \beta}^{n} .
$$

From the crucial fact $\left\|\mathcal{H}^{n, K}\right\|_{\infty} \leq K$ and from (6.12), (6.15), Remark 6.4 we can deduce that $I_{1.1}^{n}$ is bounded by a random variable independent of $n$ (but depending on $K$ ).

For $I_{1.2}^{n}$, from (6.1) and (6.21), we have

$$
I_{1.2}^{n}=\mathbb{E}\left(\frac{\Gamma\left(\mathcal{H}^{n, K}, \mathcal{H}^{n, K}\right)}{\Gamma\left(L_{1}^{n}, L_{1}^{n}\right)}\right)=\mathbb{E}\left(\frac{\Gamma\left(\mathcal{H}^{n, K}, \mathcal{H}^{n, K}\right)}{\int_{0}^{1} \int_{\mathbb{R}} \rho^{n}(z) \mu^{(n)}(\mathrm{d} t, \mathrm{~d} z)}\right) \leq \mathbb{E}\left(\frac{\Gamma\left(\mathcal{H}^{n, K}, \mathcal{H}^{n, K}\right)}{\int_{0}^{1} \int_{|z|<1} z^{4} \mu(\mathrm{d} t, \mathrm{~d} z)}\right) .
$$

Now since $\mathcal{H}^{n, K}$ is a smooth Malliavin functional, using the chain rule property (6.4) we have

$$
\Gamma\left(\mathcal{H}^{n, K}, \mathcal{H}^{n, K}\right) \leq c_{K}^{2} \Gamma\left(\mathcal{H}^{n}, \mathcal{H}^{n}\right)
$$


where $c_{K}$ is any upper bound of the derivative of $x \mapsto x \mathcal{X}_{K}(x)$.

Then we deduce that

$$
I_{1.2}^{n} \leq c_{K}^{2} \mathbb{E}\left(\frac{\Gamma\left(\mathcal{H}^{n}, \mathcal{H}^{n}\right)}{\int_{0}^{1} \int_{|z|<1} z^{4} \mu(\mathrm{d} t, \mathrm{~d} z)}\right)
$$

From the assumption on $\Gamma\left(\mathcal{H}^{n}, \mathcal{H}^{n}\right)$ in Proposition 6.8 and since $\left(\int_{0}^{1} \int_{|z|<1} z^{4} \mu(\mathrm{d} t, \mathrm{~d} z)\right)^{-1}$ belongs to $\cap_{p \geq 1} \mathbf{L}^{p}$ (see [5], the proof of Thm. 4), we can deduce that $I_{1.2}^{n}$ is bounded independently of $n$. Thus, we get that the expectation of the right-hand side term in (6.47) is finite.

Turning to the expectation of (6.48), we have:

$$
\begin{aligned}
& \mathbb{E}\left[\left|\frac{\sigma}{n^{1 / \alpha}} \mathcal{H}_{\left(\frac{\sigma L_{1}^{n}}{n^{1 / \alpha}}\right)}\left(\mathcal{H}^{n, K}\right) \int_{0}^{1} \int_{|z|>1}\right| z\left|\mu^{(n)}(\mathrm{d} t, \mathrm{~d} z)\right|\right] \\
& =\mathbb{E}\left[\left|\frac{\sigma}{n^{1 / \alpha}} \mathcal{H}_{\left(\frac{\sigma L_{1}^{n}}{n^{1 / \alpha}}\right)}\left(\mathcal{H}^{n, K}\right)\left(\int_{0}^{1} \int_{1 \leq|z| \leq 2}|z| \mu^{(n)}(\mathrm{d} t, \mathrm{~d} z)+\int_{0}^{1} \int_{|z|>2}|z| \mu^{(n)}(\mathrm{d} t, \mathrm{~d} z)\right)\right|\right] \\
& \leq \mathbb{E}\left[\left|\frac{\sigma}{n^{1 / \alpha}} \mathcal{H}_{\left(\frac{\sigma L_{1}^{n}}{n^{1 / \alpha}}\right)}\left(\mathcal{H}^{n, K}\right) \int_{0}^{1} \int_{1 \leq|z| \leq 2}\right| z\left|\mu^{(n)}(\mathrm{d} t, \mathrm{~d} z)\right|\right]+\mathbb{E}\left[\left|\frac{\sigma}{n^{1 / \alpha}} \mathcal{H}_{\left(\frac{\sigma L_{1}^{n}}{n^{1 / \alpha}}\right)}\left(\mathcal{H}^{n, K}\right) \int_{0}^{1} \int_{|z|>2}\right| z\left|\mu^{(n)}(\mathrm{d} t, \mathrm{~d} z)\right|\right] .
\end{aligned}
$$

By a similar estimation technique as for the bound of (6.47), we get that

$$
\sup _{n} \mathbb{E}\left[\left|\frac{\sigma}{n^{1 / \alpha}} \mathcal{H}_{\left(\frac{\sigma L_{1}^{n}}{n^{1 / \alpha}}\right)}\left(\mathcal{H}^{n, K}\right) \int_{0}^{1} \int_{1 \leq|z| \leq 2}\right| z\left|\mu^{(n)}(\mathrm{d} t, \mathrm{~d} z)\right|\right] \leq C_{K}<+\infty .
$$

We now show that

$$
\sup _{n} \mathbb{E}\left[\left|\frac{\sigma}{n^{1 / \alpha}} \mathcal{H}_{\left(\frac{\sigma L_{1}^{n}}{n^{1 / \alpha}}\right)}\left(\mathcal{H}^{n, K}\right) \int_{0}^{1} \int_{|z|>2}\right| z\left|\mu^{(n)}(\mathrm{d} t, \mathrm{~d} z)\right|\right] \leq C_{K}<+\infty .
$$

In fact, from (6.46) we have

$$
\begin{aligned}
& \mathbb{E}\left[\left|\frac{\sigma}{n^{1 / \alpha}} \mathcal{H}_{\left(\frac{\sigma L_{1}^{n}}{n^{1 / \alpha}}\right)}\left(\mathcal{H}^{n, K}\right) \int_{0}^{1} \int_{|z|>2}\right| z\left|\mu^{(n)}(\mathrm{d} t, \mathrm{~d} z)\right|\right] \\
& \left.\leq \mathbb{E}\left[|| \frac{-2 L\left(L_{1}^{n}\right) \mathcal{H}^{n, K}}{\Gamma\left(L_{1}^{n}, L_{1}^{n}\right)}+\frac{\mathcal{H}^{n, K}}{\Gamma\left(L_{1}^{n}, L_{1}^{n}\right)^{2}} \Gamma\left(L_{1}^{n}, \Gamma\left(L_{1}^{n}, L_{1}^{n}\right)\right)|+| \frac{\Gamma\left(L_{1}^{n}, \mathcal{H}^{n, K}\right)}{\Gamma\left(L_{1}^{n}, L_{1}^{n}\right)} \mid\right] \times \int_{0}^{1} \int_{|z|>2}|z| \mu^{(n)}(\mathrm{d} t, \mathrm{~d} z)\right] \\
& = \\
& \underbrace{\mathbb{E}\left[\left|\frac{-2 L\left(L_{1}^{n}\right) \mathcal{H}^{n, K}}{\Gamma\left(L_{1}^{n}, L_{1}^{n}\right)}+\frac{\mathcal{H}^{n, K}}{\Gamma\left(L_{1}^{n}, L_{1}^{n}\right)^{2}} \Gamma\left(L_{1}^{n}, \Gamma\left(L_{1}^{n}, L_{1}^{n}\right)\right)\right| \int_{0}^{1} \int_{|z|>2}|z| \mu^{(n)}(\mathrm{d} t, \mathrm{~d} z)\right]}_{I_{1.3}^{n}} \\
& +\underbrace{\mathbb{E}\left[\left|\frac{\Gamma\left(L_{1}^{n}, \mathcal{H}^{n, K}\right)}{\Gamma\left(L_{1}^{n}, L_{1}^{n}\right)}\right| \int_{0}^{1} \int_{|z|>2}|z| \mu^{(n)}(\mathrm{d} t, \mathrm{~d} z)\right]}_{I_{1.4}^{n}}
\end{aligned}
$$


To prove (6.54), we just have to show that $I_{1.3}^{n}$ and $I_{1.4}^{n}$ are bounded independently of $n$. For $I_{1.3}^{n}$, we recall here (6.52),

$$
\frac{\Gamma\left(L_{1}^{n}, \Gamma\left(L_{1}^{n}, L_{1}^{n}\right)\right)}{\Gamma\left(L_{1}^{n}, L_{1}^{n}\right)^{2}}-\frac{2 L\left(L_{1}^{n}\right)}{\Gamma\left(L_{1}^{n}, L_{1}^{n}\right)}=\widehat{\mathcal{H}}_{\beta}^{n}(1)+\frac{1}{n^{1 / \alpha}} \mathcal{R}_{1, \beta}^{n}(1)
$$

Then from the boundedness of $\mathcal{H}^{n, K}$, we get

$$
I_{1.3}^{n} \leq K \mathbb{E}\left[\left|\widehat{\mathcal{H}}_{\beta}^{n}(1)\right| \int_{0}^{1} \int_{|z|>2}|z| \mu^{(n)}(\mathrm{d} t, \mathrm{~d} z)\right]+K \mathbb{E}\left[\left|\mathcal{R}_{1, \beta}^{n}(1)\right| \frac{1}{n^{1 / \alpha}} \int_{0}^{1} \int_{|z|>2}|z| \mu^{(n)}(\mathrm{d} t, \mathrm{~d} z)\right]
$$

From the proof of Lemma 5.4 in [6], we can deduce that $\left|\widehat{\mathcal{H}}_{\beta}^{n}(1) \int_{0}^{1} \int_{|z|>2}\right| z\left|\mu^{(n)}(\mathrm{d} t, \mathrm{~d} z)\right|$ is bounded by a random variable independent of $n$ and belonging to $\cap_{p \geq 1} \mathbf{L}^{p}$.

Using Cauchy-Schwarz inequality and (6.15), we get

$$
\mathbb{E}\left[\left|\mathcal{R}_{1, \beta}^{n}(1)\right| \frac{1}{n^{1 / \alpha}} \int_{0}^{1} \int_{|z|>2}|z| \mu^{(n)}(\mathrm{d} t, \mathrm{~d} z)\right] \leq \frac{C}{\sqrt{n}}\left(\mathbb{E}\left[\int_{0}^{1} \int_{|z|>2} \frac{|z|}{n^{1 / \alpha}} \mu^{(n)}(\mathrm{d} t, \mathrm{~d} z)\right]^{2}\right)^{1 / 2}
$$

Now from $\mu^{(n)}(\mathrm{d} s, \mathrm{~d} z)=\tilde{\mu}^{(n)}(\mathrm{d} s, \mathrm{~d} z)+v^{(n)}(\mathrm{d} s, \mathrm{~d} z)$, by convexity inequality, we have

$$
\begin{aligned}
& \mathbb{E}\left[\int_{0}^{1} \int_{|z|>2} \frac{|z|}{n^{1 / \alpha}} \mu^{(n)}(\mathrm{d} s, \mathrm{~d} z)\right]^{2} \leq 2 \mathbb{E}\left[\int_{0}^{1} \int_{|z|>2} \frac{|z|}{n^{1 / \alpha}} \tilde{\mu}^{(n)}(\mathrm{d} s, \mathrm{~d} z)\right]^{2}+2\left[\int_{0}^{1} \int_{|z|>2} \frac{|z|}{n^{1 / \alpha}} v^{(n)}(\mathrm{d} s, \mathrm{~d} z)\right]^{2} \\
& \leq 2 \mathbb{E}\left[\int_{0}^{1} \int_{2<|z| \leq 2 n^{1 / \alpha}} \frac{|z|^{2}}{n^{2 / \alpha}} \frac{1}{|z|^{1+\alpha}} \mathrm{d} z \mathrm{~d} s\right]+2\left[\int_{0}^{1} \int_{2<|z| \leq 2 n^{1 / \alpha}} \frac{|z|}{n^{1 / \alpha}} \frac{1}{|z|^{1+\alpha}} \mathrm{d} z \mathrm{~d} s\right]^{2} \\
& \leq \frac{C}{n^{2 / \alpha}}\left(\frac{n^{2 / \alpha}}{n}+\frac{n^{1 / \alpha}}{n}\right) \quad \text { where } C \text { is a deterministic constant. }
\end{aligned}
$$

From (6.56) and (6.57), we deduce that the left-hand side of (6.56) is bounded by $\frac{C}{n}$. Then we get that $\sup _{n} I_{1.3}^{n}<+\infty$.

For $I_{1.4}^{n}$, from the boundedness of $\mathcal{H}^{n, K},(6.21)$, and the fact that

$\Gamma\left(L_{1}^{n}, \mathcal{H}^{n, K}\right) \leq \Gamma\left(\mathcal{H}^{n, K}, \mathcal{H}^{n, K}\right)^{1 / 2} \Gamma\left(L_{1}^{n}, L_{1}^{n}\right)^{1 / 2}$ we have:

$$
\begin{aligned}
I_{1.4}^{n} & \leq \mathbb{E}\left[\left(\left|\frac{\Gamma\left(\mathcal{H}^{n, K}, \mathcal{H}^{n, K}\right)}{\Gamma\left(L_{1}^{n}, L_{1}^{n}\right)}\right|\right)^{1 / 2} \int_{0}^{1} \int_{|z|>2}|z| \mu^{(n)}(\mathrm{d} t, \mathrm{~d} z)\right] \\
& =\mathbb{E}\left[\frac{\left|\Gamma\left(\mathcal{H}^{n, K}, \mathcal{H}^{n, K}\right)\right|^{1 / 2}}{\left|\int_{0}^{1} \int_{\mathbb{R}} \rho^{n}(z) \mu^{(n)}(\mathrm{d} s, \mathrm{~d} z)\right|^{1 / 2}} \int_{0}^{1} \int_{|z|>2}|z| \mu^{(n)}(\mathrm{d} t, \mathrm{~d} z)\right] \\
& \leq \mathbb{E}\left[\frac{\left|\Gamma\left(\mathcal{H}^{n, K}, \mathcal{H}^{n, K}\right)\right|^{1 / 2}}{\left|\int_{0}^{1} \int_{|z|>2} z^{2} \mu^{(n)}(\mathrm{d} s, \mathrm{~d} z)\right|^{1 / 2}} \int_{0}^{1} \int_{|z|>2}|z| \mu^{(n)}(\mathrm{d} t, \mathrm{~d} z)\right] .
\end{aligned}
$$


Applying the Cauchy-Schwarz inequality, we have

$$
\int_{0}^{1} \int_{|z|>2} \mu^{n}(\mathrm{~d} t, \mathrm{~d} z) \times \int_{0}^{1} \int_{|z|>2} z^{2} \mu^{(n)}(\mathrm{d} s, \mathrm{~d} z) \geq\left(\int_{0}^{1} \int_{|z|>2}|z| \mu^{n}(\mathrm{~d} t, \mathrm{~d} z)\right)^{2} .
$$

We deduce

$$
\begin{aligned}
& \mathbb{E}\left[\frac{\left|\Gamma\left(\mathcal{H}^{n, K}, \mathcal{H}^{n, K}\right)\right|^{1 / 2}}{\left|\int_{0}^{1} \int_{|z|>2} z^{2} \mu^{(n)}(\mathrm{d} s, \mathrm{~d} z)\right|^{1 / 2}} \int_{0}^{1} \int_{|z|>2}|z| \mu^{(n)}(\mathrm{d} t, \mathrm{~d} z)\right] \\
& \leq \mathbb{E}\left[\left|\Gamma\left(\mathcal{H}^{n, K}, \mathcal{H}^{n, K}\right)\right|^{1 / 2}\left\{\int_{0}^{1} \int_{|z|>2} \mu^{(n)}(\mathrm{d} t, \mathrm{~d} z)\right\}^{1 / 2}\right] \\
& \leq\left\{\mathbb{E}\left[\left|\Gamma\left(\mathcal{H}^{n, K}, \mathcal{H}^{n, K}\right)\right|\right]\right\}^{1 / 2}\left\{\mathbb{E}\left[\int_{0}^{1} \int_{|z|>2} \mu^{(n)}(\mathrm{d} t, \mathrm{~d} z)\right]\right\}^{1 / 2} .
\end{aligned}
$$

From $\left\{\mathbb{E}\left[\int_{0}^{1} \int_{|z|>2} \mu^{(n)}(d t, d z)\right]\right\}^{1 / 2} \leq C_{4}$ where $C_{4}$ is a constant and the fact that $\Gamma\left(\mathcal{H}^{n, K}, \mathcal{H}^{n, K}\right)$ admits finite moment, independently of $n$ (but depending of $K$ ) then, (6.59) is bounded independently of $n$ and $I_{1.4}^{n}$ is proved. Hence (6.54) follows.

Step 2: We now prove $\sup _{n} n I_{2}^{n}<\mathrm{C}^{*}$ where $\mathrm{C}^{*}$ is a positive constant.

We have

$$
\begin{aligned}
\left|\frac{\Gamma\left(\frac{\sigma L_{1}^{n}}{n^{1 / \alpha}}, \bar{Y}_{1}^{n, \beta, x_{0}}-\varsigma_{1}^{n, \theta, x_{0}}\right)}{\Gamma\left(\frac{\sigma L_{1}^{n}}{n^{1 / \alpha}}, \frac{\sigma L_{1}^{n}}{n^{1 / \alpha}}\right)}-1\right| & =\frac{1}{n}\left|\int_{0}^{1} \frac{\Gamma\left(\frac{\sigma L_{1}^{n}}{n^{1 / \alpha}}, b\left(\bar{Y}_{s}^{n, \beta, x_{0}}, \theta\right)-b\left(\varsigma_{s}^{n, \theta, x_{0}}, \theta\right)\right)}{\Gamma\left(\frac{\sigma L_{1}^{n}}{n^{1 / \alpha}}, \frac{\sigma L_{1}^{n}}{n^{1 / \alpha}}\right)} \mathrm{d} s\right| \\
& \leq \frac{1}{n} \frac{\left\|b^{\prime}\right\|_{\infty}}{\Gamma\left(\frac{\sigma L_{1}^{n}}{n^{1 / \alpha}}, \frac{\sigma L_{1}^{n}}{n^{1 / \alpha}}\right)} \int_{0}^{1}\left|\Gamma\left(\frac{\sigma L_{1}^{n}}{n^{1 / \alpha}}, \bar{Y}_{s}^{n, \beta, x_{0}}\right)\right| \mathrm{d} s .
\end{aligned}
$$

Using that

$$
\left\{\begin{array}{l}
\Gamma\left(\frac{\sigma L_{1}^{n}}{n^{1 / \alpha}}, \bar{Y}_{s}^{n, \beta, x_{0}}\right) \leq \Gamma\left(\frac{\sigma L_{1}^{n}}{n^{1 / \alpha}}, \frac{\sigma L_{1}^{n}}{n^{1 / \alpha}}\right)^{1 / 2} \Gamma\left(\bar{Y}_{s}^{n, \beta, x_{0}}, \bar{Y}_{s}^{n, \beta, x_{0}}\right)^{1 / 2} \\
\Gamma\left(\bar{Y}_{s}^{n, \beta, x_{0}}, \bar{Y}_{s}^{n, \beta, x_{0}}\right)^{1 / 2} \leq M \Gamma\left(\bar{Y}_{1}^{n, \beta, x_{0}}, \bar{Y}_{1}^{n, \beta, x_{0}}\right)^{1 / 2} \\
\Gamma\left(\bar{Y}_{1}^{n, \beta, x_{0}}, \bar{Y}_{1}^{n, \beta, x_{0}}\right)^{1 / 2} \leq M_{1} \Gamma\left(\frac{\sigma L_{1}^{n}}{n^{1 / \alpha}}, \frac{\sigma L_{1}^{n}}{n^{1 / \alpha}}\right)^{1 / 2}
\end{array}\right.
$$

for some constants $M$ and $M_{1}$, we can easily deduce that

$$
\left|\frac{\Gamma\left(\frac{\sigma L_{1}^{n}}{n^{1 / \alpha}}, \bar{Y}_{1}^{n, \beta, x_{0}}-\varsigma_{1}^{n, \theta, x_{0}}\right)}{\Gamma\left(\frac{\sigma L_{1}^{n}}{n^{1 / \alpha}}, \frac{\sigma L_{1}^{n}}{n^{1 / \alpha}}\right)}-1\right| \leq \frac{C^{*}}{n}
$$

for a positive constant $C^{*}$. 
Lemma 6.11. Under the assumptions of Proposition 6.8, for any compact set $Q \subset \mathbb{R} \times(0, \infty), \forall K>2$, there exists a constant $c_{K}>0$ such that

$$
\sup _{x_{0}, \beta \in Q}\left\|\eta^{\mathcal{H}^{n, K}}\left(\bar{Y}_{1}^{n, \beta, x_{0}}-\varsigma_{1}^{n, \theta, x_{0}}\right)-\bar{\eta}^{\mathcal{H}^{n, K}}\left(\bar{Y}_{1}^{n, \beta, x_{0}}-\varsigma_{1}^{n, \theta, x_{0}}\right)\right\|_{1} \leq \frac{c_{K}}{n} .
$$

Proof. We estimate the $\mathbf{L}^{1}$-norm appearing in (6.60) by duality. Let $\bar{\beta}: \mathbb{R} \rightarrow[-1,1]$ be a measurable function, we evaluate:

$$
\begin{aligned}
& \left|\mathbb{E}\left[\left(\eta^{\mathcal{H}^{n, K}}\left(\bar{Y}_{1}^{n, \beta, x_{0}}-\varsigma_{1}^{n, \theta, x_{0}}\right)-\bar{\eta}^{\mathcal{H}^{n, K}}\left(\bar{Y}_{1}^{n, \beta, x_{0}}-\varsigma_{1}^{n, \theta, x_{0}}\right)\right) \bar{\beta}\left(\bar{Y}_{1}^{n, \beta, x_{0}}-\varsigma_{1}^{n, \theta, x_{0}}\right)\right]\right| \\
& \leq\left|\mathbb{E}\left[\eta^{\mathcal{H}^{n, K}}\left(\bar{Y}_{1}^{n, \beta, x_{0}}-\varsigma_{1}^{n, \theta, x_{0}}\right) \bar{\beta}\left(\bar{Y}_{1}^{n, \beta, x_{0}}-\varsigma_{1}^{n, \theta, x_{0}}\right)\right]-\mathbb{E}\left[\bar{\eta}^{\mathcal{H}^{n, K}}\left(\frac{\sigma L_{1}^{\alpha}}{n^{1 / \alpha}}\right) \bar{\beta}\left(\frac{\sigma L_{1}^{\alpha}}{n^{1 / \alpha}}\right)\right]\right| \\
& +\left|\mathbb{E}\left[\bar{\eta}^{\mathcal{H}^{n, K}}\left(\frac{\sigma L_{1}^{\alpha}}{n^{1 / \alpha}}\right) \bar{\beta}\left(\frac{\sigma L_{1}^{\alpha}}{n^{1 / \alpha}}\right)\right]-\mathbb{E}\left[\bar{\eta}^{\mathcal{H}^{n, K}}\left(\bar{Y}_{1}^{n, \beta, x_{0}}-\varsigma_{1}^{n, \theta, x_{0}}\right) \bar{\beta}\left(\bar{Y}_{1}^{n, \beta, x_{0}}-\varsigma_{1}^{n, \theta, x_{0}}\right)\right]\right| \\
& \leq\left|\mathbb{E}\left[\eta^{\mathcal{H}^{n, K}}\left(\bar{Y}_{1}^{n, \beta, x_{0}}-\varsigma_{1}^{n, \theta, x_{0}}\right) \bar{\beta}\left(\bar{Y}_{1}^{n, \beta, x_{0}}-\varsigma_{1}^{n, \theta, x_{0}}\right)\right]-\mathbb{E}\left[\bar{\eta}^{\mathcal{H}^{n, K}}\left(\frac{\sigma L_{1}^{\alpha}}{n^{1 / \alpha}}\right) \bar{\beta}\left(\frac{\sigma L_{1}^{\alpha}}{n^{1 / \alpha}}\right)\right]\right|+\frac{C_{K}}{n} K
\end{aligned}
$$

where we have used Lemma 6.10 with the choice $\mathcal{H}^{n}=1, K>2$ and the choice $h=\bar{\eta}^{\mathcal{H}^{n, K}} \bar{\beta}$, recalling that $\left\|\bar{\eta}^{\mathcal{H}^{n, K}}\right\|_{\infty} \leq K$. From the definition of $\bar{\eta}^{\mathcal{H}^{n, K}}\left(\frac{\sigma L_{1}^{\alpha}}{n^{1 / \alpha}}\right)$ and $\eta^{\mathcal{H}^{n, K}}\left(\bar{Y}_{1}^{n, \beta, x_{0}}-\varsigma_{1}^{n, \theta, x_{0}}\right)$ as conditional expectations, we have:

$$
\begin{aligned}
& \left|\mathbb{E}\left[\eta^{\mathcal{H}^{n, K}}\left(\bar{Y}_{1}^{n, \beta, x_{0}}-\varsigma_{1}^{n, \theta, x_{0}}\right) \bar{\beta}\left(\bar{Y}_{1}^{n, \beta, x_{0}}-\varsigma_{1}^{n, \theta, x_{0}}\right)\right]-\mathbb{E}\left[\bar{\eta}^{\mathcal{H}^{n, K}}\left(\frac{\sigma L_{1}^{\alpha}}{n^{1 / \alpha}}\right) \bar{\beta}\left(\frac{\sigma L_{1}^{\alpha}}{n^{1 / \alpha}}\right)\right]\right| \\
& =\left|\mathbb{E}\left[\mathcal{H}^{n} \mathcal{X}_{K}\left(\mathcal{H}^{n}\right) \bar{\beta}\left(\bar{Y}_{1}^{n, \beta, x_{0}}-\varsigma_{1}^{n, \theta, x_{0}}\right)\right]-\mathbb{E}\left[\mathcal{H}^{n} \mathcal{X}_{K}\left(\mathcal{H}^{n}\right) \bar{\beta}\left(\frac{L_{1}^{\alpha}}{n^{1 / \alpha}}\right)\right]\right| \leq \frac{C_{K}}{n},
\end{aligned}
$$

where we used Lemma 6.10. This gives,

$$
\sup _{\|\bar{\beta}\|_{\infty} \leq 1}\left|\mathbb{E}\left[\left(\eta^{\mathcal{H}^{n, K}}\left(\bar{Y}_{1}^{n, \beta, x_{0}}-\varsigma_{1}^{n, \theta, x_{0}}\right)-\bar{\eta}^{\mathcal{H}^{n, K}}\left(\bar{Y}_{1}^{n, \beta, x_{0}}-\varsigma_{1}^{n, \theta, x_{0}}\right)\right) \bar{\beta}\left(\bar{Y}_{1}^{n, \beta, x_{0}}-\varsigma_{1}^{n, \theta, x_{0}}\right)\right]\right| \leq(1+K) \frac{C_{K}}{n}
$$

and we deduce the result of this lemma.

\section{REFERENCES}

[1] Y. Aït-Sahalia and J. Jacod, Volatility estimators for discretely sampled Lévy processes. Ann. Stat. 35 (2007) 355-392.

[2] Y. Aït-Sahalia and J. Jacod, Fisher's information for discretely sampled Lévy processes. Econometrica 76 (2008) $727-761$.

[3] O.E. Barndorff-Nielsen, T. Mikosch and S.I. Resnick (Eds.), Theory and applications, Lévy Processes. Birkhäuser Boston, Inc., Boston, MA (2001).

[4] K. Bichteler, J.-B. Gravereaux and J. Jacod, Malliavin Calculus for Processes with Jumps. Vol. 2 of Stochastics Monographs. Gordon and Breach Science Publishers, New York (1987).

[5] E. Clément and A. Gloter, Local asymptotic mixed normality property for discretely observed stochastic differential equations driven by stable Lévy processes. Stoch. Process. Appl. 125 (2015) 2316-2352.

[6] E. Clément, A. Gloter and H. Nguyen, Asymptotics in Small Time for the Density of a Stochastic Differential Equation Driven by a Stable Lévy Process. Preprint HAL-01410989v2 (2017).

[7] V. Genon-Catalot and J. Jacod, On the estimation of the diffusion coefficient for multi-dimensional diffusion processes. Ann. Inst. Henri Poincaré Probab. Stat. 29 (1993) 119-151.

[8] E. Gobet, Local asymptotic mixed normality property for elliptic diffusion: a Malliavin calculus approach. Bernoulli 7 (2001) 899-912.

[9] E. Gobet, LAN property for ergodic diffusions with discrete observations. Ann. Inst. Henri Poincaré Probab. Stat. 38 (2002) $711-737$. 
[10] J. Hájek, A characterization of limiting distributions of regular estimates. Z. Wahrscheinlichkeitstheorie Verw. Gebiete 14 (1969/1970) 323-330.

[11] P. Hall and C.C. Heyde, Martingale Limit Theory and its Application. Probability and Mathematical Statistics. Academic Press, Inc. [Harcourt Brace Jovanovich, Publishers], New York, London (1980).

[12] D. Ivanenko, A.M. Kulik and H. Masuda, Uniform LAN property of locally stable Lévy process observed at high frequency. ALEA Lat. Am. J. Probab. Math. Stat. 12 (2015) 835-862.

[13] J. Jacod, The Euler scheme for Lévy driven stochastic differential equations: limit theorems. Ann. Probab. 32 (2004) $1830-1872$.

[14] P. Jeganathan, On the asymptotic theory of estimation when the limit of the log-likelihood ratios is mixed normal. Sankhya Ser. A 44 (1982) 173-212.

[15] P. Jeganathan, Some asymptotic properties of risk functions when the limit of the experiment is mixed normal. Sankhya Ser. A 45 (1983) 66-87.

[16] B.-Y. Jing, X.-B. Kong and Z. Liu, Modeling high-frequency financial data by pure jump processes. Ann. Stat. 40 (2012) 759-784.

[17] R. Kawai and H. Masuda, On the local asymptotic behavior of the likelihood function for Meixner Lévy processes under high-frequency sampling. Stat. Probab. Lett. 81 (2011) 460-469.

[18] R. Kawai and H. Masuda, Local asymptotic normality for normal inverse Gaussian Lévy processes with high-frequency sampling. ESAIM: PS $\mathbf{1 7}$ (2013) 13-32.

[19] V.N. Kolokoltsov, Markov Processes, Semigroups and Generators. Vol. 38 of de Gruyter Studies in Mathematics. Walter de Gruyter \& Co., Berlin (2011).

[20] X.-B. Kong, Z. Liu and B.-Y. Jing, Testing for pure-jump processes for high-frequency data. Ann. Stat. 43 (2015) $847-877$.

[21] L. Le Cam and G.L. Yang, Asymptotics in Statistics: Some Basic Concepts. Springer Series in Statistics. Springer-Verlag, New York (1990).

[22] H. Masuda, Joint estimation of discretely observed stable Lévy processes with symmetric Lévy density. J. Jpn. Stat. Soc. 39 (2009) 49-75.

[23] H. Masuda, Non-Gaussian Quasi-Likelihood Estimation of sde Driven by Locally Stable Lévy Process. Preprint arXiv:1608.06758v3 (2017).

[24] J. Picard, On the existence of smooth densities for jump processes. Probab. Theory Relat. Fields 105 (1996) $481-511$. 